\title{
Towards incorporating anamorphic fungi in a natural classification - checklist and notes for 2011
}

\author{
Wijayawardene DNN ${ }^{1,2}$, McKenzie $\mathrm{EHC}^{3}$ and Hyde $\mathrm{KD}^{1,2}$ \\ ${ }^{1}$ Institute of Excellence in Fungal Research, Mae Fah Luang University, Chiang Rai 57100, Thailand \\ ${ }^{2}$ School of Science, Mae Fah Luang University, Chiang Rai 57100, Thailand \\ ${ }^{3}$ Manaaki Whenua Landcare Research, Private Bag 92170, Auckland, New Zealand
}

Wijayawardene DNN, McKenzie EHC, Hyde KD 2012 - Towards incorporating anamorphic fungi in a natural classification - checklist and notes for 2011. Mycosphere 3(2), 157-228, Doi 10.5943 /mycosphere/3/2/5

A complilation of anamorphic names for both Ascomycota and Basidiomycota is provided which comprises 2895 genera. The genera are listed against a backbone of teleomorphic relationships where known. The study reveals that 73 genera and 95 anamorph-like genera are linked to teleomorphic genera names, 447 genera (three anamorph-like genera) are linked to teleomorph families, orders or classes, while for more than 1592 (55\%) genera no teleomorph link is known. The links are based on the literature and often because fungi were found in association, but have not been proven by molecular data. Many are based on links with species other than the generic type and thus must be considered questionable for the genus. A considerable effort is needed to establish whether these links are correct.

Key words - Asexual fungi - life cycle - sexual fungi - taxonomy

\author{
Article Information \\ Received 24 March 2012 \\ Accepted 29 March 2012 \\ Published online 10 April 2012 \\ Corresponding author: KD Hyde - e-mail - kdhyde3@gmail.com
}

\section{Introduction}

The purpose of the present paper is to collate the knowledge of asexual fungi in relationship to their sexual states. It attempts to classify the asexual genera in a natural biological framework for the Ascomycota based on the Outline of Ascomycota 2009 (Lumbsch \& Huhndorf 2010) and for the Basidiomycota based on Kirk et al. (2008). The present paper has used data from the previous outline of Hyde et al. (2010) as well as literature published in 2011 and updated with opinions in Seifert et al. (2011).

There is now a mandate in the systematics community to place one name on a holomorphic fungus species as opposed to the previous system (especially in Ascomycota) of naming both sexual and asexual morphs
(Samuels et al. 2009, Hawksworth 2011; Hawksworth et al. 2011, Taylor 2011; Wingfield et al. 2011). Also, as more and more strains of asexual genera are sequenced (Shenoy et al. 2010, Põldmaa 2011, Reblova \& Seifert 2011), these genera will be classified in families, orders and classes of Ascomycota and Basidiomycota. This will result in more and more asexual states being described and illustrated without being given formal names and therefore it is important to have a forum where asexual data can be brought to the attention of researchers. It is hoped that this compilation will facilitate the move towards one fungus one name (Shenoy et al. 2007, 2010, Hawksworth et al. 2011). By placing all asexual genera against the backbone classification of the sexual genera it will be possible to establish, 1) 
what are the sexual states of various species, genera,families or orders, 2) which genera have no information, 3 ) which are the older or more commonly used names for each genus, and 4) illustrate the redundancy of the dual classification system.

This compilation also provides a single place where asexual connections can be accessed and critically scrutinized so that a stable and reliable system can be developed based on published data. We do not claim that this compilation is complete or definitive; however, it provides a forum for displaying data and can be added to or changed as more data becomes available or errors are discovered. In future issues we will explore various connections and provide more data.

In the long term it will be desirable if Myconet (http://www.fieldmuseum.org/myconet) is merged with this document, however, this should occur at some time in the future, when more data is available.

\section{Material and methods}

The overall scheme for the classification of the sexual fungi follows the Outline of Ascomycota 2009 (Lumbsch \& Huhndorf 2010), with additional data on anamorphic Basidiomycota from Kirk et al. (2008). This scheme is chosen as the schemes in the Dictionary of the Fungi, Index Fungorum, and MycoBank differ to a greater or lesser extent and it was felt wise to follow what the authors consider is an admirable attempt to keep abreast of the current literarure as is routinely carried out in the Outline of Ascomycota. This compilation also annotates changes made in the form of notes on asexual fungi (marked with an asterisk after the entry, with notes in the later "Notes" section) so that changes can easily be followed; this is not always true of the available databases.

Asexual genera or '-like' genera are listed in bold under the appropriate fungal classification and the link to a teleomorph genus given where found. Dubious genera are excluded but can be added if they are later found to be good genera. Fossil fungi are also excluded as it would be impossible with present techniques available to provide a natural classification for them. A question mark before the entry means that its placement in that family or order is not fully established and generally follows Lumbsch \& Huhndorf (2010). A question mark before anamorph means it is not clear that this is the anamorph of this genus, although there are many cases without a question mark where the link has not equivocally been proven. An asterisk following the names indicates an entry in the "Notes" section.

In many genus records in Index Fungorum the species is linked to more than one anamorphic genus. Such records can be located by searching the genus throughout the list for repeat entries. The synonyms of genera are not listed as they can be easily found in Index Fungorum.

We encourage colleagues to inform us of any missing entries, new data or misunderstandings, which will be updated in future compilations. We would also invite experts of specific groups to contribute to future compilations on their specific group(s) of interest and encourage reviews or illustrated accounts of families, orders or other groups relating to sexual and asexual links. Reviews of asexual genera with sections or series, such as Aspergillus, Penicillium and Verticillium, the powdery mildews, many of the yeasts and yeast-like genera and the asexual smuts and rusts are particularly needed. Similarly the lichenized ascomycete asexual states are poorly documented.

As there is mandate towards using one name for both the sexual and asexual states of a fungus we also encourage authors to submit articles or opinions concerning the use of either name. This might be in the form of a general critic, arguments for or against using one name, or an article that specifically addresses an individual group or genus.

In the present outline we have added $(\mathrm{H})=$ hyphomycete,$(\mathrm{C})=$ coelomycete,$(\mathrm{Y})=$ yeast and $(\mathrm{R})=$ Rust against entries where known. Although this grouping is artificial we do this to facilitate finding data on either as the groups are generally dealt with in different text. For example, data for the hyphomycetes have recently been complied and updated by Seifert et al. (2011) and the new data for hyphomycetes included here have been extracted from this tome. Data for coelomycetes were extracted from Sutton (1980), Nag Raj (1993), http:// 
Mycosphere Doi 10.5943/mycosphere/3/2/5

www. Cybertr uffle.org.uk/cybernome/eng/index.htm (Accesion date 18.03.2011) and http://www.landcarer esearch .co.nz/html /data asp? ID $=$ \&NAMEKey $=26885$ (Accesion date 6.12.2011).

\section{Index to Ascomycota}

Phylum ASCOMYCOTA Caval-Sm.

Subphylum TAPHRINOMYCOTINA O.E. Erikss. \& Winka

Class Taphrinomycetes O.E. Erikss. \& Winka

Taphrinales Gäum. \& C.W. Dodge

Protomycetaceae Gray

Saitoella Goto, Sugiy., Hamam. \& Komag. 1987 (Y)

Taphrinaceae Gäum.

Lalaria R.T. Moore 1990, anamorphic Taphrina Fr. 1815 (Y)

Subphylum SACHAROMYCOTINA O.E. Erikss. \& Winka

Class Saccharomycetes O.E. Erikss. \& Winka Saccharomycetales Kudrjanzev

Dipodascaceae Engl. \& E. Gilg

Blastobotrys Klopotek 1967, anamorphic Trichomonascus H.S. Jacks. 1948 (H)

?Candida Berkhout 1923, anamorphic Sporopachydermia Rodr. Mir. 1978 (Y)

Geotrichum Link 1809, anamorphic Galactomyces Redhead \& Malloch 1977 and Dipodascus Lagerh. 1892 (H)

Geotrichum-like, anamorphic Basidioascus Matsush. 2003 (H)

Saprochaete Coker \& Shanor 1939, anamorphic Magnusiomyces Zender 1926 (H)

Endomycetaceae J. Schröt.

Fusidium Link 1809, possibly anamorphic Ascocephalophora K. Matsush. \& Matsush. 1995 (H)

Lipomycetaceae E.K. Novák \& Zsolt

Myxozyma Van der Walt, Weijman \& Arx $1981(\mathbf{Y})$

Metschnikowiaceae T. Kamienski

Candida Berkhout 1923, anamorphic Clavispora Rodr. Mir. 1979 and
Metschnikowia T. Kamienski 1899 (Y)

Pichiaceae Zender

Brettanomyces N.H. Claussen ex Custers 1940, anamorphic Dekkera Van der Walt 1964 (Y)

Eeniella M.T. Sm., Bat. Vegte \& Scheffers 1981, anamorphic Dekkera Van der Walt (Y)

Enantiothamnus Pinoy 1911 (Y)

Hyphopichia Arx \& Van der Walt 1976 (Y)

Saccharomycetaceae G. Winter

?Candida Berkhout 1923, anamorphic Ogataea Y. Yamada, K. Maeda \& Mikata 1994, Lodderomyces Van der Walt 1966 and Spathaspora N.H. Nguyen, S.O. Suh \& M. Blackw. 2006 (Y)

Saccharomycodaceae Kudrjanzev

Candida Berkhout 1923, anamorphic Lodderomyces Van der Walt 1966 and Spathaspora N.H. Nguyen, S.O. Suh \& M. Blackw. 2006 (Y)

Kloeckera Janke 1923, anamorphic Hanseniaspora Zikes 1911 (Y)

Trichomonascaceae Kurtzman \& Robnett

?Candida Berkhout 1923, anamorphic Zygoascus M.T. Sm. 1986 (Y)

Saccharomycetales, genera incertae sedis

Aciculoconidium D.S. King \& S.C. Jong 1976 (Y)

Botryozyma Shann \& M.T. Sm. 1992, anamorphic Ascobotryozyma J. Kerrigan, M.T. Sm. \& J.D. Rogers 2001 (Y)

Candida Berkhout 1923, anamorphic Starmerella C.A. Rosa \& Lachance 1998 (Y)

Cicadomyces Šulc 1911 (Y)

Macrorhabdus Tomasz., Logan, Snowden, Kurtzman \& Phalen 2003 (Y)

Oosporidium Stautz 1931 (Y)

Pseudomycoderma H. Will 1916 (Y)

Schizoblastosporion Cif. 1930 (Y)

Trigonopsis Schachner 1929 (Y)

Saccharomycetes, genera incertae sedis

Selenotila Lagerh. 1892 (Y) 
Selenozyma Yarrow 1977 (Y)

Subphylum PEZIZOMYCOTINA O.E. Erikss. \& Winka

Class Arthoniomycetes O.E. Erikss. \& Winka

Arthoniales Henssen ex D. Hawksw. \& O.E. Erikss.

Arthoniaceae Reichenb. ex Reichenb.

Helicobolomyces Matzer 1995, anamorphic Arthonia Ach. 1806 (C)

?Septocyta Petr. 1927, anamorphic Arthonia Ach. 1806 (C)

Subhysteropycnis Wedin \& Hafellner 1998, anamorphic Arthonia Ach. 1806 (C)

Roccellaceae Chevall.

Sporhaplus H.B.P. Upadhyay 1964, anamorphic Mazosia A. Massal 1854 (C)

Class Dothideomycetes sensu O.E. Erikss. \& Winka

Subclass Dothideomycetidae P.M. Kirk, P.F. Cannon, J.C. David \& J.A. Stalpers ex C.L. Schoch, Spatafora, Crous \& Shoemaker

Capnodiales Woron.

Antennulariellaceae Woron.

Capnodendron S. Hughes 1976, anamorphic Antennulariella Woron. 1915 (H)

Capnofrasera S. Hughes 2003 (H)

Heteroconium Petr. 1949, anamorphic Antennulariella Woron. 1915 (H)

Capnodiaceae (Sacc.) Höhn. ex Theiss.

Acanthorus Bat. \& Cavalc. 1967 (C)

?Antennariella Bat. \& Cif. 1963 (C)*

Apiosporium Kunze 1817 (C)

Conidiocarpus Woron. 1927, anamorphic Phragmocapnias Theiss. \& Syd. 1918 and Scorias Fr. 1825 (C)*

Fumagospora G. Arnaud 1911, anamorphic Capnodium Mont. 1848 (C)

?Fumiglobus D.R. Reynolds \& G.S. Gilbert 2006 (C)*

Microxiphium (Harv. ex Berk. \& Desm.) Thüm. 1879 (as Microxyphium) $(\mathbf{H})^{*}$

?Mycogelidium W.Y. Zhuang 2007 (C)

Phaeoxyphiella Bat. \& Cif. 1963, anamorphic Capnodium Mont. 1848 (C)*

?Polychaetella Speg. 1918, anamorphic Capnodium Mont. 1848 (C)*

Polychaeton (Pers.) Lev. 1846 (C)*
Mycosphere Doi 10.5943/mycosphere/3/2/5

Scolecoxyphium Cif. \& Bat. 1956, anamorphic Capnodium Mont. 1848 and Scorias Fr. 1825 (C)*

Tripospermum Speg. 1918 anamorphic Triposporiopsis W.Yamam 1955 (H)*

Coccodiniaceae Höhn. ex O.E. Erikss.

Bisbyopeltis Bat. \& A.F. Vital 1957 (C)

Microxiphium (Harv. ex Berk. \& Desm.) Thüm. 1879, anamorphic Dennisiella Bat. \& Cif. 1962 (H)*

Davidiellaceae C.L. Schoch, Spatafora, Crous \& Shoemaker

Acroconidiella J.C. Lindq. \& Alippi 1964, anamorphic Davidiella Crous \& U. Braun 2003 (H)

Cladosporium Link 1816, anamorphic Davidiella Crous \& U. Braun 2003 (H)

Dichocladosporium K. Schub., U. Braun \& Crous 2007 (C)

Graphiopsis Trail 1889, anamorphic Davidiella Crous \& U. Braun 2003 (H)

Hoornsmania Crous 2007 (H)

Rachicladosporium Crous, U. Braun \& C.F. Hill 2007 (H)

Toxicocladosporium Crous \& U. Braun 2007 (H)

Verrucocladosporium K. Schub., Aptroot \& Crous $2007(\mathbf{H})$

Dissoconiaceae Crous \& de Hoog

Dissoconium de Hoog, Oorschot \& Hijwegen 1983 (H)

Ramichloridium Stahel 1937 ex de Hoog 1977 (H)

Metacapnodiaceae S. Hughes \& Corlett

Capnobotrys S. Hughes 1970, anamorphic Metacapnodium Speg. 1918 (H)

Capnocybe S. Hughes 1966, anamorphic Metacapnodium Speg. 1918 (H)

Capnophialophora S. Hughes 1966, anamorphic Metacapnodium Speg. 1918 (H)

Capnosporium S. Hughes 1976, anamorphic Metacapnodium Speg. 1918 (H)

Hormiokrypsis Bat. \& Nascim. 1957, anamorphic Metacapnodium Speg. 1918 and Ophiocapnocoma Bat. \& Cif. 1963 (H)

Torulopsiella Bender 1932, anamorphic 
Ophiocapnocoma Bat. \& Cif. 1963 and Metacapnodium Speg. 1918 (H)

Mycosphaerellaceae Lindau

Anguillosporella U. Braun 1995 (H)

Asperisporium Maubl. 1913 (H)

Asteromella-like, anamorphic Gillotia Sacc. \& Trotter 1913 (C)

Cercospora Fresen. 1863, anamorphic Mycosphaerella Johanson 1884, Sphaerulina Sacc. and others $(\mathbf{H})$

Cercosporella Sacc. 1880, anamorphic Mycosphaerella Johanson 1884 (H)

?Clypeispora A.W. Ramaley 1991, anamorphic Mycosphaerella Johanson 1884 (C)

Colletogloeum Petr. 1953, anamorphic Mycosphaerella Johanson 1884 (C)

Coniothryium-like, anamorphic Bruneosphaerella Crous 2009 (C)

Deightoniella S. Hughes 1952 (H)

Didymochora Höhn. 1918, anamorphic Euryachora Fuckel 1870 (C)

Distocercospora N. Pons \& B. Sutton 1988 (H)

Dothistroma Hulbary 1941 (C)

Floricola Kohlm. \& Volkm.Kohlm. 2000 (C)

Fusicladiella Höhn. 1919, anamorphic Mycosphaerella Johanson 1884 (H)

Laocoön J.C. David 1997 (H)

Lecanosticta Syd. 1922 (C)

Microcyclospora Jana Frank, Schroers \& Crous 2010 (H)

Microcyclosporella Jana Frank, Schroers \& Crous $2010(\mathbf{H})$

Miuraea Hara 1948, anamorphic Mycosphaerella Johanson 1884 (H)

Mycopappus Redhead \& G.P. White 1985, anamorphic Mycosphaerella Johanson 1884 (H)

Mycovellosiella Rangel 1918, anamorphic Mycosphaerella Johanson 1884 (H)

Parastenella J.C. David 1991 (H)

Passalora Fr. 1849, anamorphic Mycosphaerella Johanson 1884 (H)

Penidiella Crous \& U. Braun 2007 (H)

Periconiella Sacc. 1885, anamorphic Allosoma Syd. 1926 (H)

Phacellium Bonord. 1860, anamorphic Mycosphaerella Johanson 1884 (H)

Phaeoisariopsis Ferraris 1909, anamorphic
Mycosphere Doi 10.5943/mycosphere/3/2/5

Mycosphaerella Johanson 1884 (H)

Phaeophleospora Rangel 1916 (C)

Phaeothecoidea Crous 2007 (H)

Phloeospora Wallr. 1833, anamorphic Mycosphaerella Johanson 1884 (C)

Placosphaeria-like, anamorphic Euryachora Fuckel 1870 (C)

Polythrincium Kunze 1817, anamorphic Cymadothea F.A. Wolf 1935 (H)

Pseudocercospora Speg. 1910 anamorphic Mycosphaerella Johanson 1884 (H)

Pseudocercosporella Deighton 1973, anamorphic Mycosphaerella Johanson 1884 (H)

Pseudocercosporidium Deighton 1973 (H)

Quasiphloeospora B. Sutton, Crous \& Shamoun 1996 (H)

Ramichloridium Stahel ex de Hoog 1977 (H)

Ramichloridium-like, anamorphic Mycosphaerella Johanson 1884 (H)

Ramularia Unger 1833, anamorphic Mycosphaerella Johanson 1884 and Melanodothis R.H. Arnold 1972 (H)

Ramulariopsis Speg. 1910, anamorphic Mycosphaerella Johanson 1884 (H)

Ramulispora Miura 1920, anamorphic Mycosphaerella Johanson 1884 (H)

Rhabdospora (Durieu \& Mont. ex Sacc.) Sacc. 1884 (C)

Rhexocercosporidium U. Braun 1994 (H)

Semipseudocercospora J.M. Yen 1983 (H)

Septoria Sacc. 1884 (C)

Septoria-like, anamorphic Mycosphaerella Johanson 1884 (C)

Sirosporium Bubák \& Serebrian. 1912 (H)

Sonderhenia H.J. Swart \& J. Walker 1988 (C)

Stenella Syd. 1930, anamorphic Mycosphaerella Johanson 1884 (H)

Stenellopsis B. Huguenin 1966 (H)

Stigmina Sacc. 1980, anamorphic Mycosphaerella Johanson 1884 (H)

Thedgonia B. Sutton 1973, anamorphic Mycosphaerella Johanson 1884 (H)

Thedgonia-like (H)

Trochophora R.T. Moore 1955 (H)

Verrucisporota D.E. Shaw \& Alcorn 1993 (H)

Xenostigmina Crous 1998, anamorphic Mycosphaerella Johanson 1884 or Didymella Sacc. ex D. Sacc. 1880 (H) 
Zasmidium Fr. 1849, anamorphic Mycosphaerella-like and Rasutoria M.E. Barr 1987 (H)

Zymoseptoria Quaedvlieg \& Crous 2011 (C)*

Capnodiales, genera incertae sedis

Capnocheirides J.L. Crane \& S. Hughes 1982 (H)

Heptaster Cif., Bat. \& Nascim. 1956 (H)

Hormonema-like, anamorphic Xenomeris Syd. 1924 (H)

Houjia G.Y. Sun \& Crous 2010 (H)

Micropustulomyces R.W. Barreto 1995 (C)

Phaeotheca Sigler, Tsuneda \& J.W. Carmich. 1981 (H)

Phaeothecoidiella Batzer \& Crous 2010 (H)

Pseudovirgaria H.D. Shin, U. Braun, Arzanlou \& Crous 2007 (H)

Rachicladosporium Crous, U. Braun \& C.F. Hill 2007 (H)

Recurvomyces Selbmann \& de Hoog 2008 (H)

Scolecostigmina U. Braun 1999 (H)

Sporidesmajora Batzer \& Crous 2010 (H)

Verrucocladosporium K. Schub., Aptroot \& Crous $2007(\mathbf{H})$

Dothideales Lindau

Dothideaceae Chevall.

Lecanosticta Syd. 1922, anamorphic Scirrhia Nitschke ex Fuckel 1870 (C)

Podoplaconema Petr. 1921, anamorphic Omphalospora Theiss. \& Syd. 1915 (C)

Dothioraceae Theiss. \& H. Syd.

Aureobasidium Viala \& G. Boyer 1891, anamorphic Columnosphaeria Munk 1953 and Saccothecium Fr. 1836 (H)

Dothichiza Lib. ex Roum. 1880, anamorphic Dothiora Fr. 1849 and Sydowia Bres. 1895 (C)

Hormonema Lagerb. \& Melin 1927, anamorphic Dothiora Fr. 1849, Pringsheimia Schulzer 1866 and Sydowia Bres. 1895 (H)

Japonia Höhn. 1909, anamorphic Yoshinagaia Henn. 1904 (C)

Kabatina R. Schneid. \& Arx 1966 (C)

?Rhizosphaera L. Mangin \& Har. 1907, anamorphic Phaeocryptopus Naumov
Mycosphere Doi 10.5943/mycosphere/3/2/5

1915 and Dimerina Theiss. 1912 (C)

Sclerophoma Höhn. 1909, anamorphic Sydowia Bres. 1895 (C)

Teratosphaeriaceae Crous \& U. Braun

Acidomyces B.J. Baker, M.A. Lutz, S.C. Dawson, P.L. Bond \& Banfield ex Selbmann, de Hoog \& De Leo 2008 (H)

Batcheloromyces Marasas, P.S. van Wyk \& Knox-Dav. 1975 (H)

Baudoinia J.A. Scott \& Unter. 2007 (H)

Capnobotryella Sugiy. 1987 (H)

Catenulostroma Crous \& U. Braun 2007, anamorphic Teratosphaeria Syd. \& P. Syd. 1912 (H)

Cibiessia Crous 2007 (H)

Colletogloeopsis Crous \& M.J. Wingf. 1997, anamorphic Teratosphaeria Syd. \& P. Syd. 1912 (C)

Davisoniella H.J. Swart 1988 (C)

Devriesia Seifert \& N.L. Nick. 2004 (H)

Elasticomyces Zucconi \& Selbmann 2008 (H)

Friedmanniomyces Onofri 1999 (H)

Hispidoconidioma Tsuneda \& Davey 2010 (H)

Hobsoniopsis D. Hawksw. 2001 (H)

Hortaea Nishim. \& Miyaji 1984 (H)

Kirramyces J. Walker, B. Sutton \& Pascoe 1992, anamorphic Teratosphaeria Syd. \& P. Syd. 1912 (C)

Nothostrasseria Nag Raj 1983 (C)

Passalora Fr. 1849 (H)

Penidiella Crous \& U. Braun 2007 (H)

Phacellium Bonord. 1860 (H)

Phaeothecoidea Crous 2007 (H)

Pseudocercosporella Deighton 1973 (H)

Pseudoramichloridium Cheewangkoon \& Crous 2010 (H)

Pseudotaeniolina J.L. Crane \& Schokn. 1986 (H)

Ramichloridium Stahel ex de Hoog 1977 (H)

Recurvomyces Selbmann \& de Hoog 2008 (H)

Readeriella Syd. \& P. Syd. 1908 (C)

Sporidesmium Link 1809 (H)

Staninwardia B. Sutton 1971 (C)

Stenella Syd. 1930 (H)

Trimmatostroma-like, anamorphic Teratosphaeria Syd. \& P. Syd. 1912 (H)

Tripospermum Speg. 1918 (H) 
Myriangiales Starbäck

Elsinoaceae Höhn. ex Sacc. \& Trotter

Endosporium Tsuneda 2008 (H)

Sphaceloma de Bary 1874 anamorphic Elsinö Racib. 1900 (C)

Xenodiella Syd. 1935, anamorphic Xenodium Syd. 1935 (H)

Myriangiales, genera incertae sedis

Phaeosclera Sigler, Tsuneda \& J.W. Carmich. 1981 (H)

Sarcinomyces Lindner 1898 (H)

Zopfiaceae G. Arnaud ex D. Hawksw. 1992

Cladosporium-like, anamorphic Zopfia Rabenh. $1874(\mathbf{H})$

Subclass Pleosporomycetidae C.L. Schoch, Spatafora, Crous \& Shoemaker

Pleosporales Luttrell ex M.E. Barr

Amniculicolaceae Yin. Zhang, C.L. Schoch, J.

Fourn., Crous \& K.D. Hyde

Anguillospora Ingold 1942 (H)

Spirosphaera Beverw. 1953 (H)

\section{Cucurbitariaceae G. Winter}

Camarosporium Schulzer 1870, anamorphic Cucurbitaria Gray 1821 (C)

Coniothyrium-like, anamorphic Curreya Sacc. 1883 (C)

Diplodia-like, anamorphic Cucurbitaria Gray 1821 (C)

?Megaloseptoria Naumov 1925, anamorphic Cucurbitaria Gray 1821 (C)

?Phaeoseptoria Speg. 1908, anamorphic Rhytidiella Zalasky 1968 (C)

Phialophorophoma Linder 1944 (C)

Phoma Sacc. 1880 (C)

Pleurophoma Höhn. 1914 (C)

Pleurostromella Petr. 1922, anamorphic Cucurbitaria Gray 1821 and Gibberidea Fuckel 1870 (C)

Pyrenochaeta De Not. 1849, anamorphic Cucurbitaria Gray 1821 (C)

Pyrenochaetopsis Gruyter, Aveskamp, \& Verkley, 2010 (C)

Syntholus A.W. Ramaley \& M.E. Barr 1997, anamorphic Syncarpella Theiss. \& Syd. 1915 (C)

Diademaceae Shoemaker \& C.E. Babc. Alternaria-like, anamorphic Comoclathris
Clem. 1909 (H)*

Fusicladiella Höhn. 1919, anamorphic Didymosphaeria Fuckel 1870 (H)

Didymellaceae Gruyter, Aveskamp \& Verkley

Ascochyta Lib. 1830, anamorphic Didymella Sacc. ex D. Sacc. 1880 (C)

Ampelomyces Ces. ex Schltdl. 1852 (C)

Boeremia Aveskamp, Gruyter \& Verkley 2010 (C)

Chaetasbolisia Speg. 1918 (C)

Dactuliochaeta G.L. Hartm. \& J.B. Sinclair 1988, anamorphic Didymella Sacc. $1880(\mathbf{C})$

Microsphaeropsis Höhn. 1917 (C)

Peyronellaea Gold. ex Togliani 1952 (C)

Phoma Sacc. 1880, anamorphic Didymella Sacc. ex D. Sacc. 1880 (C)

Piggotia Berk. \& Broome 1851, possibly anamorphic Platychora Petr. 1925 (C)

Pithomyces Berk. \& Broome 1875, anamorphic Leptosphaerulina McAlpine 1902 (H)

Stagonosporopsis Died. 1912, anamorphic Didymella Sacc. ex D. Sacc. 1880 (C) (=Ascochyta Lib. 1830)

Didymosphaeriaceae Munk

Cytoplea Bizz. \& Sacc. 1885, anamorphic Roussoëlla Sacc. 1888 (C)

Dothidotthiaceae Crous \& A.J.L. Phillips

?Dothiorella Sacc. 1880, anamorphic Dothidotthia Höhn. 1918 (C)

Thyrostroma Höhn. 1911, anamorphic Dothidotthia Höhn. 1918 (H)

Hypsostromataceae Huhndorf

Pleurophomopsis-like, anamorphic Hypsostroma Huhndorf (C)

Lentitheciaceae Yin. Zhang, C.L. Schoch, J. Fourn., Crous \& K.D. Hyde

Stagonospora (Sacc.) Sacc. 1884 (C)

Leptosphaeriaceae M.E. Barr

Camposporium Harkn. 1884, anamorphic Leptosphaeria Ces. \& De Not. 1863 (H)

Coniothyrium Corda 1840, anamorphic Leptosphaeria Ces. \& De Not. 1863 (C) Coniothyrium-like, anamorphic Neophaeo- 
sphaeria M.P.S. Câmara, M.E. Palm \& A.W. Ramaley 2003 (C)

Phoma Sacc. 1880, anamorphic Leptosphaeria Ces. \& De Not. 1863 (C)

Plenodomus Preuss 1851 (C)

Pyrenochaeta De Not. 1849, possibly anamorphic Leptosphaeria Ces. \& De Not. 1863 (C)

Lindgomycetaceae K. Hiray., Kaz. Tanaka \& Shearer

Taeniolella S. Hughes 1958 (H)

Lolia Abdel-Aziz \& Abdel-Whab 2010 (C)*

Lophiostomataceae Sacc.

Anguillospora Ingold 1942, anamorphic Massarina Sacc. 1883 (H)

Ascochyta Lib. 1830, anamorphic Trichometasphaeria Munk 1953 (C)

Periconia Tode 1791 anamorphic Lophiostoma Ces. \& De Not. 1863 (H)

Pleuorphomopsis-like, anamorphic Lophiostoma Ces. \& De Not. 1863 (C)

Pyricularia Sacc. 1880, anamorphic Massarina Sacc. $1883(\mathbf{H})$

Tumularia Descals \& Marvanová 1987(H)

Massariaceae Nitschke

?Aplosporella-like, anamorphic Dubitatio Speg. 1882 (C)

Myxocyclus Riess 1852, anamorphic Massaria De Not. 1844 (C)

Torula Pers. 1794 (H)

Massarinaceae Munk

Aquaticheirospora Kodsueb \& W.H. Ho 2007 (H)

Ceratophoma Höhn. 1917, anamorphic Massarina Sacc. 1883 (C)

Cheirosporium L. Cai \& K.D. Hyde 2008 (H)

Clavariopsis De Wild. 1895 (H)

Helminthosporium Link 1809, anamorphic Splanchnonema Corda 1829 (H)

Neottiosporina Subram. 1961 (C)

Pseudodictyosporium Matsush. 1971 (H)

Melanommataceae G. Winter

Aposphaeria Sacc. 1880 (C)

Beverwykella Tubaki 1975 (H)

Exosporiella P. Karst. 1892, anamorphic
Anomalemma Sivan. 1983 (H)

Nigrolentilocus R.F. Castañeda \& Heredia 2001, anamorphic Melanomma Nitschke ex Fuckel 1870 (H)

Monodictys S. Hughes 1958, anamorphic Ohleria Fuckel 1868 (H)

Monotosporella S. Hughes 1958 (H)

Pyrenochaeta De Not. 1849, anamorphic Byssosphaeria Cooke 1879 (C)

Sporidesmiella P.M. Kirk 1982 (H)

Montagnulaceae M.E. Barr

Aschersonia Mont. 1848, anamorphic Montagnula Berl. 1896 (C)

Microdiplodia Allesch. 1901, possibly anamorphic Karstenula Speg. 1879 (C)

Microsphaeropsis Höhn. 1917, anamorphic Paraphaeosphaeria O.E. Erikss. 1967 (C)

Paraconiothyrium Verkley 2004, possibly anamorphic Paraphaeosphaeria O.E. Erikss. 1967 (C)

Phaeosphaeriaceae M.E. Barr

Amarenographium O.E. Erikss. 1982, anamorphic Amarenomyces O.E. Erikss. 1981 (recently synonymised with Phaeosphaeria I. Miyake 1909) (C)

Ampelomyces Ces. ex Schltdl. 1852 (C)

Chaetosphaeronema Moesz 1915 (C)

Coniothyrium Corda 1840, anamorphic Ophiobolus Riess 1854 (C)

Harpophora W. Gams 2000, anamorphic anamorphic Ophiobolus Riess 1854 (H)

Hendersonia-like, anamorphic Austropleospora R.G. Shivas \& L. Morin 2010 and Phaeosphaeria I. Miyake 1909 (C)

Mauginiella Cavara 1925 (H)

Neosetophoma Gruyter, Aveskamp \& Verkley 2010 (C)

?Parahendersonia A.W. Ramaley 1995, anamorphic Chaetoplea (Sacc.) Clem. 1931 (C)

Paraphoma Morgan-Jones \& J.F. White 1983 (C)

Phaeoseptoria Speg. 1908, anamorphic Phaeosphaeria I. Miyake 1909 (C)

Phaeostagonospora A.W. Ramaley 1997, anamorphic Phaeosphaeriopsis M.P.S. Câmara, M.E. Palm \& A.W. Ramaley 2003 (C)

Phoma Sacc. 1880, anamorphic Ophio- 
bolus Riess 1854 (C)

Rhabdospora (Durieu \& Mont. ex Sacc.) Sacc. 1884, anamorphic Ophiobolus Riess 1854 (C)

Scolecosporiella Petr. 1921, anamorphic Ophiosphaerella Speg. and Phaeosphaeria I. Miyake 1909 (C)

Setophoma Gruyter, Aveskamp \& Verkley 2010 (C)

?Sphaerellopsis Cooke 1883, anamorphic Eudarluca Speg. 1908 (C)

Stagonospora (Sacc.) Sacc. 1884, anamorphic Phaeosphaeria I. Miyake 1909 (C)

Tiarospora Sacc. \& Marchal 1885 (C)

Pleomassariaceae M.E. Barr

Ceuthodiplospora Died. 1912, anamorphic Splanchnonema Corda (C)

Corynespora Güssow 1906, anamorphic Pleomassaria Speg. 1880 (H)

Myxocyclus Riess 1852, anamorphic Splanchnonema Corda 1829 (C)

?Prosthemium Kunze 1817, anamorphic Pleomassaria Speg. 1880 (C)

Scolicosporium Lib. ex Roum. 1880, anamorphic Asteromassaria Höhn. 1917 (C)

Shearia Petr. 1924, anamorphic Pleomassaria Speg. 1880 (C)

Stegonsporium Corda 1827, anamorphic Splanchnonema Corda 1829 (C)

Pleosporaceae Nitschke

Alternaria Nees 1816, anamorphic Lewia M.E. Barr \& E.G. Simmons 1986 (H)*

Alternaria-like anamorphic Clathrospora Rabenh. 1857 (H)

Alternariaster E.G. Simmons 2007 (H)

Brachycladium Corda 1838, anamorphic Crivellia Shoemaker \& Inderbitzin 2006 (H)

Bipolaris Shoemaker 1959, anamorphic Cochliobolus Drechsler 193 (H)

Chalastospora E.G. Simmons 2007 (H)

Corynespora Güssow 1906, anamorphic Pyrenophora Fr. 1849 (H)

Curvularia Boedijn 1933, anamorphic Cochliobolus Drechsler 1934 (H)

Dactuliophora C.L. Leakey 1964 (H)

Dendryphiella Bubák \& Ranoj. 1914, anamorphic Pleospora Rabenh. ex Ces. \& De Not. 1863 (H)
Dendryphion Wallr. 1833, anamorphic Pleospora Rabenh. ex Ces. \& De Not. 1863 (H)

Dictyosporium Corda 1836 (H)

Digitodesmium P.M. Kirk 1981 (H)

Drechslera S. Ito 1930, anamorphic Pyrenophora Fr. 1849 (H)

Edenia M.C. González, Anaya, Glenn, Saucedo \& Hanlin 2007 (H)

Embellisia E.G. Simmons 1971, anamorphic Lewia M.E. Barr \& E.G. Simmons (H)

Exserohilum K.J. Leonard \& Suggs 1974, anamorphic Setosphaeria K.J. Leonard \& Suggs 1974 (H)

Gibbago E.G. Simmons 1986 (H)

Lemonniera De Wild. 1894 (H)

Marielliottia Shoemaker 1999, anamorphic Pyrenophora Fr. 1849 (H)

Nimbya E.G. Simmons 1989, anamorphic Macrospora Fuckel 1870 (H)

Prathoda Subram. 1956 (H)

Sinomyces Yong Wang bis \& X.G. Zhang 2011 (H)

Stemphylium Wallr. 1833, anamorphic Pleospora Rabenh. ex Ces. \& De Not. 1863 (H)

Teretispora E.G. Simmons 2007 (H)

Ulocladium Preuss 1851, anamorphic Lasiobotrys Kunze 1823 (H)

Undifilum Pryor, Creamer, Shoemaker, McLain-Romero \& Hambl. 2009 (H)

Sporormiaceae Munk

?Amorosia Mantle \& D. Hawksw. 2006 (H)

Phoma Sacc. 1880 (C)

Phoma-like, anamorphic Westerdykella Stolk 1955 (C)

Teichosporaceae M.E. Barr

Coniothyrium-like, anamorphic Immothia M.E. Barr (C)

Tetraplosphaeriaceae Kaz. Tanaka \& K. Hiray Pseudotetraploa Kaz. Tanaka \& K. Hirayama 2009 (H)

Quadricrura Kaz. Tanaka, K. Hirayama \& Sat. Hatak. 2009 (H)

Tetraploa Berk. \& Broome 1850, anamorphic Tetraplosphaeria Kaz. Tanaka \& K. Hirayama 2009 (H) 
Tetraploa-like, anamorphic Triplosphaeria Kaz. Tanaka \& K. Hirayama 2009 and Polyplosphaeria Kaz. Tanaka \& K. Hirayama 2009 (H)

Trematosphaeriaceae K.D. Hyde, Yin. Zhang, Suetrong \& E.B.G. Jones

Hyphopodia-like, anamorphic Trematosphaeria Fuckel, Jb. nassau. Ver. Naturk (C)*

Pleosporales, genera incertae sedis

Berkleasmium Zobel 1854 (H)

Briansuttonia R.F. Castañeda, Minter \& Saikawa 2004 (H)

Cerebella Ces. 1851 (H)

Cheiromoniliophora Tzean \& J.L. Chen 1990 (H)

Diplococcium Grove 1885 (H)

Ellisembia Subram. 1992 (H)

Epicoccum Link 1815 (H)

Fusculina Crous \& Summerell 2006 (C)

Mycocentrospora Deighton 1972 (H)

Mycopappus Redhead \& G.P. White 1985 anamorphic Mycodidymella C.Z. Wei, Y. Harada \& Katum. 1998 (H)

Noosia Crous, R.G. Shivas \& McTaggart 2011 (H)

Ochrocladosporium Crous \& U. Braun 2007 (H)

Phoma Sacc. 1880 (C)

Periconia Tode 1791(H)

Pleurophomopsis Petr. 1924, anamorphic Astrosphaeriella Syd. \& P. Syd. 1913 (C)

Polyschema H.P. Upadhyay 1966 (H)

Pseudochaetosphaeronema Punith. 1979 (C)

Pseudopassalora Crous 2011 (H)

Repetophragma Subram. 1992 (H)*

?Scleroramularia Batzer \& Crous 2011 (H)

Sclerostagonospora Höhn. 1917 (C)

Sirodesmium De Not. 1849 (C)

Spiroplana Voglmayr, M.J. Park \& H.D. Shin 2011 (H)

Sporidesmium Link 1809 (C)

Versicolorisporium Sat. Hatak., Kaz.

Tanaka \& Y. Harada 2008 (C)

Ybotromyces Rulamort 1986 (H)

Dothideales genera incertae sedis
Mycosphere Doi 10.5943/mycosphere/3/2/5

Atramixtia Tsuneda, M.L. Davey \& Currah 2011 (H)

Celosporium Tsuneda \& Davey 2010 (H)

Diplococcium Grove 1885, anamorphic Otthia Nitschke ex Fuckel 1870 (H)

Pleosporomycetidae, genera incertae sedis

Acrogenospora M.B. Ellis 1971, anamorphic Farlowiella Sacc. 1891(H)

Dothideomycetes, orders incertae sedis

Acrospermales Minter, Peredo \& A.T. Watson

Acrospermaceae Fuckel

Dactylaria Sacc. 1880, anamorphic Acrospermum Tode 1783 (H)

Gonatophragmium Deighton 1969, anamorphic Acrospermum Tode 1790 (H)

Botryosphaeriales C.L. Schoch, Crous \& Shoemaker

Botryosphaeriaceae Theiss. \& H. Syd.

Alysidiella Crous 2006 (H)

Aplosporella Speg. 1880 (C)

Arthrinium Kunze 1817, anamorphic Leptoguignardia E. Müll. (H)

Bahusutrabeeja Subram. \& Bhat 1977 (H)

Barriopsis A.J.L. Phillips, A. Alves \& Crous 2008 (C)

Dichomera Cooke 1878 (C)

Diplodia Fr. 1834 (C)

Diplodia-like, anamorphic Neodeightonia C. Booth 1970 and Saccharata Denman \& Crous 2004 (C)

Dothichiza-like, anamorphic Leptoguignardia E. Müll. 1955 (C)

Dothiorella Sacc. 1880, anamorphic Spencermartinsia A.J.L. Phillips, A. Alves $\&$ Crous 2008 (C)

Fusicoccum Corda 1829, anamorphic Botryosphaeria Ces. \& De Not. 1863 (C)

Fusicoccum-like, anamorphic Melanops Nitschke ex Fuckel 1870 and Saccharata Denman \& Crous 2004 (C)

Hormonema Lagerb. \& Melin 1927, anamorphic Guignardia Viala \& Ravaz $1892(\mathbf{H})$

Lasiodiplodia Ellis \& Everh. 1896 (C)

Leptodothiorella Höhn. 1918, anamorphic Guignardia Viala \& Ravaz 1892 (C)

Macrophomina Petr. 1923 (C)

Microdiplodia Allesch. 1901 (C) 
Neofusicoccum Crous, Slippers \& A.J.L. Phillips 2006, with Botryosphaeria-like teleomorphs (C)

Neoscytalidium Crous \& Slippers 2006 (H)

Phyllosticta Pers. 1818, anamorphic Guignardia Viala \& Ravaz 1892 (C)

Pseudofusicoccum Mohali, Slippers \& M.J. Wingf. 2006 (C)

Sphaeropsis Sacc. 1880 (C)

Thyrostroma Höhn. 1911, anamorphic Dothidotthia Höhn. 1918 (H)

Botryosphaeriales, genera incertae sedis

Camarosporium Schulzer 1870 (C)

Dichomera Cooke 1878 (C)

Hendersonula Speg. 1880 (C)

Hysteriales Lindau

Hysteriaceae Chevall.

Aposphaeria-like, anamorphic Gloniopsis De Not. 1847 and Hysterobrevium E. Boehm \& C.L. Schoch 2010 (C)

Coniosporium Link 1809, anamorphic Hysterium Pers. 1797 (H)

Plenodomus Preuss 1851, anamorphic Psiloglonium Höhn. 1918 (C)

Septonema Corda 1837, anamorphic Oedohysterium E. Boehm \& C.L. Schoch 2009 (H)

Sporidesmium Link 1809, anamorphic Psiloglonium Höhn. 1918 (H)

Jahnulales Pang, Abdel-Wahab, ElSharouney, E.B.G. Jones \& Sivichai

Aliquandostipitaceae Inderbitzin

Brachiosphaera Nawawi 1976 (H)

Mytilinidiales Boehm, C.L. Schoch \& Spatafora

Gloniaceae (Corda) Boehm, C.L. Schoch \& Spatafora

Cenococcum Moug. \& Fr. 1829 (C)

Cleistonium Speer 1986, anamorphic Glonium Mühl. 1813 (C)

Mytilinidiaceae Kirschst.

Camaroglobulus Speer 1986, anamorphic Mytilinidion Duby 1862 (C)

Chalara (Corda) Rabenh. 1844, anamorphic Quasiconcha M.E. Barr \& M. Blackw. 1981 (H)*
Mycosphere Doi 10.5943/mycosphere/3/2/5

?Monotosporella S. Hughes 1958, anamorphic Actidium Fr. 1815 (H)

Papulospora Preuss 1851, anamorphic Lophium Fr. 1818 (H)

Septonema Corda 1837, anamorphic Mytilinidion Duby 1862 (H)

Taeniolella S. Hughes 1958, anamorphic Mytilinidion Duby 1861 (H)

Jahnulales genera incertae sedis

Xylomyces Goos, R.D. Brooks \& Lamore 1977 (H)

Patellariales D. Hawksw. \& O.E. Erikss.

Patellariaceae Corda

Aposphaeria-like, anamorphic Rhytidhysteron Speg. 1881 (C)

Diplodia-like, anamorphic Rhytidhysteron Speg. 1881 (C)

Venturiales Yin. Zhang, C.L. Schoch \& K.D. Hyde

Venturiaceae E. Müll. \& Arx ex M.E. Barr Acroconidiellina (Berk. \& Broome) M.B. Ellis 1971, anamorphic Acantharia Theiss. \& Syd. (H)*

Bactrodesmium Cooke 1883, anamorphic Phragmogibbera Samuels \& Rogerson 1990 (H)

Dictyodochium Sivan. 1984, anamorphic Gibbera Fr. 1825 (H)

?Didymochora Hohn. 1918, anamorphic Atopospora Petr. 1925 (C)

Cladosporium-like, anamorphic Apiosporina Höhn. 1910 (H)

Fusicladium Bonord. 1851, anamorphic Apiosporina Höhn. 1910, Caproventuria Braun 1998 and Venturia Sacc. $1882(\mathbf{H})$

Fusicladium-like, anamorphic Acantharia Theiss. \& Syd 1918 and Protoventuria Berl. \& Sacc. 1887 (H)

Fusicladosporium Partr. \& Morgan-Jones 2003, anamorphic Venturia Sacc. $1882(\mathbf{H})$

Helicodendron Peyronel 1918 anamorphic Tyrannosorus Unter. \& Malloch 1995 (H)

Pithosira Petr. 1949 (H)

Spilocaea Fr. 1819, anamorphic Venturia Sacc. 1882 (C)

Spilodochium Syd. 1927 anamorphic Pseu- 
doparodiella F. Stevens 1927 (H)

Stigmina-like, Sacc. 1980 anamorphic Aca-ntharia Theiss. \& Syd 1918 and Gib-bera Fr. 1825 (H)

Sympoventuriaceae Yin. Zhang, C.L. Schoch $\&$ K.D. Hyde

Fusicladium-like (H)

Sympodiella-like, anamorphic Sympoventuria Crous \& Seifert $(\mathbf{H}) *$

Veronaeopsis Arzanlou \& Crous 2007 (H)*

Dothideomycetes, families incertae sedis Ascoporiaceae Kutorga \& D. Hawksw.

Plectophomella-like, anamorphic Pseudosolidum Lloyd 1923 (H)

\section{Asterinaceae Hansf.}

?Acarella Syd. 1927, anamorphic Morenoina Theiss. 1913 (C)

Asterostomella Speg. 1886, anamorphic Asterina Lév. 1845 (C)

Asterostromina Bat. \& A.F. Vital 1957, anamorphic Asterodothis Theiss. (C)

Asterostomula Theiss. 1916, anamorphic Echidnodes Theiss. \& Syd. 1918 (C)

Bahusakala Subram. 1958 (H)

?Clasterosporium Schwein. 1832, anamorphic Eupelte Syd. 1924 (H)

Clasterosporium Schwein. 1832, anamorphic Asterina Lév. 1845, Asterodothis Theiss.1912, Yamamotoa Bat. 1960 and Lembosia Lév. 1845 (H)

?Elachopeltis Syd. 1927, anamorphic Aphanopeltis Syd. 1927 (C)

Eriothyrium Speg. 1888, anamorphic Aster-inema Bat. \& Gayão 1953 (C)*

Leprieurina G. Arnaud 1918, anamorphic Prillieuxina G. Arnaud 1918 (C)

Mahanteshamomyces Hosag. \& C.K. Biju 2004 (C)

?Peltasterella Bat. \& H. Maia 1959, anamorphic Yamamotoa Bat. 1960 (C)

Pirozynskia Subram. 1972, anamorphic Eupelte Syd. 1924 (H)

Septoidium G. Arnaud 1921, anamorphic Eupelte Syd. 1924 (H)

Septothyrella Höhn. 1911, anamorphic Uleothyrium Petr. 1929 (C)

Shivamyces Hosag. 2004 (H)
Sirothyriella Höhn. 1910, anamorphic Morenoina Thesis. 1913 (C)

?Thyrinula Petr. \& Syd. 1924, anamorphic Aulographina Arx \& E. Müll. 1960 (C)

Triposporium Corda 1837, anamorphic Batistinula Arx 1960 (H)

Corynesporascaceae Sivan.

Corynespora Güssow 1906, anamorphic Corynesporasca Sivan. (H)

Dacampiaceae Körb.

Cyclothyrium Petr. 1923, anamorphic Polycoccum Saut. ex Körb. 1865 (C)

Lecythophora-like, anamorphic Munkovalsaria Aptroot 1995 (H)

Englerulaceae Henn.

Capnodiastrum Speg. 1886, anamorphic Englerula Henn. 1904 and Rhytidenglerula Höhn. 1918 (C)

Digitosarcinella S. Hughes 1984, anamorphic Schiffnerula Höhn. 1909 (H)

Mitteriella Syd. 1933, anamorphic Schiffnerula Höhn. 1909 (H)

Questieriella G. Arnaud ex S. Hughes 1983, anamorphic Schiffnerula Höhn. 1909 (H)

Sarcinella Sacc. 1880, anamorphic Schiffnerula Höhn. 1909 (H)*

Eremomycetaceae Malloch \& Cain

Arthrographis G. Cochet ex Sigler \& J.W. Carmich. 1976, anamorphic Eremomyces Malloch \& Cain 1971and Pithoascina Valmaseda, A.T. Martínez \& Barrasa 1987 (H)

Trichosporiella Kamyschko 1960, anamorphic Rhexothecium Samson \& Mouch. 1975 and Eremomyces Malloch \& Cain 1971 (H)

Euantennariaceae Hughes \& Corlett

Antennatula Fr. ex F. Strauss 1850, anamorphic Euantennaria Speg. and Strigopodia Bat. 1957 (H)

Capnokyma S. Hughes 1975 (H)

Capnophialophora S. Hughes 1966, anamorphic Strigopodia Bat. 1957 (H)

Hormisciomyces Bat. \& Nascim. 1957, anamorphic Euantennaria Speg. (H) 
Hyphosoma Syd. 1924, anamorphic Strigopodia Bat. 1957 (H)

Plokamidomyces Bat., C.A.A. Costa \& Cif. 1957, anamorphic Trichopeltheca Bat., C.A.A. Costa \& Cif. 1958 (H)

Racodium Fr. 1829, anamorphic Strigopodia Bat. 1957 (H)

Trichothallus F. Stevens 1925, anamorphic Trichopeltheca Bat., C.A.A. Costa \& Cif. 1958 (H)

Fenestellaceae M.E. Barr

Pleurostromella Petr. 1922, anamorphic Fenestella Tul. \& C. Tul. 1863 (C)

Leptopeltidaceae Höhn. ex Trotter

?Idriella P.E. Nelson \& S. Wilh. 1956, anamorphic Dothiopeltis E. Mull. 1957 (H)

Leptothyrium-like, anamorphic Leptopeltis Höhn. 1917 (C)

Meliolinaceae S. Hughes

Briania D.R. Reynolds 1989, anamorphic Meliolina Syd. \& P. Syd. 1914 (H)

Micropeltidaceae Clem. \& Shear

Cyclopeltella Petr. 1953, anamorphic Cyclopeltis Petr. 1953 (C)

Heliocephala V.G. Rao, K.A. Reddy \& de Hoog 1984 (H)*

Holubovaniella R.F. Castañeda 1985 (H)*

Sirothyriella Höhn. 1910, anamorphic Stomiopeltis Theiss. 1914 (C)

Sporidesmium-like, anamorphic Stomiopeltis Theiss. 1914 (as Akaropeltella M.L. Farr, 1972) (H)

Pycnothyriaceous taxa associated with species of Stomiopeltis anamorphic Micropeltis Mont.

\section{Microthyriaceae Sacc.}

Asterostomula Theiss. 1916, anamorphic Asterinella Theiss. 1912 (C)

Asteromella-like, anamorphic Asterinella Theiss. 1912 (C)

Hansfordiella S. Hughes 1951, anamorphic Trichothyrium Speg. 1889 (H)

Isthmospora F. Stevens 1918, anamorphic Trichothyrium Speg. 1889 (H)

Leptothyrium Kunze 1823, anamorphic
Mycosphere Doi 10.5943/mycosphere/3/2/5

Microthyrium Desm. 1841 (C)

Xenogliocladiopsis Crous \& W.B. Kendr. 1994, anamorphic Arnaudiella Petr. 1927 (H)

Zalerion R.T. Moore \& Meyers 196, anamorphic Microthyrium Desm. 1841 (H)

Parmulariaceae E. Müll. \& Arx ex M.E. Barr

Excipulariopsis P.M. Kirk \& Spooner 1982, anamorphic Kentingia Sivan. \& W.H. Hsieh 1989 (H)

?Melanoplaca Syd. \& P. Syd. 1917, anamorphic Dothidasteroma Höhn. 1909 (C)

Placomelan Cif. 1962, anamorphic Dothidasteroma Höhn. 1909 (C)

Parodiellaceae Theiss. \& H. Syd. ex M.E. Barr ?Ascochytopsis Henn. 1905, anamorphic Parodiella Speg. 1880 (C)

Parodiopsidaceae Toro

?Chuppia Deighton 1965, anamorphic Neoparodia Petr. \& Cif. 1932 (H)

Cicinnobella Henn. 1904, anamorphic Perisporiopsis Henn. 1904 (as Phaeodimeriella Speg. 1908) and Dimerium (Sacc. \& P. Syd.) Sacc. \& D. Sacc. 1905 (C)

Clasterosporium Schwein. 1832, anamorphic Balladyna Racib. 1900 (H)

Heterosporiopsis Petr. 1950, anamorphic 'Dimerium' (Sacc. \& P. Syd.) Sacc. \& D. Sacc. 1905, Exosporinella Bender 1932, possibly anamorphic Parodiellina Henn. ex G. Arnaud 1918 (H)

?Ophiotrichum Kunze 1849 anamorphic Leptomeliola Höhn. 1919 (H)

Sarcinella-like, anamorphic Neoparodia Petr. \& Cif. 1932 (H)

Septoidium G. Arnaud 1921, anamorphic Alina Racib. 1909, Perisporiopsis Henn. 1904, Parodiellina Henn. ex G. Arnaud 1918, Chevalieropsis G. Arnaud 1923, Ophioparodia Petr. \& Cif. 1932 and Pilgeriella Henn. 1900 (H)

Shivamyces Hosag. 2004, anamorphic Alina Racib. 1909 (H)

Tretospora M.B. Ellis 1976, anamorphic Balladyna Racib. and Balladynopsis 
Theiss. \& Syd. (H)

Planistromellaceae M.E. Barr

Aposphaeria-like, anamorphic Microcyclus Sacc., Syd. \& P. Syd. 1904 (C)

Fusicladium Bonord. 1851, anamorphic Microcyclus Sacc., Syd. \& P. Syd. 1904 (H)

Hyphospora A.W. Ramaley 1996, anamorphic Comminutispora A.W. Ramaley 1996 (H)

Kellermania Ellis \& Everh. 1885, anamorphic Planistroma A.W. Ramaley 1991 (C)

Lecanosticta Syd. 1922, anamorphic Eruptio M.E. Barr 1996 (C)

Pazschkeella Syd. \& P. Syd. 1901, anamorphic Microcyclus Sacc., Syd. \& P. Syd. 1904 (C)

Piptarthron Mont. ex Höhn. 1918, anamorphic Planistroma A.W. Ramaley 1991 (C)

Polystomellaceae Theiss. \& H. Syd.

Asteromella Pass. \& Thüm. 1880 (as Stictochorella Höhn. 1917), anamorphic Dothidella Speg. 1880 (C)

Lasmenia Speg. 1886, anamorphic Munkiella Speg. 1885 (C)

\section{Pseudoperisporiaceae Toro}

Chaetosticta Petr. \& Syd. 1925, anamorphic Lasiostemma Theiss., Syd. \& P. Syd. 1917 and ?Nematostoma Syd. \& P. Syd. 1914 (C)

Schizothyriaceae Höhn. ex Trotter, Sacc., D. Sacc. \& Traverso

Plenotrichaius Bat. \& Valle 1961, anamorphic Mycerema Bat., J.L. Bezerra \& Cavalc 1963 (C)

Zygophiala E.W. Mason 1945, anamorphic Schizothyrium Desm. 1849 (H)

\section{Tubeufiaceae M.E. Barr}

?Annellospermosporella P.R. Johnst. 1999, anamorphic Malacaria Syd. 1930 (H)

Aquaphila Goh, K.D. Hyde \& W.H. Ho 1998, anamorphic Tubeufia Penz. \& Sacc. $1898(\mathbf{H})^{*}$
Araneomyces Höhn. 1909, anamorphic Paranectriella (P. Henn. \& Sacc.) Höhn. (H)

Chionomyces Deighton \& Piroz. 1972, anamorphic Melioliphila Speg. 1924 (H)

Corynespora Güssow 1906, anamorphic Chaetosphaerulina I. Hino 1938 (H)

Eriomycopsis Speg. 1910, anamorphic Melioliphila Speg. 1924 and Byssocallis Syd. 1927 (H)

Guelichia Speg. 1886, anamorphic Puttemansia Henn. 1902 (H)

Helicoma Corda 1837, anamorphic Tubeufia Penz. \& Sacc. 1898, Thaxteriella Petr. 1924 and Chlamydotubeufia Boonmee \& K.D. Hyde 2011 (H)*

Helicoön Morgan $1892(\mathbf{H}) *$

Helicomyces Link 1809, anamorphic Acanthostigma De Not. (1863) (H)*

Helicosporium Nees 1816, anamorphic Acanthostigma De Not. (1863) and Tubeufia Penz. \& Sacc. 1898 (H)

Kamalomyces R.K. Verma, N. Sharma \& Soni 2008 (C)

Mirandina G. Arnaud ex Matsush. 1975, anamorphic Taphrophila Scheuer 1988 (H)*

Monodictys S. Hughes 1958, anamorphic Tubeufia Penz. \& Sacc. 1898 (H)

Monodictys-like, anamorphic Tubeufia Penz. \& Sacc. 1898 (H)

Moorella P. Rag. Rao \& D. Rao 1964, anamorphic Chaetosphaerulina I. Hino 1938 (H)

Pendulispora M.B. Ellis 1961, anamorphic Tubeufia Penz. \& Sacc. 1898 (H)

Peziotrichum (Sacc.) Lindau 1900, anamorphic Podonectria Petch 1921 (H)

Spermatoloncha Speg. 1908 (H)

Tetracrium Henn. 1902, anamorphic Puttemansia Henn. 1902 and Podonectria Petch 1921 (H)

Titaea Sacc. 1876, anamorphic Paranectriella (Henn. ex Sacc. \& D. Sacc.) Höhn. 1910 and Puttemansia Henn. 1902 (H)

Xenosporium Penz. \& Sacc. 1901, anamorphic Acanthostigmella Höhn. 1905 and Chaetosphaerulina I. Hino 1938 (as 
Thaxteriellopsis Sivan., Panwar \& S.J. Kaur 1977) (H)

Vizellaceae H.J. Swart

Chrysogloeum Petr. 1959, anamorphic Blasdalea Sacc. \& P. Syd. 1902 (C)

Manginula G. Arnaud 1918, anamorphic Vizella Sacc. 1883 (C)

Dothideomycetes, genera incertae sedis

Acrocalymma Alcorn \& J.A.G. Irwin (1987), anamorphic Massarina, however Massarina has been shown to be polyphyletic and its placement is therefore presently unclear $(\mathbf{C})$

Ampullifera Deighton 1960, anamorphic Teratoschaeta Bat. \& O.M. Fonseca (H)

Ascochyta-like, anamorphic Gilletiella Sacc. \& P. Syd. 1899 (C)

Asteromella Pass. \& Thüm. 1880 (C)

Bactrodesmium Cooke 1883, anamorphic Stuartella Fabre 1879 (H)

Brachyconidiella R.F. Castañeda \& W.B. Kendr. 1990 (H)

Camarosporula Petr. 1954, anamorphic Anthracostroma Petr. 1954 (C)

Chaetasbolisia Speg. 1918, anamorphic Pseudomorfea Punith. 1981 (C)

Cladoriella Crous 2006 (H)

Coniosporium Link 1809 (H)

Coronospora M.B. Ellis 1971, anamorphic Ascocoronospora Matsush. 2003 (H)

Cryomyces Selbmann, de Hoog, Mazzaglia, Friedmann \& Onofri 2005 (H)

?Cyclothyrium Petr. 1923, anamorphic Thyridaria Sacc. 1875 (C)

Dendryphiopsis S. Hughes 1953, anamorphic Kirschsteiniothelia D. Hawksw. 1985 (H)

Diederichia D. Hawksw. 2003 (C)

Dilophospora Desm. 1840, anamorphic Lidophia J. Walker \& B. Sutton 1974 (C)

Diplodia-like, anamorphic Otthia Nitschke ex Fuckel 1870 (C)

Disculina Höhn. 1916 (C)

Funbolia Crous \& Seifert 2011 (H)

Hansfordiellopsis Deighton 1960, anamorphic Koordersiella Höhn. 1909 (H)
Helicodendron Peyronel 1918, anamorphic Tyrannosorus Unter. \& Malloch 1995 (H)

Hiospira R.T. Moore 1962, anamorphic Brooksia Hansf. 1956 (H)

Iledon Samuels \& J.D. Rogers 1986, anamorphic Botryohypoxylon Samuels \& J.D. Rogers 1986 (C)

Megaloseptoria Naumov 1925 (C)

Mirandina G. Arnaud ex Matsush. 1975, anamorphic Chaetonectrioides Matsush. 1996 (H)

Monodictys S. Hughes 1958 (H)

Peltaster Syd. \& P. Syd. 1917 (C)

Phaeothecoidiella Batzer \& Crous 2010 (H)

Phanerococculus Cif. 1954, anamorphic Koordersiella Höhn. 1909 (C)

Phialophora-like, anamorphic Tirisporella E.B.G. Jones, K.D. Hyde \& Alias 1996 (H)

Phoma-like, anamorphic Tremateia Kohlm., Volkm.-Kohlm. \& O.E. Erikss. 1995 (C)

Placosphaeria (De Not.) Sacc. 1880 (C)

Plectopycnis Bat. \& A.F. Vital 1959 (C)

Pleurostromella Petr. 1922, anamorphic Gibberidea Fuckel 1870 (C)

Pseudorobillarda M. Morelet 1968 (C)

Pycnopleiospora C.Z. Wei, Y. Harada \& Katum. 1997, anamorphic Pseudodidymella C.Z. Wei, Y. Harada \& Katum. 1997 (H)

Rhizopycnis D.F. Farr 1998 (C)

Scleroconidioma Tsuneda, Currah \& Thormann 2000 (H)

Septoriella Oudem. 1889 (C)

Troposporella P. Karst. 1892 (H)

Class Eurotiomycetes Tehler ex O.E. Eriksson \& K. Winka

Subclass Chaetothyriomycetidae Doweld

Chaetothyriales M.E. Barr

Chaetothyriaceae Hansf. ex M.E. Barr

?Ciferrioxyphium Bat. \& H. Maia 1963, anamorphic Aithaloderma P. Syd. (H)*

Cyphellophora G.A. de Vries 1962 (H)

Leptoxyphium Speg. 1918, anamorphic Aithaloderma P. Syd. 1913 (H)*

Merismella Syd. 1927, anamorphic Chaet- 
Mycosphere Doi 10.5943/mycosphere/3/2/5

othyrium Speg. 1888 (C)

Stanhughesia Constant. 1989, anamorphic Ceramothyrium Bat. \& H. Maia 1956 (H)

Tripospermum Speg. 1918, anamorphic Phragmocapnias Theiss. \& Syd. 1918, Trichomerium Speg. 1918 and Triposporiopsis W. Yamam. 1955 (H)*

Vonarxia Bat. 1960 (H)

Herpotrichiellaceae Munk

Brycekendrickomyces Crous \& M.J. Wingf. 2009 (H)

Cladophialophora Borelli 1980, anamorphic Capronia Sacc. 1883 (H)

Exophiala J.W. Carmich. 1966, anamorphic Capronia Sacc. 1883 (H)

Fonsecaea Negroni 1936, anamorphic Capronia Sacc. 1883 (H)

Metulocladosporiella Crous, Schroers, J.Z. Groenew., U. Braun \& K. Schub. 2006 (H)

Nadsoniella Issatsch. 1914 (H)

Phaeococcomyces de Hoog 1979 (H)

Phaeoramularia Munt.-Cvetk. 1960, anamorphic Capronia Sacc.1883 (H)

Phialophora Medlar 1915 (H)

Racodium Pers. 1794, anamorphic Capronia Sacc. 1883 (H)

Rhinocladiella Nannf. 1934, anamorphic Capronia Sacc. 1883 (H)

Sorocybe Fr. 1849 (H)

Thysanorea Arzanlou, W. Gams \& Crous 2007 (H)

Veronaea Cif. \& Montemart. 1957 (H)

Chaetothyriales M.E. Barr, genera incertae sedis

Coniosporium Link 1809, the type species is considered as Sirodesmium De Not. 1849 (H)

Phaeoannellomyces McGinnis \& Schell $1985(\mathbf{H})(=$ Hortaea $)$

Staninwardia B. Sutton 1971 (C)

Strelitziana Arzanlou \& Crous 2006 (H)

Pyrenulales, genera incertae sedis

Lyromma Bat. \& H. Maia 1965 (C)

Chaetothyriomycetidae, families incertae sedis Rhynchostomataceae Winka \& O. E. Erikss.
Arthropycnis Constant. 1992, anamorphic Rhynchostoma P. Karst. (C)

Strigulaceae Zahlbr.

Discosiella Syd. \& P. Syd. 1912, anamorphic Strigula Fr. 1823 (C)

Chaetothyriomycetidae, genera incertae sedis Peyronelia Cif. \& Gonz. Frag. 1927, anamorphic Glyphium Nitschke ex F. Lehm. 1886 (H)

Phaeomoniella Crous \& W. Gams 2000 (H)

Rhabdospora-like, anamorphic Dolabra C. Booth \& W.P. Ting 1964 (C)

Taeniolella S. Hughes 1958, anamorphic Glyphium Nitschke ex F. Lehm. 1886 (H)

Subclass Eurotiomycetidae Geiser \& Lutzoni

Coryneliales Seaver \& Chardon

Coryneliaceae Sacc. ex Berl. \& Voglino

Exophiala J.W. Carmich. 1966, anamorphic Bicornispora Checa, Barrasa, M.N. Blanco \& A.T. Martínez 1996 (H)

Eurotiales G.W. Martin ex Benny \& Kimbr.

Monascaceae J. Schröt.

Basipetospora G.T. Cole \& W.B. Kendr. 1968, anamorphic Monascus Tiegh. 1884 (H)

Fraseriella Cif. \& A.M. Corte 1957, anamorphic Xeromyces Fraser $(\mathbf{H})$

Trichocomaceae E. Fisch.

Aspergillus P. Micheli ex Link 1809, anamorphic Dichlaena Durieu \& Mont. 1849, Emericella Berk. \& Broome 1857, Eurotium Link 1809, Fennellia B.J. Wiley \& E.G. Simmons 1973, Hemicarpenteles A.K. Sarbhoy \& Elphick 1968, Neopetromyces Frisvad \& Samson 2000, Neosartorya Malloch \& Cain 1973, Petromyces Malloch \& Cain 1973 and Warcupiella Subram. $1972(\mathbf{H})^{*}$

Geosmithia J.Pitt 1979, anamorphic, Chromocleista Yaguchi \& Udagawa 1993 and Talaromyces C.R. Benj. 1955 (H)

Isaria Pers. 1794, anamorphic Byssochlamys Westling (H) 
Merimbla Pitt 1979, anamorphic Talaromyces C.R. Benj. 1955 (H)

Paecilomyces Bainier 1907, anamorphic Byssochlamys Westling 1909 and Talaromyces C.R. Benj. 1955 (H)

Paecilomyces-like, anamorphic Coonemeria Mouch. 1997 (H)

Penicillium Link 1809, anamorphic Eupenicillium F. Ludw. 1892, Talaromyces C.R. Benj. 1955, Dichlaena Durieu \& Mont. 1849 and Trichocoma Jungh. 1838 (H)

Phialosimplex Sigler, Deanna A. Sutton, Gibas, Summerb. \& Iwen 2010 (H)

Polypaecilum G. Sm. 1961, anamorphic Dactylomyces Sopp 1912, Dichotomomyces Saito ex D.B.Scott 1970 and Thermoascus Miehe 1907 (H)

Pseudocordyceps Hauman 1936, anamorphic Penicilliopsis Solms 1887 (H)

Sagenomella W. Gams 1978, anamorphic Sagenoma Stolk \& G.F. Orr 1974 and Talaromyces C.R. Benj. 1955 (H)

Sarophorum Syd. \& P. Syd. 1916, anamorphic Penicilliopsis Solms 1887 (H)

Stilbodendron Syd. \& P. Syd. 1916, anamorphic Penicilliopsis Solms 1887 (H)

Stilbothamnium Henn. 1896 (H)

Thermomyces Tsikl. 1899 (H)

Thysanophora W.B. Kendr. 1961 (H)

Torulomyces Delitsch 1943, anamorphic Eupenicillium F. Ludw. 1892 (H)

Eurotiomycetidae genera incertae sedis

Penicillium Link 1809, anamorphic Paratalaromyces Matsush. 2003 (H)

Onygenales Cif. ex Benny \& Kimbr.

Ajellomycetaceae Untereiner, J.A. Scott \& Sigler

Blastomyces Gilchrist \& W.R. Stokes 1898, anamorphic Ajellomyces McDonough \& A.L. Lewis 1968 (H)

Chrysosporium Corda 1833, anamorphic Ajellomyces McDonough \& A.L. Lewis 1968 (H)

Emmonsia Cif. \& Montemart. 1959, anamorphic Ajellomyces McDonough \& A.L. Lewis 1968 (H)

Histoplasma Darling 1906, anamorphic Ajellomyces McDonough \& A.L. Lewis 1968 (H)
Mycosphere Doi 10.5943/mycosphere/3/2/5

Lacazia Taborda, V.A. Taborda \& McGinnis 1999 (H)

Paracoccidioides F.P. Almeida 1930 (H)

Arachnomycetaceae Gibas, Sigler \& Currah

Onychocola Sigler 1990, anamorphic Arachnomyces Massee \& E.S. Salmon 1902 (H)

\section{Gymnoascaceae Baran.}

Chrysosporium-like, anamorphic Orromyces B. Sur \& G.R. Ghosh 1987 (H)

Malbranchea Sacc. 1882, anamorphic Byssoonygena Guarro, Punsola \& Cano 1987 and Gymnoascus Baran. 1872 (H)

Oncocladium Wallr. 1833, anamorphic Gymnoascus Baran. 1872 (H)

\section{Onygenaceae Berk.}

?Arthrographis G. Cochet ex Sigler \& J.W. Carmich. 1976, anamorphic Arachnotheca Arx 1971 (H)

Chrysosporium Corda 1833 anamorphic Amauroascus J. Schröt., Aphanoascus Zukal 1890, Apinisia La Touche 1968, Arachnotheca Arx 1971, Nannizziopsis Currah 1985, ?Polytolypa J.A. Scott \& Malloch 1993, Pectinotrichum Varsavsky \& G.F. Orr 1971, ?Renispora Sigler \& J.W. Carmich. 1979 (H)

Coccidioides G.W. Stiles 1896 (H)

Malbranchea Sacc. 1882, anamorphic Auxarthron G.F. Orr \& Kuehn 1963, Byssoonygena Guarro, Punsola \& Cano 1987 and Uncinocarpus Sigler \& G.F. Orr 1976 (H)

Myriodontium Samson \& Polon. 1978, anamorphic Neoarachnotheca Ulfig, Cano \& Guarro 1997 (H)

Sporendonema Desm. 1827, anamorphic Onygena Pers. 1800 (H)

Arthrodermataceae Currah

Chrysosporium Corda 1833, anamorphic Shanorella R.K. Benj. 1956, Arthroderma Curr. 1860 and Ctenomyces Eidam 1880 (H)

Chrysosporium Corda 1833, anamorphic Epidermophyton Sabour. 1907 (H)

Microsporum Gruby 1843, anamorphic Nannizzia Stockdale 1961 and Arthroderma Curr. 1860 (H) 
Myceliophthora Costantin 1892, anamorphic Arthroderma Curr. 1860

Trichophyton Malmsten 1848, anamorphic Arthroderma Curr. 1860 (H)

Ascosphaeraceae L.S. Olive \& Spiltoir

Chrysosporium-like, anamorphic Ascosphaera L.S. Olive \& Spiltoir 1955 and Bettsia Skou 1972 (H)

Onygenales, genera incertae sedis

Arthropsis Sigler, M.T. Dunn \& J.W. Carmich. 1982 (H)

Lacazia Taborda, V.A. Taborda \& McGinnis 1999 (H)

Subclass Mycocaliciomycetidae Tibell

Mycocaliciales Tibell \& Wedin

Mycocaliciaceae A.F.W. Schmid

Asterophoma D. Hawksw. 1981, anamorphic Chaenothecopsis Vain. 1927 (C)

Catenomycopsis Tibell \& Constant. 1991, anamorphic Chaenothecopsis Vain. 1927 (H)

Phialophora-like, anamorphic Chaenothecopsis Vain. 1927 and Mycocalicium Vain. 1890 (H)

\section{Class Laboulbeniomycetes Engler}

Laboulbeniales Engler

Coreomycetopsis Thaxt. 1920 (H)

Muiaria Thaxt. 1914 (H)

Tetrameronycha Speg. ex W. Rossi \& M. Blackw. 1990 (H)

Pyxidiophorales P.F. Cannon

Pyxidiophoraceae Arnold

Chalara (Corda) Rabenh. 1844 anamorphic Pyxidiophora Bref. \& Tavel $1891(\mathbf{H})^{*}$

Gliocephalis Matr. 1899 (H)

Pleurocatena G. Arnaud ex Aramb., Gamundí, W. Gams \& G.R.W. Arnold 2007, anamorphic Pyxidiophora Bref. \& Tavel 1891 (H)

Thaxteriola Speg. 1918, anamorphic Pyxidiophora Bref. \& Tavel 1891 (H)

Class Lecanoromycetes O.E. Erikss. \& Winka

Subclass Ostropomycetidae Reeb, Lutzoni \& Cl. Roux
Agyriales Clem. \& Shear

Trapeliaceae M. Choisy ex Hertel

Epithyrium (Sacc.) Trotter 1931, anamorphic Sarea Fr. 1825 (C)

Pycnidiella Höhn. 1915, anamorphic Sarea Fr. 1825 (C)

Ostropales Nannf.

Odontotremataceae D. Hawksw. \& Sherwood Pycnidial anamorph associated with Claviradulomyces P.R. Johnst., D.C. Park, H.C. Evans, R.W. Barreto \& D.J. Soares 2010

Stictidaceae Fr.

?Coleophoma-like, anamorphic Stictophacidium Rehm 1888 (C)

?Ebollia Minter \& Caine 1980, anamorphic Stictophacidium Rehm 1888 (C)

Phacidiella-like, anamorphic Acarosporina Sherwood 1977 (C)

Stictospora Cif. 1957, anamorphic Stictis Pers. 1800 (C)

Ostropales, genera incertae sedis

Glomerobolus Kohlm. \& Volkm.-Kohlm. 1996 (H)

Subclass Lecanoromycetidae P.M. Kirk, P.F. Cannon, J.C. David \& Stalpers ex Miadl., Lutzoni \& Lumbsch

Lecanorales Nannf.

Pilocarpaceae Zahlbr.

Pyriomyces Bat. \& H. Maia 1965, anamorphic Byssoloma Trevis. 1853 (C)

Szczawinskia A. Funk 1984 (C)

Stereocaulaceae Chevall.

Lepraria Ach. 1803 (C)

Lecanorales, genera incertae sedis

Leprocaulon Nyl. 1878 (C)

Vouauxiomyces Dyko \& D. Hawks. 1979, anamorphic Abrothallus De Not. 1845

(C)

Class Leotiomycetes O.E. Erikss. \& Winka

Cyttariales Luttr. ex Gamund1'

Cyttariaceae Speg.

Cytariella Palm 1932, anamorphic Cytta- 
ria Berk. 1842 (C)

Erysiphales Gwynne-Vaughan

Erysiphaceae Tul. \& C. Tul.

Oidiopsis Scalia 1902, anamorphic Leveillula G. Arnaud 1921 (H)

Oidium Link 1824, anamorphic Arthrocladiella Vassilkov 1960, Blumeria Golovin ex Speer 1975, Brasiliomyces Viégas 1944, Cystotheca Berk. \& M.A. Curtis 1860, Erysiphe R. Hedw. ex DC. 1805, Golovinomyces (U. Braun) V.P. Gelyuta 1988, Medusosphaera Golovin \& Gamalizk. 1962, Microsphaera Lév. 1851, U. Braun 1999, Podosphaera Kunze 1823, Sawadaea Miyabe 1914, Sphaerotheca Lév. 1851, Uncinula Lév. 1851 and Uncinuliella R.Y. Zheng \& G.Q. Chen 1979 (H)

Ovulariopsis Pat. \& Har. 1900, anamorphic Phyllactinia Lév. 1851 (H)

Streptopodium R.Y. Zheng \& G.Q. Chen 1978, anamorphic Pleochaeta Sacc. \& Speg. 1881 and Phyllactinia Lév. 1851 (H)

\section{Helotiales Nannf. \\ Bulgariaceae Fr.}

Crinula Fr. 1821, anamorphic Holwaya Sacc. 1889 (H)

Endomelaconium Petr. 1940, anamorphic Bulgaria Fr. 1822 (C)

\section{Dermateaceae Fr.}

Anguillospora Ingold 1942, anamorphic Mollisia (Fr.) P. Karst. 1871 (H)

Cadophora Lagerb. \& Melin 1927, anamorphic Mollisia (Fr.) P. Karst. 1871, Pyrenopeziza Fuckel 1870 and Pseudopezicula Korf 1975 (H)

Cadophora-like, anamorphic Phaeomollisia T.N. Sieber \& Grünig 2009 (H)

Casaresia Gonz. Frag. 1920, anamorphic Mollisia (Fr.) P. Karst. 1871 (H)

Chondropodium-like, anamorphic Waltonia Saho 1970 (C)

Corniculariella P. Karst. 1884, anamorphic Dermea Fr. 1825 and Durandiella Seaver 1932 (C)

Cryptocline Petr. 1924, anamorphic Trochila Fr. 1849 (C)

Cryptosporiopsis Bubák \& Kabát 1912, anamorphic Neofabraea H.S. Jacks.
1913, Pezicula Tul. \& C. Tul. 1865 and ?Ocellaria (Tul. \& C. Tul.) P. Karst. 1871 (C)

Cryptosympodula Verkley 1999, anamorphic Scleropezicula Verkley 1999 (C)

Cylindrocolla Bonord. 1851, anamorphic Calloria Fr. 1836 (H)

Cylindrosporium Grev. 1822, anamorphic Pyrenopeziza Fuckel 1870 (C)

Cystodendron Bubák 1914, anamorphic Mollisia (Fr.) P. Karst. 1871 Belonopsis (Sacc.) Rehm 1891 and Niptera Fr. 1849 (H)

Diplosporonema Höhn. 1917, anamorphic Pyrenopeziza Fuckel 1917 (C)

Discogloeum Petr. 1923, anamorphic Spilopodia Boud. 1885 (C)

Entomosporium Lév. 1856, anamorphic Diplocarpon F.A. Wolf 1912 (C)

Eriosporella Höhn. 1916, possibly anamorphic Laetinaevia Nannf. 1932 (C)

Foveostroma DiCosmo 1978, anamorphic Dermea Fr. 1825 (C)

Gelatinosporium Peck 1873, anamorphic Dermea Fr. 1825 and Durandiella Seaver 1932 (C)

Glarea Bills \& Paláez 1999 (H)

Gloeosporidiella Petr. 1921, anamorphic Drepanopeziza (Kleb.) Höhn. 1917 and Pseudopeziza Fuckel 1870 (C)

Hainesia Ellis \& Sacc. 1884, anamorphic Discohainesia Nannf. 1932 (C)

Helicodendron Peyronel 1918, anamorphic Mollisia (Fr.) P. Karst. 1871 (H)

Laetinaevia Nannf. 1932, anamorphic Naeviopsis B. Hein 1976 (C)

Marssonina Magnus 1906, anamorphic Diplocarpon F.A. Wolf 1912 \& Drepanopeziza (Kleb.) Höhn. 1917 (C)

Melanodiscus Höhn. 1918, anamorphic Spilopodia Boud. 1885 (H)

Microgloeum Petr. 1922, anamorphic Blumeriella Arx 1961 (C)

Monostichella Höhn. 1916, anamorphic Drepanopeziza (Kleb.) Höhn. 1917 (C)

Myrioconium Syd. \& P. Syd. 1912, anamorphic Dibeloniella Nannf. 1932 (H)

Phacidiella P. Karst. 1884, anamorphic Pyrenopeziza Fuckel 1870 (C)

Phialocephala W.B. Kendr. 1961 anamorphic Phaeomollisia T.N. Sieber \& Grünig 2009 (H) 
Phialocephala-like, anamorphic Phaeomollisia T.N. Sieber \& Grünig 2009 (H)

Phialophora-like, anamorphic Dibeloniella Nannf. 1932 (H)

Phloeosporella Höhn. 1924, anamorphic Blumeriella Arx 1961 (C)

Phlyctema Desm. 1847, anamorphic Neofabraea H.S. Jacks. 1913 (C)

Pilidium Kunze 1823, anamorphic Discohainesia Nannf. 1932 (C)

Rhynchosporium Heinsen ex A.B. Frank $1897(\mathbf{H}) *$

?Sporonema Desm. 1847, anamorphic Leptotrochila P. Karst. 1871 (C)

Trichosporiella Kamyschko 1960, anamorphic Laetinaevia Nannf. 1932 (H)

Trimmatostroma Corda 1837 (H)

Xenochalara M.J. Wingf. \& Crous 2000 (H)

\section{Helotiaceae Rehm}

Alatospora Ingold 1942 (H)

Anguillospora Ingold 1942, anamorphic Hymenoscyphus Gray 1821 (H)

Articulospora Ingold 1942, anamorphic Hymenoscyphus Gray 1821(H)

Ascoconidium Seaver 1942, anamorphic Sageria A. Funk 1975 (H)

Aureohyphozyma Hosoya \& Y. Otani 1995 anamorph of Gelatinipulvinella Hosoya \& Y. Otani (Hosoay Y Otani 1995) (H)

Bloxamia Berk. \& Broome 1854, anamorphic Bisporella Sacc. 1884 (H)

Bothrodiscus Shear 1907, anamorphic Ascocalyx Naumov 1926 (C)

Brunchorstia Erikss. 1891, anamorphic Gremmeniella M. Morelet 1969 (C)

?Bryophytomyces Cif. 1953, anamorphic Hymenoscyphus Gray 1821 (H)

Cercosporella Sacc. 1880, anamorphic Heterosphaeria Grev. 1824 (H)

Coryne Nees 1816, anamorphic Ascocoryne J.W. Groves \& D.E. Wilson 1967 (H)

Cystodendron Bubák 1914, anamorphic Bisporella Sacc. 1884 (H)

?Cystotricha Berk. \& Broome 1850, anamorphic Xylogramma Wallr. 1833 (C)

Deltosperma W.Y. Zhuang 1988, anamorphic Unguiculariopsis Rehm 1909
(C)

Dendrostilbella Höhn. 1905, anamorphic Claussenomyces Kirschst. 1923 (H)

Dichaenopsella Petr. 1952, type is anamorphic Godroniopsis Diehl \& E.K. Cash 1929 (C)

Digitosporium Gremmen 1953, anamorphic Crumenulopsis J.W. Groves 1969 (C)

Dimorphospora Tubaki 1958, anamorphic Hymenoscyphus Gray 1821 (H)

Dothiorina Höhn. 1911, anamorphic Chlorociboria Seaver ex Ramamurthi, Korf \& L.R. Batra 1958 (C)

Endomelaconium Petr. 1940, anamorphic Austrocenangium Gamundí 1997 (C)

Eriomycopsis Speg. 1910, anamorphic Calloriopsis Syd. \& P. Syd. 1917 (H)

Eustilbum Rabenh. 1864, anamorphic Bisporella Sacc. $1884(\mathbf{H})$

Fuckelia Bonord. 1864, anamorphic Godronia Moug. \& Lév. 1846 (C)

Geniculospora Sv. Nilsson ex Marvanová \& Sv. Nilsson 1971, anamorphic Hymenoscyphus Gray 1821 (H)

Gliomastix Guég. 1905 anamorphic Ascoclavulina Y. Otani 1974 (H)*

Heteropatella Fuckel 1874, anamorphic Heterosphaeria Grev. 1824 (C)

Hormonema-like, anamorphic Pragmopora A. Massal 1855 (H)

Idriella P.E. Nelson \& S. Wilh. 1956, anamorphic Hymenoscyphus Gray 1821 (H)

Linodochium Höhn. 1909, anamorphic Pseudohelotium Fuckel 1870 (H)

Monodictys S. Hughes 1958 anamorphic Hymenoscyphus Gray 1821 (H)

Periperidium Darker 1963, anamorphic Micraspis Darker 1963 (C)

Phialophora-like, anamorphic Pseudopezicula Korf 1975 (H)

Pragmopycnis B. Sutton \& A. Funk 1975, anamorphic Pragmopora A. Massal 1855 (C)

?Pseudaegerita J.L. Crane \& Schokn. 1981, anamorphic Claussenomyces Kirschst. 1923 (H)

Pseudospiropes M.B. Ellis 1971, anamorphic Strossmayeria Schulzer 1881 (H) 
Rhinocladiella-like, anamorphic Bioscypha Syd. 1927 (H)

Rhizothyrium Naumov 1915, anamorphic Rhizocalyx Petr. 1928 (C)

Scytalidium Pesante 1957 anamorphic Hymenoscyphus Gray 1821 (H)

Sirodothis Clem. 1909, anamorphic Tympanis Tode 1790 (C)

Sporonema Desm. 1847, anamorphic Godronia Moug. \& Lév. 1846 (C)

Symphyosirella Seifert 2009, anamorphic Symphyosirinia E.A. Ellis 1956 (H)

Titaeospora Bubák 1916, anamorphic Stamnaria Fuckel 1870 (C)

Topospora Fr. 1836, anamorphic Godronia Moug. \& Lév. 1846 (C)

Tricladium Ingold 1942, anamorphic Cudoniella Sacc. 1889 and Hymenoscyphus Gray 1821 (H)

Varicosporium W. Kegel 1906, anamorphic Hymenoscyphus Gray 1821 (H)

\section{Hemiphacidiaceae Korf}

Meria Vuill. 1896, anamorphic Rhabdocline Syd. 1922 (H)

Rhabdogloeum Syd. 1922, anamorphic Rhabdocline Syd. 1922 (C)

Rhabdogloeopsis Petr. 1925, anamorphic Sarcotrochila Höhn. 1917 (C)

\section{Hyaloscyphaceae Nannf.}

Acleistia Bayl. Ell. (1917) anamorphic Calycina Nees ex Gray 1821 (C)

Brefeldochium Verkley 2005, anamorphic Polydesmia Boud. 1885 (H)

Chaetochalara B. Sutton \& Piroz. 1965 (H)

Chalara (Corda) Rabenh. 1844, anamorphic Calycellina Höhn., Phaeoscypha Spooner 1984 and Tapesina Lambotte $1887(\mathbf{H})^{*}$

Cheiromycella Höhn. 1910, anamorphic Hyaloscypha Boud. 1885 (H)

Clathrosphaerina Beverw. 1951, anamorphic Hyaloscypha Boud. 1885 (H)

Haplographium Berk. \& Broome 1859, anamorphic Dematioscypha Svrček 1977 (H)

Mycoarthris Marvanová \& P.J. Fisher 2002 (H)
Mycosphere Doi 10.5943/mycosphere/3/2/5

Naemospora Roth ex Kuntze 1898, possibly anamorphic Lachnellula P. Karst. 1884 (C)

Phialophora-like, anamorphic Cistella Quél. and Hyalopeziza Fuckel 1870 (H)

Pseudaegerita J.L. Crane \& Schokn. 1981, anamorphic Hyaloscypha Boud. 1885 (H)

Septonema-like, anamorphic Ciliolarina Svrcek 1977 (H)

Tricladium Ingold 1942, anamorphic Hydrocina Scheuer 1991 (H)

\section{Leotiaceae Corda}

Anguillospora Ingold 1942, anamorphic Pezoloma Clem. 1909 (H)

Aureohyphozyma Hosoya \& Y. Otani 1995, anamorphic Gelatinipulvinella Hosoya \& Y. Otani 1995 (H)

Halenospora E.B.G. Jones 2009 (H)

Myrioconium-like, anamorphic Neobulgaria Petr. 1921 (H)

Phialophora-like, anamorphic Neobulgaria Petr. 1921 (H)

Loramycetaceae Dennis ex Digby \& Goos

Anguillospora Ingold 1942, anamorphic Loramyces W. Weston 1929 (H)

Phacidiaceae Fr.

Allantophomopsis Petr. 1925, anamorphic Phacidium Fr. 1815 (C)

Apostrasseria Nag Raj 1983, anamorphic Lophophacidium Lagerb. 1949 and Phacidium Fr. 1815 (C)

Blennoria Moug. \& Fr. 1825, anamorphic Phacidium Fr. 1815 (C)

Ceuthospora Grev. 1826, anamorphic Phacidium Fr. 1815 (C)

?Coma Nag Raj \& W.B. Kendr. 1972, anamorphic Ascocoma H.J. Swart (C)

Rutstroemiaceae Holst-Jensen, L.M. Kohn \& T. Schumacher

Helicodendron Peyronel 1918, anamorphic Lambertella Höhn. 1918 (H)

Myrioconium Syd. \& P. Syd. 1912, ?anamorphic Lanzia Sacc. 1884, Rutstroemia P. Karst. 1871, Scleromitrula 
S.Imai 1941, Lambertella Höhn. 1918 and Poculum Velen. 1934 (H)

Phialophora-like, anamorphic Rutstroemia P. Karst. 1871 (H)

Sclerotiniaceae Whetzel ex Whetzel

Acarosporium Bubák \& Vleugel ex Bubák 1911, anamorphic Pycnopeziza W.L. White \& Whetzel 1938 (C)

Amerosporium Speg. 1882, anamorphic Zoellneria Velen. 1934 (C)

Amphobotrys Hennebert 1973, anamorphic Botryotinia Whetzel 1945 (H)

Botrytis P. Micheli ex Pers. 1794, anamorphic Botryotinia Whetzel 1945 (H)

Cristulariella Höhn. 1916, anamorphic Nervostroma Narumi \& Y. Harada 2006 (H)

Haradamyces Masuya, Kusunoki, Kosaka \& Aikawa 2009 (H)

Hinomyces Narumi \& Y. Harada 2006, anamorphic Grovesinia M.N. Cline, J.L. Crane \& S.D. Cline 1983 (H)

Monilia Bonord. 1851, anamorphic Monilinia Honey 1928 and Phaeosclerotinia Hori 1916 (H)

Mycopappus Redhead \& G.P. White 1985, anamorphic Redheadia Y. Suto \& Suyama 2005 (H)

Myrioconium Syd. \& P. Syd. 1912, anamorphic Ciboria Fuckel 1870, Ciborinia Whetzel 1945, (Fr.) P. Karst. 1871, Martininia Dumont \& Korf 1970, Myriosclerotinia N.F. Buchw. 1947, Sclerotinia Fuckel 1870 Stromatinia (Boud.) Boud. 1907 and Monilinia Honey 1928 (H)

Ovulitis N.F. Buchw. 1970, anamorphic Ovulinia F.A. Weiss 1940 (H)

Sclerotium Tode 1790, anamorphic Myriosclerotinia N.F. Buchw. 1947, Sclerotinia Fuckel 1870 and Stromatinia (Boud.) Boud. 1907 (H)

Septotis N.F. Buchw. ex Arx 1970, anamorphic Septotinia Whetzel ex J.W. Groves \& M.E. Elliott 1961 (H)

Streptobotrys Hennebert 1973, anamorphic Streptotinia Whetzel 1945 (H)

Valdensia Peyronel 1923, anamorphic Valdensinia Peyronel 1953 (H)
Verrucobotrys Hennebert 1973, anamorphic Seaverinia Whetzel 1945 (H)

\section{Vibrisseaceae Korf}

Acephala Grünig \& T.N. Sieber 2005 (H)

Anavirga B. Sutton 1975, anamorphic Vibrissea Fr. 1822 (H)

Phialocephala W.B. Kendr. 1961, some possibly anamorphic Vibrissea Fr. 1822 (H)

Phialophora-like, anamorphic Chlorovibrissea L.M. Kohn 1989 (H)

Helotiales, genera incertae sedis

Catenulifera Hosoya 2002, anamorphic Hyphodiscus Kirschst. 1906 (H)

Chalara (Corda) Rabenh. 1844 (H)*

Clathrosporium Nawawi \& Kuthub. 1987 (H)

Crucellisporium M.L. Farr 1968 (C)

Cystotricha Berk. \& Broome 1850 (C)

Dactylaria Sacc. 1880 (H)

Diplococcium Grove 1885 (H)

Endoconidium Prill. \& Delacr. 1891, anamorphic Gloeotinia M. Wilson, Noble \& E.G. Gray 1954 (H)

Fontanospora Dyko 1978 (H)

Fulvoflamma Crous 2006 (H)

Glarea Bills \& Paláez 1999 (H)

Helgardia Crous \& W. Gams 2003, anamorphic Oculimacula Crous \& W. Gams 2003 (H)

Humicolopsis Cabral \& S. Marchand 1976 (H)

Hyalodendriella Crous 2007 (H)

Hysteropezizella Höhn. 1917 (C)

Lemonniera De Wild. 1894 (H)

Leptodontidium de Hoog 1979 (H)

Libartania Nag Raj 1979 (C)

Margaritispora Ingold 1942 (H)

Meliniomyces Hambl. \& Sigler 2005 (H)

Mirandina G. Arnaud ex Matsush. 1975 (H)

Monochaetiellopsis B. Sutton \& DiCosmo 1977, anamorphic Hypnotheca Tommerup 1970 (C)

Mycochaetophora Hara \& Ogawa 1931 (H)

Neozythia Petr. 1958 (C)

Rhexocercosporidium U. Braun 1994 (H)

Rhizocladosporium Crous \& U. Braun 
2007 (H)

Rhodesia Grove 1937 (C)

Scytalidium Pesante 1957 (H)

Sphaerographium Sacc. 1884 (C)

Spirosphaera Beverw. 1953 (H)

Tetracladium De Wild. 1893 (H)

Thedgonia B. Sutton 1973 (H)

Trimmatostroma Corda 1837 (H)

Variocladium Descals \& Marvanová 1999

(H)

Xenopolyscytalum Crous 2011('2010’) (H)

Rhytismatales M.E. Barr ex Minter

Ascodichaenaceae D. Hawksw. \& Sherwood

Dichaenopsella Petr. 1952, anamorphic Ascodichaena Butin 1977 (C)

Macroallantina Speer 1987, anamorphic Delpinoina Kuntze, 1891 (C)

Polymorphum Chevall. 1822, anamorphic Ascodichaena Butin 1977 (C)

Cryptomycetaceae Höhn.

?Phacidiopycnis Potebnia 1912, anamorphic Potebniamyces Smerlis 1962 (C)

Rhytismataceae Chevall.

Conostroma Moesz 1921, anamorphic Colpoma Wallr. 1833 (C)

Crandallia Ellis \& Sacc. 1897, anamorphic Bifusella Höhn. 1917 and Duplicaria Fuckel 1870 (C)

Cryocaligula Minter 1986, anamorphic Ploioderma Darker. 1967 (C)

Hysterodiscula Petr. 1942, anamorphic Duplicaria Fuckel 1870 (C)

Leptostroma Fr. 1815, anamorphic Lophodermium Chevall. 1826 (C)

Melasmia Lév. 1846, anamorphic Rhytisma Fr. 1818 (C)

Tryblidiopycnis Höhn. 1918, anamorphic Tryblidiopsis P. Karst. 1871 (C)

Rhytismatales, genera incertae sedis

Fuligomyces Morgan-Jones \& Kamal 1984 (H)

Uyucamyces H.C. Evans \& Minter 1985, anamorphic Ocotomyces H.C. Evans \& Minter 1985 (C)

Leotiomycetes, families incertae sedis Myxotrichaceae Currah
Geomyces Traaen 1914, anamorphic Pseudogymnoascus Raillo 1929 (H)

?Gymnostellatospora Udagawa, Uchiy. \& Kamiya 1993, anamorphic Pseudogymnoascus Raillo 1929 (C)

?Malbranchea Sacc. 1882, anamorphic Myxotrichum Kunze 1823 (H)

Oidiodendron Robak 1932, anamorphic Byssoascus Arx 1971 and Myxotrichum Kunze 1823 (H)

Leotiomycetes, genera incertae sedis

Brachyalara Réblová \& W. Gams 2011 (H)

Chaetomella Fuckel 1870 (C)

Collophora Damm \& Crous 2010 (C)

Eleutheromyces Fuckel 1870 (C)

Geniculospora Sv. Nilsson ex Marvanová \& Sv. Nilsson 1971 (H)

Hyphozyma de Hoog \& M.T. Sm. 1981 (H)

Infundichalara Réblová \& W. Gams 2011 (H)

Leohumicola N.L. Nick. 2005 (H)

Meliniomyces Hambl. \& Sigler 2005 (H)

Scytalidium Pesante 1957, anamorphic Xylogone Arx \& T. Nilsson 1969 (H)

Tetrachaetum Ingold 1942 (H)

Tiarosporella Höhn. 1924, anamorphic Darkera H.S. Whitney, J. Reid \& Piroz. 1975 (C)

Class Orbiliomycetes O.E. Erikss. \& Baral

Orbiliales Baral, O.E. Erikss., G. Marson \& E. Weber

Orbiliaceae Nannf.

Anguillospora Ingold 1942, anamorphic anamorphic Orbilia Fr. 1836 (H)

Arthrobotrys Corda 1939, anamorphic Orbilia Fr. 1836 (H)

Brachyphoris Juan Chen, L.L. Xu, B. Liu \& Xing Z. Liu 2007, anamorphic Hyalorbilia Baral \& G. Marson 2000 (H)

Dactylaria Sacc. 1880, anamorphic Orbilia Fr. 1836 (H)

Dactylella Grove 1884, anamorphic Orbilia Fr. 1836 (H)

Dactylellina M. Morelet 1968 (H)

Dicranidion Harkn. 1885, anamorphic Orbilia Fr. 1836 (H) 
Drechslerella Subram. 1964, anamorphic Orbilia Fr. 1836 (H)

Dwayaangam Subram. 1978, anamorphic Orbilia Fr. 1836 (H)

Gamsylella M. Scholler, Hagedorn \& A. Rubner 1999, anamorphic Orbilia Fr. 1836 (H)

Helicoön Morgan 1892, anamorphic Orbilia Fr. 1836 (H)

Idriella P.E. Nelson \& S. Wilh. 1956, anamorphic Orbilia Fr. 1836 (H)

Monacrosporium Oudem. 1885, anamorphic Orbilia Fr. 1836 (H)

Pseudotripoconidium Z.F. Yu \& K.Q. Zhang 2011, anamorphic Orbilia Fr. $1836(\mathbf{H}) *$

Tricellula Beverw. 1954, anamorphic Orbilia Fr. 1836 (H)

Tridentaria Preuss 1852 (H)

Trinacrium Riess 1852, anamorphic Orbilia Fr. 1836 (H)

Class Pezizomycetes sensu O.E. Erikss. \& Winka

Pezizales J. Schröt.

Ascobolaceae Boud. ex Sacc.

Papulospora Preuss 1851, anamorphic Ascobolus Pers. 1796 (H)

Papulospora-like, anamorphic Cubonia Sacc. 1889 (H)

Rhizostilbella Wolk 1914, anamorphic Ascobolus Pers. 1792 (H)

\section{Caloscyphaceae Harmaja}

Geniculodendron G.A. Salt 1974, anamorphic Caloscypha Boud. 1885 (H)

\section{Chorioactidaceae Pfister}

Kumanasamuha P.Rag. Rao \& D. Rao 1964, anamorphic Chorioactis Kupfer ex Eckblad 1968 (H)

Verticicladium Preuss 1851, anamorphic Desmazierella Lib. 1829 (H)

Morchellaceae Reichenb.

Costantinella Matr. 1892, anamorphic Morchella Dill. ex Pers. 1794 (H)

\section{Pezizaceae Dumort.}

Chromelosporium Corda 1833, anamor-
Mycosphere Doi 10.5943/mycosphere/3/2/5

phic Muciturbo P.H.B. Talbot 1989, Peziza Dill. ex Fr. 1822 and Plicaria Fuckel 1870 (H)

Glischroderma Fuckel 1870 (C)

Oedocephalum Preuss 1851, anamorphic Iodophanus Korf 1967 and Peziza Dill. ex Fr. 1822 (H)

Ostracoderma Fr. 1825, anamorphic Peziza Dill. ex Fr. 1822 (C)

\section{Pyronemataceae Corda}

Actinosporella Descals, Marvanová \& J. Webster 1999, anamorphic Miladina Svrček 1972 (H)

Ascorhizoctonia Chin S. Yang \& Korf 1985, anamorphic Tricharina Eckblad 1968 (H)

Basifimbria Subram. \& Lodha 1968, anamorphic Geopyxis (Pers.) Sacc. (H)

Complexipes C. Walker 1979, anamorphic Tricharina Eckblad 1968 and Wilcoxina Chin S. Yang \& Korf 1985 (H)

?Dichobotrys Hennebert 1973, anamorphic Pyropyxis Egger 1984 and Trichophaea Boud. 1885 (H)

Micronematobotrys Xiang Sun \& L.D. Guo 2010 (H)

?Molliardiomyces Paden 1984, anamorphic Stephensia Tul. \& C. Tul. 1851 (H)

?Scytalidium Pesante 1957, anamorph of Anthracobia Boud. 1885 (H)

Rhizinaceae Bonord.

Phymatotrichopsis Hennebert 1973 (H)

Sarcoscyphaceae LeGal ex Eckblad

Molliardiomyces Paden 1984, anamorphic Nanoscypha Denison 1972, Phillipsia Berk. 1881, Pithya Fuckel 1870 and Sarcoscypha (Fr.) Boud. 1885 (H)

Verticicladium Preuss 1851, anamorphic Desmazierella Lib. 1829 (H)

\section{Sarcosomataceae Kobayasi}

Conoplea Pers. 1797, anamorphic Urnula Fr. 1849 and Plectania Fuckel 1870 (H) Strumella Fr. 1849, anamorphic Urnula Fr. 1849 (H)

Verticicladium Preuss 1851, anamorphic 
Mycosphere Doi 10.5943/mycosphere/3/2/5

Sarcosoma Casp. 1891 (H)

Pezizales, genera incertae sedis

Cephaliophora Thaxt. 1903 (H)

Class Sordariomycetes sensu O.E. Erikss. \& Winka

Subclass Hypocreomycetidae O.E. Erikss. \& Winka

Coronophorales Nannf.

Chaetosphaerellaceae Huhndorf, A.N. Mill. \& F.A. Fernández

Oedemium Link 1824, anamorphic Chaetosphaerella E. Müll. \& C. Booth 1972 (H)

Veramycina Subram. 1995, anamorphic Chaetosphaerella E. Müll. \& C. Booth 1972 (H)

Nitschkiaceae (Fitzp.) Nannf.

Acremonium-like, anamorphic Acanthonitschkea Speg. (H)

Glomerellales Chadef. ex Réblová, W. Gams \& Seifert

Australiascaceae Réblová \& W. Gams

Monilochaetes Halst. ex Harter 1916, anamorphic Australiasca Sivan. \& Alcorn 2002 (H)*

Glomerellaceae Locq. ex Seifert \& W. Gams

Colletotrichum Corda 1831, anamorphic Glomerella Spauld. \& Schrenk 1903 (C)

Reticulascaceae Réblová \& W. Gams

Cylindrotrichum Bonord.1851, anamorphic Reticulascus Réblová \& W. Gams $2011(\mathbf{H})^{*}$

\section{Hypocreales Lindau}

Bionectriaceae Samuels \& Rossman

Acremonium-like, anamorphic Dimerosporiella Speg. 1908, Ijuhya Starbäck 1899, Hydropisphaera Dumont 1822, Lasionectria (Sacc.) Cooke 1884, Mycoarachis Malloch \& Cain 1970, Mycocitrus Moller 1901, Nectriella Nitschke ex Fuckel 1870, Nectriopsis Maire 1911, Nigrosabulum Malloch \& Cain 1970, Protocreopsis Yoshim. Doi 1977, Selinia P. Karst. 1876, and Stilbocrea Pat. 1900 and Verrucostoma
Hirooka, Tak. Kobay. \& Chaverri 2010 (H)

Albosynnema E.F. Morris 1967 (H)

Clonostachys Corda 1839, anamorphic Bionectria Speg. 1919 (H)

Dendrodochium-like, anamorphic Nectriella Nitschke ex Fuckel 1870 (C)

Didymostilbe Henn. 1902, anamorphic Peethambara Subram. \& Bhat 1978 (H)

Geosmithia J.Pitt 1979 (H)*

Gliocladium-like, anamorphic Roumegueriella Speg. 1880 (H)

Gliomastix Guég. 1905 (H)*

Gracilistilbella Seifert 2000, anamorphic Stilbocrea Pat. 1900 (H)

Kutilakesa Subram. 1956, anamorphic Nectriella Nitschke ex Fuckel 1870 (H)

Rhopalocladium Schroers, Samuels \& W. Gams 1999, anamorphic Nectriopsis Maire 1911 (H)

Sarocladium W. Gams \& D. Hawksw. 1976 (H)

Spicellum Nicot \& Roquebert 1976 (H)

Stanjemonium W. Gams, O'Donnell, Schroers \& M. Chr. 1999 (H)

Stilbella Lindau 1900 anamorphic Stilbocrea Pat. 1900 (H)

Trichothecium-like, anamorphic Heleococcum C.A. Jørg. 1922 (H)

Tubercularia-like, anamorphic Selinia P. Karst. 1876 (H)

Verticillium-like, anamorphic Nectriopsis Maire 1911 (H)

Vesicladiella Crous \& M.J. Wingf. 1994 (H)

Virgatospora Finley 1967, anamorphic Peethambara Subram. \& Bhat 1978 (H)

Clavicipitaceae (Lindau) Earle ex Rogerson

Acremonium-like, anamorphic Neobarya Lowen 1986 (H)

Albomyces I. Miyake 1908, anamorphic Aciculosporium I. Miyake (C)

Amphichordia Fr., 1825 (H)

Aschersonia Mont. 1848, anamorphic Hypocrella Sacc. and Moelleriella Bres. 1897 (C)

Blistum B. Sutton 1973, anamorphic Berkelella (Sacc.) Sacc. 1891 (H)

Calcarisporium Preuss 1851, anamorphic Neobarya Lowen $\mathbf{( H )}$

Corallocytostroma Y.N. Yu \& Z.Y. Zhang 
$1980(\mathbf{C})$

Diploöspora Grove 1916, anamorphic Neobarya Lowen 1986 (H)

Drechmeria W. Gams \& H.-B. Jansson 1985 (H)

Ephelis Fr. 1849, anamorphic Atkinsonella Diehl, Balansia Speg., 1885, Epichlö̈ (Fr.) Tul. \& C. Tul., 1865, Dothichlö̈ G.F. Atk. 1894, Heteroepichlö̈ E. Tanaka, C. Tanaka, Gafur \& Tsuda 2002, Nigrocornus Ryley \& Langdon 2003 and Myriogenospora G.F. Atk. 1894 (H)

Harposporium Lohde 1874, anamorphic Podocrella Seaver 1928 (H)

Hirsutella-like, anamorphic Orbiocrella D. Johnson, G.H. Sung, Hywel-Jones \& Spatafora 2009 and Moelleriella Bres. 1897 (H)

Lecanicillium W. Gams \& Zare 2001, anamorphic Hypocrella Sacc. 1878and Neobarya Lowen 1986 (H)

Metarhizium Sorokīn 1879, anamorphic Metacordyceps G.H. Sung, J.M. Sung, Hywel-Jones \& Spatafora 2007 (H)

?Munkia Speg. 1886, anamorphic Mycomalus A. Møller 1901 (H)

Neomunkia Petr. 1947 (H)

Neotyphodium Glenn, C.W. Bacon \& Hanlin 1996, anamorphic Epichlö̈ (Fr.) Tul. \& C. Tul. 1865 (H)

Nomuraea Maubl. 1903 (H)

Pleurodesmospora Samson, W. Gams \& H.C. Evans 1979 (H)

Pochonia Bat. \& O.M. Fonseca 1965 (H)

Polycephalomyces Kobayasi 1941, anamorphic Berkelella (Sacc.) Sacc. 1891 (H)

Pseudogibellula Samson \& H.C. Evans 1973, anamorphic Torrubiella Boud. 1885 (H)

Pseudomeria G.L. Barron 1980 (H)

Rotiferophthora G.L. Barron 1991 (H)

Sphacelia Lév. 1827, anamorphic Atkinsonella Diehl 1950 and Claviceps Tul. 1853 (H)

Ustilaginoidea Bref. 1895, anamorphic Villosiclava E. Tanaka \& C. Tanaka 2009 (H)

Cordycipitaceae Kreisel ex G.M. Sung, J.M. Sung, Hywel-Jones \& Spatafora
Akanthomyces Lebert 1858, anamorphic Cordyceps Fr. 1824 and Torrubiella Boud. 1885 (H)

Beauveria Vuill. 1912, anamorphic Cordyceps Fr. 1824 (H)*

Engyodontium de Hoog 1978 (H)

Gibellula Cavara 1894, anamorphic Cordyceps Fr. 1824 and Torrubiella Boud. 1885 (H)

Granulomanus de Hoog \& Samson 1978, anamorphic Torrubiella Boud. 1885 (H)

Hirsutella Pat. 1892, anamorphic Cordyceps Fr. 1824 and Cordycepioideus Stifler 1941 (H)

Isaria Pers. 1794, anamorphic Cordyceps Fr. 1824 and Torrubiella Boud. 1885 (H)

Lecanicillium W. Gams \& Zare 2001, anamorphic Cordyceps Fr. 1824 and Torrubiella Boud. 1885 (H)

Microhilum H.Y. Yip \& A.C. Rath 1989 (H)

Pseudogibellula Samson \& H.C. Evans 1973 (H)

Rotiferophthora G.L. Barron 1991 (H)

Septofusidium W. Gams 1971, anamorphic Cordyceps Fr. 1824 (H)

Simplicillium W. Gams \& Zare 2001, possibly anamorphic Torrubiella Boud. 1885 (H)

Tolypocladium W. Gams 1971, anamorphic Cordyceps Fr. 1824 (H)

\section{Hypocreaceae De Not.}

Acremonium-like, anamorphic Protocrea Petch 1937 and Pseudohypocrea Yoshim. Doi 1972 (H)

Arachnocrea Z. Moravec 1956, anamorphic Protocrea Petch 1937 and Sarawakus Lloyd 1924 (C)

Cladobotryum Nees 1816, anamorphic Hypomyces (Fr.) Tul. \& C. Tul. 1860 (H)*

Gliocladium Corda 1840, anamorphic Hypocrea Fr. 1825 and Sphaerostilbella (Henn.) Sacc. \& D. Sacc. 1905 (H)

Gliocladium-like, anamorphic Sarawakus Lloyd 1924 (H)

Mycogone Link 1809, anamorphic Hypomyces (Fr.) Tul. \& C. Tul. 1860 (H) 
Papulaspora-like, anamorphic Hypomyces (Fr.) Tul. \& C. Tul. 1860 (H)

Sepedonium Link 1809, anamorphic Hypomyces (Fr.) Tul. \& C. Tul. 1860 (H)

Sibirina G.R.W. Arnold 1970, anamorphic Hypomyces (Fr.) Tul. \& C. Tul. 1860 (H)

Stephanoma Wallr. 1833, anamorphic Hypomyces (Fr.) Tul. \& C. Tul. 1860 (H)

Stilbella Lindau 1900, anamorphic Hypocrea Fr. 1825 (H)

Stromatocrea W.B. Cooke 1952, anamorphic Hypocreopsis P. Karst. 1873 (H)

Trichoderma Pers. 1794, anamorphic Hypocrea Fr. 1825 and Podostroma P. Karst. 1892 (H)*

Trichoderma-like, anamorphic Sarawakus Lloyd 1924 (H)

Trichothecium-like, anamorphic Hypomyces (Fr.) Tul. \& C. Tul. 1860 (H)

Verticillium-like, anamorphic Aphysiostroma Barrasa, A.T. Martínez \& G. Moreno 1986, Sphaerostilbella Henn. 1901 and Coniochaeta (Sacc.) Cooke 1887 (H)

Verticimonosporium Matsush. 1971 (H)

Nectriaceae Tul. \& C. Tul.

Acremonium-like, anamorphic Cosmospora Rabenh. 1862, Nectria (Fr.) Fr. 1849 and Neocosmospora E.F. Sm. 1899 (H)

Actinostilbe Petch 1925, anamorphic Lanatonectria Samuels \& Rossman 1999 (H)

Antipodium Piroz. 1974, anamorphic Ophionectria Sacc. 1878 (H)

Aphanocladium W. Gams 1971 (H)

Atractium Link 1809 (H)

Calostilbella Höhn. 1919, anamorphic Calostilbe Sacc. \& Syd. 1902 (H)

Campylocarpon Halleen, Schroers \& Crous 2005 (H)

Chaetodochium Höhn. 1932, anamorphic Pseudonectria Seaver 1909 (H)

Chaetopsina Rambelli 1956, anamorphic Chaetopsinectria J. Luo \& W.Y. Zhuang and Cosmospora Rabenh. 1862 (H)

Curvicladiella Decock \& Crous 2006 (H)

Curvicladium Decock \& Crous 1998 (H)
Cyanochyta Höhn. 1915, anamorphic Gibberella Sacc. 1877 (C)

Cyanophomella Höhn. 1918, anamorphic Gibberella Sacc. 1877 (C)

Cylindrocarpon Wollenw. 1913, anamorphic Neonectria Wollenw. 1917, Rugonectria P.Chaverri \& Samuels 2011, Thelonectria P. Chaverri \& C.G. Salgado 2011, Ilyonectria P.Chaverri \& Samuels 2011 (H)*

Cylindrocladiella Boesew. 1982, anamorphic Cosmospora Rabenh. 1862 and Nectricladiella Crous \& C.L. Schoch 2000 (H)

Cylindrocladium Morgan 1892, anamorphic Calonectria De Not. 1867 (H)

Dacryoma Samuels 1988, anamorphic Nectria (Fr.) Fr. 1849 (H)

Dematiocladium Allegr., Aramb., Cazau \& Crous 2005 (H)

Dichomera Cooke 1878, anamorphic Gibberella Sacc. 1877 (C)

Flagellospora Ingold 1942, anamorphic Nectria (Fr.) Fr. 1849 (H)

Fusarium Link 1809, anamorphic Albonectria Rossman \& Samuels 1999, Cyanonectria Samuels \& Chaverri 2009, Gibberella Sacc. 1877, Corallomycetella Henn. 1904 and Haematonectria Samuels \& Nirenberg 1999 and unknown teleomorphs $(\mathbf{H})$ *

Fusarium-like, anamorphic, Geejayessia Schroers, Gräfenhan \& Seifert 2011 and Cyanonectria Samuels \& Chaverri 2009 (H)*

Fusicolla Bonord. 1851 (H)

Gliocephalotrichum J.J. Ellis \& Hesselt. 1962, anamorphic Leuconectria Rossman, Samuels \& Lowen 1993 (H)

Gliocladiopsis S.B. Saksena 1954, anamorphic Glionectria Crous \& S.L. Schoch 2000 (H)

Gyrostroma Naumov 1914, anamorphic Nectria (Fr.) Fr. 1849 (C)

Heliscus Sacc. 1880, anamorphic Neonectria Wollenw. 1917 . 1849 (H)

Macroconia (Wollenw.) Gräfenhan, Seifert \& Schroers 2011 (H)

Mariannaea G. Arnaud ex Samson 1974 anamorphic 'Nectria' (Fr.) Fr. 1849 and Cordyceps Fr. 1833 (H)* 
Microcera Desm. 1848, anamorphic Gibberella Sacc. 1877 (H)*

Mirandina G. Arnaud ex Matsush. 1975, anamorphic Chaetonectrioides Matsush. (H)

Nalanthamala Subram. 1956, anamorphic Rubrinectria Rossman \& Samuels 1999 (H)

Penicillifer Emden 1968, anamorphic Viridispora Samuels \& Rossman 1999 (H)

Pleurocolla Petr. 1924 (H)

Rhizostilbela Wolk 1914, anamorphic Corallomycetella Henn. 1904 (H)

Septofusidium W. Gams 1971 (H)

Stachybotryna Tubaki \& T. Yokoy. 1971, anamorphic 'Nectria' (Fr.) Fr. 1849 (H)

Stilbella Lindau 1900, anamorphic Cosmospora Rabenh. 1862 and Nectria (Fr.) Fr. 1849 (H)*

Tilachlidium Preuss 1851, anamorphic 'Pseudonectria' Seaver 1909 (H)

Tubercularia Tode 1790, anamorphic Nectria (Fr.) Fr. 1849 (H)*

Verticillium-like, anamorphic Cosmospora Rabenh. 1862 (H)

Volutella Fr. 1832, anamorphic Cosmospora Rabenh. 1862 and Pseudonectria Seaver 1909 (H)

Xenocylindrocladium Decock, Hennebert \& Crous 1997, anamorphic Xenocalonectria Crous \& C.L. Schoch 2000 (H)

Zythiostroma Höhn. ex Falck 1923, anamorphic Nectria (Fr.) Fr. 1849 (C)

Niessliaceae Kirschst.

Acremonium-like, anamorphic Trichosphaerella E. Bommer, M. Rousseau \& Sacc. 1891 and Valetoniellopsis Samuels \& M.E. Barr 1998 (H)

Custingophora-like, anamorphic Melanopsamma Niessl 1876 (H)

Monocillium S.B. Saksena 1955, anamorphic Hyaloseta A.W. Ramaley 2001 and Niesslia Auersw. 1869 (H)

Ophiocordycipitaceae G.H. Sung, J.M. Sung,

Hywel-Jones \& Spatafora

Chaunopycnis W. Gams 1979 (H)

Haptocillium W. Gams \& Zare 2001 anamorphic Ophiocordyceps Petch $1931(\mathbf{H})$
Mycosphere Doi 10.5943/mycosphere/3/2/5

Hirsutella Pat. 1892, anamorphic Ophiocordyceps Petch 1931 (H)

Hymenostilbe Petch 1931, anamorphic Ophiocordyceps Petch 1931 (H)

Paraisaria Samson \& B.L. Brady 1983, anamorphic Ophiocordyceps Petch 1931 (H)

Purpureocillium Luangsa-ard, HywelJones, Houbraken \& Samson 2011 (H)

Syngliocladium Petch 1932, anamorphic Ophiocordyceps Petch 1931(H)

Tolypocladium W. Gams 1971, anamorphic Elaphocordyceps G.H. Sung \& Spatafora 2007 (H)

Verticillium-like, anamorphic Elaphocordyceps G.H. Sung \& Spatafora 2007 (H)

Hypocreales, genera incertae sedis

Acremonium Link 1809 (H)*

Acremonium-like, anamorphic Emericellopsis J.F.H. Beyma 1940 and Leucosphaerina Arx 1987 (H)

Ascochytopsis-like, anamorphic Eucasphaeria Crous 2007 (C)

Cancellidium Tubaki 1975 (H)

Didymostilbe Henn. 1902, anamorphic Peethambara Subram. \& Bhat 1978 (H)

Escovopsis J.J. Muchovej \& Della Lucia 1990 (H)

Hapsidospora Malloch \& Cain 1970 (C)

Haptospora G.L. Barron 1991 (H)

Illosporiopsis D. Hawksw. 2001 (H)

Illosporium Mart. 1817 (H)

Imicles Shoemaker \& Hambl. 2001 (H)

Memnoniella Höhn. 1923 (H)

Myrothecium Tode 1790 (H)

Parasarcopodium Melnik, S.J. Lee \& Crous 2004 (H)

Pseudomicrodochium B. Sutton 1975 (H)

Sarocladium W. Gams \& D. Hawksw. 1976 (H)

Septomyrothecium Matsush. 1971, anamorphic Peethambara Subram. \& Bhat 1978 (H)

Sorosporella Sorokīn 1888 (H)

Spicellum Nicot \& Roquebert 1976 (H)

Sporothrix Hektoen \& C.F. Perkins 1901, anamorphic Leucosphaerina Arx 1987 (H)

Stachybotrys Corda 1837 (H) 
Stilbella Lindau 1900, anamorphic Emericellopsis J.F.H. Beyma 1940 and Valsonectria Speg. 1881 (H)

Tilachlidium Preuss 1851 (H)

Trichothecium Link 1809 (H)*

Lulworthiales Kohlm., Spatafora \& Volkm.Kohlm. 2000

Lulworthiaceae Kohlm., Spatafora \& Volkm.-

Kohlm. 2000

Anguillospora Ingold 1942, anamorphic Lindra I.M. Wilson 1956 (H)

Melanosporales N. Zhang \& M. Blackw.

Ceratostomataceae G. Winter

Acrospeira Berk. \& Broome 1857 (H)

Gonatobotrys Corda 1839, anamorphic Melanospora Corda 1837 (H)

Harzia Costantin 1888, anamorphic Melanospora Corda 1837 (H)

Papulaspora Preuss 1851, anamorphic Melanospora Corda 1837 and Microthecium Corda 1842 (H)

Proteophiala Cif. 1958, anamorphic Melanospora Corda 1837, Persiciospora P.F. Cannon \& D. Hawksw. 1982, Sphaerodes Clem. 1909, Pteridiosperma J.C. Krug \& Jeng 1979 (H)

[Sphaerodes Clem. 1909 is a teleomorph which was reported to produce ampulliform phialides on irregularly branched conidiophores diectrly on ascomata or on surrounding hyphae (Vujanovic \& Goh, 2009)]

Microascales Luttr. ex Benny \& Kimbr.

Chadefaudiellaceae Faurel \& Schotter ex Benny \& Kimbr.

Arthrographis-like, anamorphic Faurelina Locq.-Lin. 1975 (H)

Gondwanamycetaceae Réblová, W. Gams \& Seifert

Custingophora Stolk, Hennebert \& Klopotek 1968, anamorphic Gondwanamyces G.J. Marais \& M.J. Wingf. 1998 (H)*

Halosphaeriaceae E. Müll. \& Arx ex Kohlm. Cirrenalia Meyers \& R.T. Moore 1960 (H)
Clavariopsis-like, anamorphic Corollospora Werderm. 1922 (H)

Clavatospora Sv. Nilsson ex Marvanová \& Sv. Nilsson 1971, anamorphic Corollospora Werderm. 1922 (H)

Culcitalna Meyers \& R.T. Moore 1960, anamorphic Halosarpheia Kohlm. \& E. Kohlm. 1977 (H)

Halosigmoidea Nakagiri, K.L. Pang \& E.B.G. Jones 2009, anamorphic Corollospora Werderm. 1922 (H)

Humicola Traaen 1914, anamorphic Pseudolignincola Chatmala \& E.B.G. Jones 2006 and Lignincola Höhnk 1955 (H)

Monodictys S. Hughes 1958 anamorphic Nereiospora E.B.G. Jones, R.G. Johnson \& S.T. Moss $1983(\mathbf{H})$

Periconia Tode 1791, anamorphic Halosphaeria Linder 1944 (H)

Sigmoidea J.L. Crane 1968, anamorphic Corollospora Werderm. $1922(\mathbf{H})$

Trichocladium Harz 1871, anamorphic Halosphaeria Linder 1944 (H)

Varicosporina Meyers \& Kohlm. 1965, anamorphic Corollospora Werderm. 1922 (H)

Microascaceae Luttr. ex Malloch

Atrhrographis G. Cochet ex Sigler \& J.W. Carmich. 1976, anamorphic Canariomyces Arx (H)

Brachyconidiellopsis Decock, R.F. Castañeda \& Adhikari 2004 (H)

Cephalotrichum Link 1809, anamorphic Microascus Zukal 1885 (H)

Echinobotryum Corda 1831 (H)

Graphium Corda 1837, anamorphic Microascus Zukal 1885, Petriella Curzi 1930 and Pseudallescheria Negr. \& I. Fisch. 1944 (H)

Humicola Traaen 1914, anamorphic Lophotrichus R.K. Benj. 1949 (H)

Parascedosporium Gilgado, Gené, Cano \& Guarro 2007 (H)

Scedosporium Sacc. ex Castell. \& Chalm. 1919, anamorphic Pseudallescheria Negr. \& I. Fisch. 1944 and possibly Petriella Curzi 1930 (H)

Scopulariopsis Bainier 1907, anamorphic Microascus Zukal 1885 and ?Petriella Curzi 1930 and Pithoascus Arx 1973 (H) 
Trichurus Clem. 1896 (H)

Wardomyces F.T. Brooks \& Hansf. 1923, anamorphic Microascus Zukal 188 (H)

Wardomycopsis Udagawa \& Furuya 1978, anamorphic Microascus Zukal 1885 (H)

Microascales, genera incertae sedis

Bisporostilbella Brandsb. \& E.F. Morris 1971 (H)

Chalara-like, anamorphic Ceratocystis Ellis \& Halst. 1890 and Cornuvesica C.D. Viljoen, M.J. Wingf. \& K. Jacobs 2000 (H)

Gabarnaudia Samson \& W. Gams 1974, anamorphic Sphaeronaemella P. Karst. 1884 (H)

Sporendocladia G. Arnaud ex Nag Raj \& W.B. Kendr. 1975 (H)

Thielaviopsis Went 1893, anamorphic Ceratocystis Ellis \& Halst. 1890 (H)

Hypocreomycetidae, families incertae sedis

Plectosphaerellaceae W. Gams, Summerbell \& Zare

Acremonium Link 1809 (H)*

Acrostalagmus Corda $1838(\mathbf{H}) *$

Gibellulopsis Bat. \& H. Maia 1959 (H)

Musicillium Zare \& W. Gams 2007 (H)

Plectosporium M.E. Palm, W. Gams \& Nirenberg 1995, anamorphic Plectosphaerella Kleb. 1929 (H)

Stachylidium Link 1809 (H)*

Verticillium Nees 1816 (H)

Hypocreomycetidae, genera incertae sedis

Canalisporium Nawawi \& Kuthub. 1989, anamorphic Ascothailandia Sriindrasutdhi, Boonyuen, Sivichai \& E.B.G. Jones 2010 (H)

Dictyochaeta Speg. 1923, anamorphic Ascocodinaea Samuels, Candoussau \& Magni 1997 (H)

?Glomerulispora Abdel-Wahab, AbdelAziz \& Nagahama 2010, anamorphic Torpedospora Meyers 1957 (H)

Moheitospora Abdel-Wahab, Abdel-Aziz \& Nagahama 2010, anamorphic Juncigena Kohlm., Volkm.-Kohlm \& O.E. Erikss. 1997 (H)
Mycosphere Doi 10.5943/mycosphere/3/2/5

Sporoschismopsis Hol.-Jech. \& Hennebert 1972, anamorphic Porosphaerellopsis Samuels \& E. Müll. 1982 (H)

Subclass Sordariomycetidae O.E. Erikss. \& Winka

Boliniales P.F. Cannon 2001

Boliniaceae Rick 1931

Phaeoisaria Höhn. 1909 anamorphic Lentomitella Höhn. 1906 (H)

Calosphaeriales M.E. Barr

Calosphaeriaceae Munk

Calosphaeriophora Réblová, L. Mostert, W. Gams \& Crous 2004, anamorphic Calosphaeria Tul. \& C. Tul. (H)

Lecythophora Nannf. 1934, anamorphic Sulcatistroma A.W. Ramaley 2005 (H)

Phaeocrella Réblová, L. Mostert, W. Gams \& Crous 2004, anamorphic Togniniella Réblová, L. Mostert, W. Gams Crous 2004 (H)

Phialophora-like, anamorphic Jattaea Berl. 1900 (H)

Stachybotrys-like, anamorphic Jattaea Berl. 1900 (H)

Pleurostomataceae Réblová, L. Mostert, W. Gams \& Crous

Pleurostomophora Vijaykr., L. Mostert, Jeewon, W. Gams, K.D. Hyde \& Crous 2004, anamorphic Pleurostoma Tul. \& C. Tul. 1863 (H)

Chaetosphaeriales Huhndorf, A.N. Mill. \& F.A. Fernández

Chaetosphaeriaceae Réblová, M.E. Barr \& Samuels

Cacumisporium Preuss 1851, anamorphic Chaetosphaeria Tul. \& C. Tul. 1863 (H)

Catenularia Grove 1886, anamorphic Chaetosphaeria Tul. \& C. Tul. 1863 (H)

Chalara (Corda) Rabenh. 1844, anamorphic Ascochalara Réblová 1999 and Chaetosphaeria Tul. \& C. Tul. 1863 (H)*

Chloridium Link 1809, anamorphic Chaetosphaeria Tul. \& C. Tul. 1863 and 
Melanopsammella Höhn. 1920 (H)

Codinaea Maire 1937, anamorphic Chaetosphaeria Tul. \& C. Tul. 1863 (H)

?Cordana Preuss 1851, anamorphic Porosphaerella E. Müll. \& Samuels 1982 (H)

Craspedodidymum Hol.-Jech. 1972, anamorphic Chaetosphaeria Tul. \& C. Tul. $1863(\mathbf{H})$

Cryptophiale Piroz. 1968, anamorphic Chaetosphaeria Tul. \& C. Tul. 1863 (H)

Custingophora Stolk, Hennebert \& Klopotek 1968 anamorphic Chaetosphaeria Tul. \& C. Tul. 1863 (H)

Dictyochaeta Speg. 1923, anamorphic Chaetosphaeria Tul. \& C. Tul. 1863 and Striatosphaeria Samuels \& E. Müll. 1979 (H)

Exserticlava S. Hughes 1978, anamorphic Chaetosphaeria Tul. \& C. Tul. 1863 (H)

Fusichalara S. Hughes \& Nag Raj 1973, anamorphic Chaetosphaeria Tul. \& C. Tul. 1863 (H)

Gonytrichum Nees \& T. Nees 1818, anamorphic Chaetosphaeria Tul. \& C. Tul. 1863 (H)

Harpophora W. Gams 2000, anamorphic Chaetosphaeria Tul. \& C. Tul. 1863 (H)

Hemicorynespora M.B. Ellis 1972, anamorphic Chaetosphaeria Tul. \& C. Tul. 1863 (H)

Kylindria DiCosmo, S.M. Berch \& W.B. Kendr. 1983, anamorphic Chaetosphaeria Tul. \& C. Tul. 1863 (H)

Menispora Pers. 1822, anamorphic Chaetosphaeria Tul. \& C. Tul. 1863 (H)

Monilochaetes Halst. ex Harter 1916, anamorphic Australiasca Sivan. \& Alcorn 2002 (H)*

Nawawia Marvanová 1980 (H)

Paliphora Sivan. \& B. Sutton 1985 (H)

Phaeostalagmus W. Gams 1976, anamorphic Chaetosphaeria Tul. \& C. Tul. 1863 (H)

Phialogeniculata Matsush. 1971 (H)

Phialophora-like, anamorphic Chaetosphaeria Tul. \& C. Tul. 1863 (H)

Pseudobotrytis Krzemien. \& Badura 1954, anamorphic Porosphaerella E. Müll. \&
Samuels 1982 (H)

Pyrigemmula D. Magyar \& R. Shoemaker 2011 (H)

Ramichloridium-like, anamorphic Chaetosphaerides Matsush. 2003 (H)

Rattania Prabhugaonkar \& Bhat 2009 (H)

Sporidesmium Link 1809, anamorphic Umbrinosphaeria Réblová 1999, Miyoshiella Kawam. 1929 and Lecythothecium Réblová \& Winka 2001 (H)

Sporoschisma Berk. \& Broome 1847, anamorphic Melanochaeta E. Müll., Harr \& Sulmont 1969 and Chaetosphaeria Tul. \& C. Tul. 1863 (H)

Stachybotrys Corda 1837, anamorphic Melanopsamma Niessl 1876 (H)

Thozetella Kuntze 1891 (H)

Zanclospora S. Hughes \& W.B. Kendr. 1965, anamorphic Chaetosphaeria Tul. \& C. Tul. 1863 (H)

Coniochaetales Huhndorf, A.N. Mill. \& F.A. Fernández

Coniochaetaceae Malloch \& Cain

Cladobotryum-like, anamorphic Coniochaeta (Sacc.) Cooke 1887 (H)

?Dactylaria Sacc. 1880, anamorphic Synaptospora Cain 1957 (H)

Lecythophora Nannf. 1934, anamorphic Coniochaeta (Sacc.) Cooke 1887 (H)

Phialophora-like, anamorphic Barrina A.W. Ramaley 1997 (H)

Diaporthales Nannf.

Cryphonectriaceae Gryzenh. \& M.J. Wingf. Aurapex Gryzenh. \& M.J. Wingf. 2006 (C)

Chrysoporthella Gryzenh. \& M.J. Wingf. 2004, anamorphic Chrysoporthe Gryzenh. \& M.J. Wingf. 2004 (C)

Endothiella Sacc. 1906, anamorphic Cryphonectria (Sacc.) Sacc. \& D. Sacc. 1905 (C)

Foliocryphia Cheewangkoon \& Crous 2010 (C)

Prosopidicola Crous \& C.L. Lennox 2004 (C)

Ursicollum Gryzenh. \& M.J. Wingf. 2006 (C)

Diaporthaceae Höhn. ex Wehm. Mazzantiella Höhn. 1925, anamorphic 
Mazzantia Mont. 1855 (C)

Phomopsis (Sacc.) Bubák 1905, anamorphic Diaporthe Nitschke 1870 (C)

Gnomoniaceae G. Winter

Asteroma DC. 1815, anamorphic Gnomoniella Sacc. 1881 and Plagiostoma Fuckel 1870 and Pleuroceras Riess. The type of the genus is considered to be Montagnellina stellaris (Pers.) Theiss. \& Syd. 1915 (C)

?Depazea Fr. 1818, anamorphic Pleuroceras Riess 1854 (C)

Diplodina Westend. 1857, anamorphic Plagiostoma Fuckel 1870 (C)

Discosporium Sacc. \& P. Syd. 1902 anamorphic Cryptodiaporthe Petr. 1921 C. Tul. (C)

Discula Sacc. 1884, anamorphic Apiognomonia Höhn. 1917, Gnomonia Ces. \& De Not. 1863 and Amphiporthe Petr. 1971 (C)

Gloeosporidina Petr. 1921, anamorphic Apiognomonia Höhn. 1917 (C)

Mazzantiella Höhn. 1925, anamorphic Mamianiella Höhn. 1917 (C)

Millerburtonia Cif. 1951, anamorphic Plagiostoma Fuckel 1870 (C)

Phaeocytostroma Petr. 1921, anamorphic Clypeoporthe Höhn 1919 (C)

?Septogloeum Sacc. 1880, anamorphic Pleuroceras Riess 1854 (C)

Uniseta Ciccar. 1948, anamorphic Plagiostoma Fuckel 1870 (C)

?Zythia Fr. 1849 (C)

Melanconidaceae G. Winter

Dumortieria Westend. 1857 (as Cytosporina Sacc. 1884), anamorphic Melanamphora Lafl. 1976 and Melogramma Fr. 1849 (C)

Harknessia Cooke 1881, anamorphic Wuestneia Auersw. ex Fuckel 1863 (C)

Hendersonula Speg. 1880, anamorphic Pseudovalsella Höhn. 1918 and Dictyoporthe Petr. 1955 (C)

Mastigosporella Höhn. 1914, anamorphic Wuestneiopsis J. Reid \& Dowsett 1990 (C)

Melanconiopsis Ellis \& Everh. 1900, anamorphic Massariovalsa Sacc. 1882 (C)

Melanconium Link 1809, anamorphic
Melanconis Tul. \& C. Tul. 1863 (C)

?Stilbospora Pers. 1794, anamorphic Prosthecium Fresen. 1852 (C)

Tubakia B. Sutton 1973, anamorphic Dicarpella Syd. \& P. Syd. 1921 (C)

Pseudoplagiostomaceae Cheewangkoon, M.J. Wingf. \& Crous

Cryptosporiopsis-like, anamorphic Pseudoplagiostoma Cheewangkoon, M.J. Wingf. \& Crous 2010 (C)

Pseudovalsaceae M.E. Barr

Coryneum Nees 1816, anamorphic Pseudovalsa Ces. \& De Not. 1863 (C)

Schizoparmeaceae Rossman

Coniella Höhn. 1918 (C)

Pilidiella Petr. \& Syd. 1927, anamorphic Schizoparme Shear 1923 (C)

Sydowiellaceae Lar. N. Vassiljeva

Cylindrosporella Höhn. 1916, anamorphic Stegophora Syd. \& P. Syd. 1916 (C)

Phoma-like, anamorphic Hapalocystis Auersw. ex Fuckel 1863 (C)

Togniniaceae Réblová, L. Mostert, W. Gams $\&$ Crous

Phaeoacremonium W. Gams, Crous \& M.J. Wingf. 1996, anamorphic Togninia Berl. 1900 (H)

Valsaceae Tul. \& C. Tul.

Amphicytostroma Petr. 1921, anamorphic Amphiporthe Petr. 1971 (C)

Cytospora Ehrenb. 1818, anamorphic Leucostoma (Nitschke) Höhn. 1917, Valsa Fr. 1849, Valsella Fuckel 1870 and Valseutypella Höhn. 1919 (C)

Harpostroma Höhn. 1928, anamorphic Leptosillia Höhn. 1928 (C)

?Lasmenia Speg. 1886, anamorphic Chadefaudiomyces Kamat, Rao, Patil \& Ullasa 1974 (C)

Phomopsis (Sacc.) Bubák 1905, anamorphic Clypeoporthella Petr. 1924 (C)

Diaporthales, genera incertae sedis

Craspedodidymum Hol.-Jech. 1972, anamorphic Thailandiomyces Pinruan, Sakay., K.D. Hyde \& E.B.G. Jones 
2008 (H)

Apoharknessia Crous \& S.J. Lee 2004 (C)

Apomelasmia Grove 1937 (C)

Asterosporium Kunze 1819 (C)

Craspedodidymum Hol.-Jech. 1972, anamorphic Thailandiomyces Pinruan, Sakay., K.D. Hyde \& E.B.G. Jones 2008 (H)

Cytospora-like, anamorphic Pachytrype Berl. ex M.E. Barr, J.D. Rogers \& Y.M. Ju 1993 (C)

Dwiroopa Subram. \& Muthumary

?Greeneria Scribn. \& Viala 1887 (C)

Hormonema Lagerb. \& Melin 1927, anamorphic Valsaria Ces. \& De Not. 1863 (H)

Hypodermina Höhn. 1916 (C)

Macrohilum H.J. Swart 1988 (C)

Natarajania Pratibha \& Bhat 2006 (H)

Phomopsis-like, anamorphic Caudospora Starbäck 1889 (C)

Rabenhorstia Fr. 1849, anamorphic Hercospora Fr. 1825 (C)

Sirococcus Preuss 1855 (C)

Stenocarpella Syd. \& P. Syd. 1917 (C)

Synchaetomella Decock \& Seifert 2005 (H)

Magnaporthales Thongk., Vijakr. \& K.D. Hyde

Magnaporthaceae P.F. Cannon

Clasterosporium Schwein. 1832, anamorphic Clasterosphaeria Sivan. 1984 (H)

Didymobotryum Sacc. 1886, anamorphic Ceratosphaerella Huhndorf, Greif, Mugambi \& A.N. Mill. 2008 (H)

Harpophora W. Gams 2000, anamorphic Gaeumannomyces Arx \& D.L. Olivier 1952 and Magnaporthe R.A. Krause \& R.K. Webster 1972 (H)

Mycoleptodiscus Ostaz. 1968, anamorphic Omnidemptus P.F. Cannon \& Alcorn 1994 (H)

Nakataea Hara 1939, anamorphic Magnaporthe R.A. Krause \& R.K. Webster 1972 (H)

Pyricularia Sacc. 1880, anamorphic Magnaporthe R.A. Krause \& R.K. Webster 1972 (H)

Trichocladium-like, anamorphic Gaeumannomyces Arx \& D.L. Olivier 1952 (H)
Ophiostomatales Benny \& Kimbr.

Kathistaceae Malloch \& M. Blackw.

Mattirolella S. Colla 1929 (C)

Termitaria Thaxt. 1920 (H)

Termitariopsis M. Blackw., Samson \& Kimbr. 1980 (H)

Ophiostomataceae Nannf.

Acremonium-like, anamorphic Fragosphaeria Shear 1923 (H)

Ambrosiella Brader ex Arx \& Hennebert 1965, anamorphic Grosmannia Goid. 1936 and Ophiostoma Syd. \& P. Syd. 1919 (H)

Graphium Corda 1837, anamorphic Ophiostoma Syd. \& P. Syd. 1919 (H)

Hyalobelemnospora Matsush. 1993, anamorphic Subbaromyces Hesselt. 1953 (H)

Hyalorhinocladiella H.P. Upadhyay \& W.B. Kendr. 1975, anamorphic Ceratocystiopsis H.P. Upadhyay \& W.B. Kendr. 1975, Klasterskya Petr. 1940 and Ophiostoma Syd. \& P. Syd. 1919 (H)

Hyalorhinocladiella-like, anamorphic Klasterskya Petr. 1940 (H)

Leptographium Lagerb. \& Melin 1927, anamorphic Grosmannia Gold. 1936 (H)*

Pesotum J.L. Crane \& Schokn. 1973, anamorphic Ophiostoma Syd. \& P. Syd. 1919 (H)

Pesotum-like, anamorphic Grosmannia Gold. 1936 (H)

Phialographium H.P. Upadhyay \& W.B. Kendr. 1974, anamorphic Ophiostoma Syd. \& P. Syd. 1919 (C)

Raffaelea Arx \& Hennebert 1965, anamorphic Grosmannia Goid. 1936 and Ophiostoma Syd. \& P. Syd. 1919 (H)

Sporothrix Hektoen \& C.F. Perkins 1901, anamorphic Ophiostoma Syd. \& P. Syd. $1919(\mathbf{H})$

Sordariales Chad. ex D. Hawksw. \& O.E. Erikss.

Chaetomiaceae G. Winter Acrophialophora Edward 1961 (H)

Botryotrichum Sacc. \& Marchal 1885, anamorphic Chaetomium Kunze 1817 and Farrowia D. Hawksw. (H) 
Humicola Traaen 1914, anamorphic Chaetomium Kunze 1817 (H)

Madurella Brumpt 1905 (H)

Myceliophthora Costantin 1892, anamorphic Corynascus Arx 1973 and Thielavia Zopf 1876 (H)

Papulaspora-like, Preuss 1851, anamorphic Chaetomium Kunze 1817 (H)

Phialophora-like, anamorphic Chaetomidium (Zopf) Sacc. 1882 (H)

Retroconis de Hoog \& Bat. Vegte 1989 (H)

Taifanglania Z.Q. Liang, Y.F. Han, H.L. Chu \& R.T.V. Fox 2009, anamorphic Thielavia Zopf 1876 (H)

Trichocladium Harz 1871, anamorphic Chaetomium Kunze 1817 (H)

Trichosporiella Kamyschko 1960, anamorphic Thielavia Zopf 1876 (H)

Lasiosphaeriaceae Nannf.

Angulimaya Subram. \& Lodha 1964, anamorphic Bombardioidea C. Moreau ex N. Lundqv. 1972 (H)

Bagadiella Cheewangkoon \& Crous 2009 (H)

Cladorrhinum Sacc. \& Marchal 1885, anamorphic Apiosordaria Arx \& W. Gams 1967, Apodospora Cain \& J.H. Mirza 1970, Arnium Nitschke ex G. Winter 1873, Cercophora Fuckel 1870 and Podospora Ces. 1856 (H)

Mammaria Ces. ex Rabenh. 1854, anamorphic Pseudocercophora Subram. \& Sekar 1986 and Cercophora Fuckel 1870 (H)

Phialophora-like, anamorphic Cercophora Fuckel 1870, Eosphaeria Höhn. 1917, Lasiosphaeria Ces. \& De Not. 1863 and Podospora Ces. 1856 (H)

Sordariaceae G. Winter

Chrysonilia Arx 1981, anamorphic Neurospora Shear \& B.O. Dodge 1927 (H)

Trichosporiella Kamyschko 1960, anamorphic Diplogelasinospora Cain 1961 (H)

Virgariella S. Hughes 1953, anamorphic Effetia Bartoli, Maggi \& Persiani 1984 (H)

Sordariales, genera incertae sedis

Kionochaeta P.M. Kirk \& B. Sutton 1986
(H)

Lasiadelphia Réblová \& W. Gams 2011 (H)

Lunulospora Ingold 1942 (H)

Madurella Brumpt 1905 (H)

Monodictys S. Hughes 1958 anamorphic Ascotaiwania Sivan. \& H.S. Chang 1992 (H)

Monotosporella S. Hughes 1958, anamorphic Ascotaiwania Sivan. \& H.S. Chang 1992 (H)

Papulospora Preuss 1851, anamorphic Sordaria Ces. \& De Not. 1863 (H)

Ramophialophora M. Calduch, Stchigel, Gené \& Guarro 2004 (H)

Trichocladium-like, anamorphic Ascolacicola Ranghoo \& K.D. Hyde 1998, Ascotaiwania Sivan. \& H.S. Chang 1992 (H)

Ypsilonia Lév. 1846, anamorphic Acanthotheciella Höhn. 1911 (H)

Sordariomycetidae, families incertae sedis

Amplistromataceae Huhndorf, A.N. Mill., M. Greif \& Samuels

Acrodontium-like, anamorphic Amplistroma Huhndorf, A.N. Mill., M. Greif \& Samuels 2009 (H)

Pseudogliomastix W. Gams 1985, anamorphic Wallrothiella Sacc. 1882 (H)

Annulatascaceae S.W. Wong, K.D. Hyde \& E.B.G. Jones

Phaeoisaria Höhn. 1909, anamorphic Rhamphoria Niessl 1876 (H)

Phialophora-like, anamorphic Teracosphaeria Réblová \& Seifert 2007 (H)

Cephalothecaceae Höhn.

Acremonium-like, anamorphic Albertiniella Kirschst. 1936 (H)

Chalara (Corda) Rabenh. 1844, anamorphic Cryptendoxyla Malloch \& Cain 1970 (H)*

Phialemonium W. Gams \& McGinnis 1983 (H)

Tritirachium Limber 1940, anamorphic Cephalotheca Fuckel 1871 (H)

Helminthosphaeriaceae Samuels, Candoussau \& Magní

Diplococcium Grove 1885, anamorphic 
Helminthosphaeria Fuckel 1870 (H)

Endophragmiella B. Sutton 1973, anamorphic Echinosphaeria A.N. Mill. \& Huhndorf 2004 (H)

Selenosporella-like, anamorphic Ruzenia O. Hilber $2002(\mathbf{H})$

Spadicoides S. Hughes 1958, anamorphic Tengiomyces Réblová 1999 (H)

Vermiculariopsiella Bender 1932, anamorphic Echinosphaeria A.N. Mill. \& Huhndorf 2004 (H)

Sordariomycetidae, genera incertae sedis

Sterigmatobotrys Oudem. 1886 (H)*

Brachysporiella Bat. 1952, anamorphic Ascotaiwania Sivan. \& H.S. Chang 1992 (H)

Conioscypha Höhn. 1904, anamorphic Conioscyphascus Réblová \& Seifert 2004 (H)

Endophragmiella B. Sutton 1973, anamorphic Phaeotrichosphaeria Sivan. 1983 (H)

Menisporopsis S. Hughes 1952, anamorphic Menisporopascus Matsush. 2003 (H)

Phialophora-like, anamorphic Linocarpon Syd. \& P. Syd. 1917 (H)

Pleurothecium Höhn. 1919, anamorphic Carpoligna F.A. Fernández \& Huhndorf 1999 (H)

Ramichloridium-like, anamorphic Barbatosphaeria Reblova 2007 (H)

Rhodoveronaea Arzanlou, W. Gams \& Crous 2007 (H)

Spadicoides S. Hughes 1958 (H)

Sporothrix-like, anamorphic Barbatosphaeria Reblova 2008 (H)

Subclass Xylariomycetidae sensu O.E. Erikss. \& Winka

Xylariales Nannf.

Amphisphaeriaceae G. Winter

Bartalinia Tassi 1900 (C)

?Beltraniella Subram. 1952, anamorphic Leiosphaerella Höhn. 1919 (H)

Bleptosporium Steyaert 1961, anamorphic Amphisphaeria Ces. \& De Not. 1863 (C)

Ceratosporium Schwein. 1832, anamorphic Iodosphaeria Samuels, E. Müll. \& O. Petrini 1987 (H)
?Chaetoconis Clem. 1909, anamorphic Ceriospora Niessl 1876 (C)

Discosia Lib. 1837, anamorphic Adisciso Kaz. Tanaka, Okane \& Hosoya 2011 (C)*

Doliomyces Steyaert 1961 (C)

?Hyalotiopsis Punith. 1970, anamorphic Ellurema Nag Raj \& W.B. Kendr. 1986 (C)

Immersidiscosia Kaz. Tanaka, Okane \& Hosoya 2011 (C)*

Labridella Brenckle 1929, anamorphic Griphosphaerioma Höhn. 1918 (C)

Labridium Vestergr. 1897, anamorphic Discostroma Clem. 1909 (C)

Microdochium Syd. 1924, anamorphic Monographella Petr. 1924 (H)

Monochaetia (Sacc.) Allesch. 1902 (C)

Monochaetinula Muthumary, Abbas \& B. Sutton 1986 (C)

Monochaetiopsis L. Cai, Jeewon \& K.D. Hyde 2003, anamorphic Dyrithiopsis L. Cai, R. Jeewon \& K.D. Hyde 2003 (C)

Morinia Berl. \& Bres. 1889 (C)

?Pestalotia De Not. 1841, anamorphic Broomella Sacc. 1883 (C)

Pestalotiopsis Steyaert 1949, anamorphic Neobroomella Petr. 1947 and Pestalosphaeria M.E. Barr 1975 (C)

Phlogicylindrium Crous, Summerb. \& Summerell 2006 (H)

Sarcostroma Cooke 1871, anamorphic Griphosphaerioma Höhn. 1918 (C)

Seimatosporium Corda 1833, anamorphic anamorphic Discostroma Clem. 1909 (C)

Seiridium Nees 1816, anamorphic Blogiascospora Shoemaker, E. Müll. \& Morgan-Jones 1966 and Lepteutypa Petr. 1923 (C)

Selenosporella G. Arnaud ex MacGarvie 1969, anamorphic Oxydothis Penz. \& Sacc. 1898 and Iodosphaeria Samuels, E. Müll. \& O. Petrini 1987 (H)

Sporocadus Corda 1839 (C)

Synnemapestaloides T. Handa \& Y. Harada 2004 (H)

?Truncatella Steyaert 1949 anamorphic Broomella Sacc. 1883 (C)

Vermisporium H.J. Swart \& M.A. Will. 1983 (C)

Zetiasplozna Nag Raj 1993 (C) 
Diatrypaceae Nitschke

Dumortieria Westend. 1857 (some as Cytosporina Sacc. 1884), anamorphic Cryptosphaeria Ces. \& De Not. 1863, Diatrype Fr. 1849, Eutypa Tul. \& C. Tul. 1863 and Eutypella (Nitschke) Sacc. 1875 (C)

Libertella Desm. 1830, anamorphic Diatrype Fr. 1849, Diatrypella (Ces. \& De. Not.) De. Not. 1863, Eutypa Tul. \& C. Tul. 1863 and Eutypella (Nitschke) Sacc. 1875 (C)

Diatrypaceae Nitschke 1869

Phaeoisaria Höhn. 1909 anamorphic Eutypella (Nitschke) Sacc. 1875 (H)

\section{Hyponectriaceae Petr.}

Arthrinium Kunze 1817, anamorphic Physalospora Niessl (H)

Aureobasidium Viala \& G. Boyer 1891, anamorphic Discosphaerina Höhn. 1917 (H)

Beltraniella Subram. 1952, anamorphic Pseudomassaria Jacz. 1894 (H)

Hormonema-like, anamorphic Discosphaerina Hohn 1917 (H)

Kabatia Bubák 1904, anamorphic Discosphaerina Höhn. 1917 (C)

Kabatiella Bubák 1907, anamorphic Discosphaerina Höhn. 1917 (H)

Sarcophoma Höhn. 1916, anamorphic Discosphaerina Hohn 1917 (C)

Selenophoma Maire 1907, anamorphic Discosphaerina Hohn 1917 (C)

Graphostromataceae M.E. Barr, J.D. Rogers \& Y.M. Ju

Nodulisporium-like, anamorphic Graphostroma Piroz. 1974 (H)

Xylariaceae Tul. \& C. Tul.

Acanthodochium Samuels, J.D. Rogers \& Nagas., anamorphic Astrocystis Berk. \& Broome and Collodiscula I. Hino \& Katum. 1955 (H)

Arthroxylaria Seifert \& W. Gams 2002

Dematophora R. Hartig 1883, anamorphic Rosellinia De Not. 1844 (H)

?Dicyma Boulanger 1897, anamorphic Ascotricha Berk. 1838 (H)
Geniculisynnema Okane \& Nakagiri 2007, anamorphic Nemania Gray 1821 (H)

Geniculosporium Chesters \& Greenh. 1964, anamorphic Entoleuca Syd. 1922, Leprieuria Laessøe, J.D. Rogers \& Whalley 1989, Nemania Gray 1821, Phylacia Lév. 1845, Podosordaria Ellis \& Holw. 1897, Rosellinia De Not. 1844 and Xylaria Hill ex Schrank 1789 (H)

Geniculosporium-like, anamorphic Halorosellinia Whalley, E.B.G. Jones, K.D. Hyde \& Læssøe 2000, Jumillera J.D. Rogers, Y.M. Ju \& San Martín 1997 and Kretzschmaria Fr. 1849 (H)

Hypocreodendron Henn. 1897, anamorphic Discoxylaria J.C. Lindq. \& J.E. Wright 1964 (H)

Libertella Desm. 1830 (C)

Libertella-like, anamorphic Barrmaelia Rappaz 1995, Creosphaeria Theiss. 1910, Jumillera J.D. Rogers, Y.M. Ju \& San Martín 1997 and Lopadostoma (Nitschke) Traverso 1906 (C)

Lindquistia Subram. \& Chandrash. 1977, anamorphic Poronia Willd. 1787 (H)

Masoniomyces J.L. Crane \& Dumont 1975, anamorphic Camillea Fr. 1849 (H)

Mirandina-like, anamorphic Kretzschmariella Viégas 1944 (H)

Moelleroclavus Henn. 1902, anamorphic Xylaria Hill ex Schrank 1789 (H)

Muscodor Worapong, Strobel \& W.M. Hess 2001 (H)

Nodulisporium Preuss 1849, anamorphic Annulohypoxylon Y.M. Ju, J.D. Rogers \& H.M. Hsieh 2005, Biscogniauxia Kuntze 1891, Daldinia Ces. \& De Not. 1863, Entonaema Möller 1901, Hypoxylon Bull. 1791, Induratia Samuels, E. Müll. \& O. Petrini, Pyrenomyxa Morgan 1895 (as Pulveria Malloch \& Rogerson 1977), Thamnomyces Ehrenb. 1820, Theissenia Maubl. 1914, Thuemenella Penz. \& Sacc. 1898 and Xylaria Hill ex Schrank (H)

Nodulosporium-like, anamorphic Rhopalostroma D. Hawksw. 1977 and Ruwenzoria J. Fornier, M. Stadler, Læssøe \& C. Decock 2010 (H)

Padixonia Subram. 1972, anamorphic Xylaria Hill ex Schrank (H) 
Rhinocladiella-like, anamorphic Obolarina Pouzar 1986 (H)

Sporothrix-like, anamorphic Rostrohypoxylon J. Fourn. \& M. Stadler 2010 and Ruwenzoria J. Fornier, M. Stadler, Læssøe \& C. Decock 2010 (H)

Triplicaria P. Karst. 1889, anamorphic Hypoxylon Bull. 1791 (H)

Virgaria Nees 1816, anamorphic Ascovirgaria J.D. Rogers \& Y.M. Ju 2002 (H)

Virgariella-like, anamorphic Hypoxylon Bull. 1791 (H)

Vivantia J.D. Rogers, Y.M. Ju \& Cand. 1996 (H)

Xylocladium P. Syd. ex Lindau 1900, anamorphic Camillea Fr. 1849 (H)

Xylocoremium J.D. Rogers 1984, anamorphic Xylaria Hill ex Schrank 1789 (H)

Xylariales, genera incertae sedis

Chaetochalara B. Sutton \& Piroz. 1965 (H)

Chalara (Corda) Rabenh. 1844 (H)*

Hadrotrichum Fuckel 1865 (H)

Humicola-like, anamorphic Ascotrichella Valldos. \& Guarro 1988 (H)

Idriella P.E. Nelson \& S. Wilh. 1956, anamorphic Phomatospora Sacc. 1875 (H)

Melanographium Sacc. 1913, possibly anamorphic Lasiobertia Sivan. 1978 (H)

Nodulisporium-like, anamorphic Coniolariella D. Garciá, Stchigel \& Guarro $(\mathbf{H})$

Rhinocladiella-like, anamorphic Coniolariella D. Garciá, Stchigel \& Guarro (H)

Selenosporella G. Arnaud ex MacGarvie 1969, anamorphic Diatrypasimilis J. Zhou, \& Kohlm. 2010 (H)

Sporidesmina Subram. \& Bhat 1989 (H)

Subramaniomyces Varghese \& V.G. Rao 1980 (H)

Sordariomycetes, orders incertae sedis

Lulworthiales Kohlm., Spatafora \& Volkm.Kohlm.

Lulworthiaceae Kohlm., Spatafora \& Volkm.Kohlm.

Anguillospora Ingold 1942, anamorphic Lindra I.M. Wilson 1956 (H)

Cumulospora I. Schmidt 1985, anamorphic Lulwoana Kohlm., Volkm.-
Kohlm., J. Campb., Spatafora \& Gräfenhan 2005 and Lulworthia G.K. Sutherl. 1916 (H)

Halazoon Abdel-Aziz, Abdel-Wahab \& Nagahama 2010, anamorphic Lulworthia G.K. Sutherl. 1916 (C)

Hydea K.L. Pang \& E.B.G. Jones 2010 (H)

Matsusporium E.B.G. Jones \& K.L. Pang 2010 (H)

Moleospora Abdel-Wahab, Abdel-Aziz \& Nagahama 2010 (H)

Moromyces Abdel-Wahab, K.L. Pang, Nagahama, Abdel-Aziz \& E.B.G. Jones 2010 (H)

Zalerion R.T. Moore \& Meyers 1962, anamorphic Lulwoana Kohlm., Volkm.Kohlm, J. Campb., Spatafora \& Gräfenhan 2005 (H)

Lulworthiales, genera incertae sedis

Halazoön Abdel-Aziz, Abdel Wahab \& Nagah. 2011 (H)

Phyllachorales M.E. Barr

Phyllachoraceae Theiss. \& H. Syd.

Acerviclypeatus Hanlin 1990, anamorphic Ophiodothella Boyd 1934 (C)

Baeumleria Petr. \& Syd. 1927, anamorphic Phyllachora Nitschke ex Fuckel 1870 (as Trabutia Sacc. \& Roum. 1881) (C)

Colletogloeum-like, anamorphic Coccodiella Hara 1909 (C)

Cyclodomus Höhn. 1909, anamorphic Maculatifrons K.D. Hyde 1996 (C)

Diachorella Höhn. 1918, anamorphic Diachora Müll. 1893 (C)

Hysterodiscula Petr. 1942, anamorphic Orphnodactylis Malloch \& A. Mallik 1998 (C)

Linochora Höhn. 1910, anamorphic Phyllachora Nitschke ex Fuckel 1870 (C)

Mycohypallage B. Sutton 1963, anamorphic Deshpandiella Kamat \& Ullasa 1973 (C)

Oswaldina Rangel 1921, anamorphic Apiosphaeria Höhn. 1909 (C)

Polystigmina Sacc. 1884, anamorphic Polystigma DC. 1815 (C)

Pseudothiopsella Petr. 1928, anamorphic Pseudothiella Petr. 1928 (C) 
Rhodosticta Woron. 1911, anamorphic Polystigma DC. 1815 and Stigmatula (Sacc.) Syd. \& P. Syd. 1901 (C)

Trichosphaeriales M.E. Barr 1983

Trichosphaeriaceae G. Winter 1885

Brachysporium Sacc. 1886, anamorphic Cryptadelphia Réblová \& Seifert 2004 (H)

Koorchaloma Subram. 1953, anamorphic Kananascus Nag Raj 1984 (H)

Stanjehughesia Subram. 1992, anamorphic Miyoshiella Kawam. 1929 and Umbrinosphaeria Réblová 1999 (H)

Stromatographium Höhn. 1907, anamorphic Fluviostroma Samuels \& E. Müll. 1980 (H)

Sordariomycetes, family incertae sedis

Apiosporaceae K.D. Hyde, J. Fröhlich, Joanne

E. Taylor \& M.E. Barr

Arthrinium Kunze 1817, anamorphic Apiospora Sacc. $1875(\mathbf{H})$

Cordella Speg. 1886, anamorphic Apiospora Sacc. 1875 (H)

Pteroconium Sacc. ex Grove 1914, anamorphic Apiospora Sacc. 1875 (H)

Scyphospora L.A. Kantsch. 1928, anamorphic Apiospora Sacc. 1875 (C)

Thyridiaceae O.E. Erikss. \& J.Z. Yue

Pleurocytospora Petr. 1923, anamorphic Thyridium Nitschke 1867 (C)

Sordariomycetes, genera incertae sedis

Cryptomycella Höhn. 1925, anamorphic Cryptomycina Höhn. 1977 (C)

?Custingophora Stolk, Hennebert \& Klopotek 1968 (H)

Didymostilbe-like, anamorphic Ornatispora K.D. Hyde, Goh, Joanne E. Taylor, J. Fröhl. 1999 (H)

Dinemasporium Lév. 1846, anamorphic Phomatospora Sacc. 1875 (C)

Ellisembia Subram. 1992 (H)

Linkosia A. Hern. Gut. \& B. Sutton 1997 (H)

Myrmecridium Arzanlou, W. Gams \& Crous 2007 (H)

Nigrospora Zimm. 1902, anamorphic Khuskia H.J. Huds. 1963 (H)

Penicillifer Emden 1968, anamorphic
Mycosphere Doi 10.5943/mycosphere/3/2/5

Stellosetifera Matsush.1996 (H)

Phomatosporella Tak. Kobay. \& K. Sasaki 1982, anamorphic Phomatospora Sacc. 1875 (C)

Ramichloridium-like, anamorphic Tectonidula Réblová 2009 (H)

Sporothrix-like, anamorphic Phomatospora Sacc. 1875 and Tectonidula Réblová 2009 (H)

Subphylum PEZIZOMYCOTINA, orders incertae sedis

Lahmiales O. E. Erikss.

Lahmiaceae O. E. Erikss.

Cyphellophora G.A. de Vries 1962, anamorphic Lahmia Körb. 1861 (H)

ASCOMYCOTA, families incertae sedis

Families and genera that cannot be placed in any of the classes and orders accepted in the present classification with a high degree of probability are listed below.

Amorphothecaceae Parbery

Hormoconis Arx \& G.A. de Vries 1973, anamorphic Amorphotheca Parbery 1969 (H)

Batistiaceae Samuels \& K.F. Rodrigues

Acrostroma Seifert 1987, anamorphic Batistia Cif. 1958 (H)

Pseudeurotiaceae Malloch \& Cain

Teberdinia Sogonov, W. Gams, Summerb. \& Schroers 2005, anamorphic Pseudeurotium J.F.H. Beyma 1937 (H)

Seuratiaceae Vuill. ex M.E. Barr

Atichia Flot. 1850, anamorphic Seuratia Pat. 1904 (H)

ASCOMYCOTA, genera incertae sedis

Abropelta B. Sutton 1986 (C)

Acarellina Bat. \& H. Maia 1960 (C)

Acaroconium Kocourk. \& D. Hawksw. 2008 (C)

Acarocybe Syd. 1937 (H)

Acarocybella M.B. Ellis 1960 (H)

Acarocybellina Subram. 1992 (H)

Acarocybiopsis J. Mena, A. Hern. Gut. \&

Mercado 1999 (H)

Acaropeltis Petr. 1937 (C) 
Achoropeltis Syd. 1929 (C)

Aciculariella G. Arnaud 1954 (H)

Acontium Morgan 1902 (H)

Acremoniula G. Arnaud 1954 (H)

Acrodictyella W.A. Baker \& Partr. 2001

(H)

Acrodictyopsis P.M. Kirk 1983 (H)

Acrodictys M.B. Ellis 1961 (H)

Acrodontiella U. Braun \& Scheuer 1995

(H)

Acrophragmis Kiffer \& Reisinger 1970

(H)

Acrostaurus Deighton \& Piroz. 1972 (H)

Actinocladium Ehrenb. 1819 (H)

Actinotexis Arx 1960 (C)

Actinothecium Ces. 1854 (H)

Actinothyrium Kunze 1823 (C)

Acumispora Matsush. 1980 (H)

Aegeritella Bałazy \& J. Wiśn. 1974 (H)

Aenigmatospora R.F. Castañeda, Saikawa,

Guarro \& Calduch 1999 (?H)

Agaricodochium X.J. Liu 1981 (H)

Agarwalomyces R.K. Verma \& Kamal 1987 (H)

Agrabeeja Subram. 1995 (H)

Agyriella Sacc. 1884 (H)

Agyriellopsis Höhn. 1903 (C)

Ahmadia Syd. 1939 (C)

Ajrekarella Kamat \& Kalani 1964 (C)

Alatosessilispora K. Ando \& Tubaki 1984 (H)

Alciphila Harmaja 2002 (H)

Algonquinia R.F. Castañeda \& W.B.

Kendr. 1991 (H)

Alloneottiosporina Nag Raj 1993 (C)

Allothyriella Bat., Cif. \& Nascim. 1959

(C)

Allothyrina Bat. \& J.L. Bezerra 1964 (C)

Allothyriopsis Bat., Cif. \& H. Maia 1959

(C)

Alpakesa Subram. \& K. Ramakr. 1954 (C)

Alpakesiopsis Abbas, B. Sutton, Ghaffar \&

A. Abbas 2003 (C)

Alveophoma Alcalde 1952 (C)

Alysidiopsis B. Sutton 1973 (H)

Amallospora Penz. 1897 (H)

Amblyosporium Fresen. 1863 (H)

Amerodiscosiella M.L. Farr 1961 (C)

Amerodiscosiellina Bat. \& Cavalc. 1966

(C)

Amerosporiopsis Petr. 1941 (C)
Mycosphere Doi 10.5943/mycosphere/3/2/5

Amerosympodula Matsush. 1996 (H)

Amoenodochium Peláez \& R.F. Castañeda 1996 (H)

Amoenomyces R.F. Castañeda, Saikawa \& Hennebert 1996 (H)

Amphichaetella Höhn. 1916 (H)

Amphophialis R.F. Castañeda, W.B.

Kendr. \& Guarro 1998 (H)

Amphoropycnium Bat. 1963 (C)

Ampullicephala R.F. Castañeda, Minter \& M. Stadler 2009 (H)

Ampulliferina B. Sutton 1969 (H)

Anaphysmene Bubák 1906 (C)

Anarhyma M.H. Pei \& Z.W. Yuan 1986 (C)

Anaselenosporella Heredia, R.F. Castañeda \& R.M. Arias 2010 (H)

Ancoraspora Mig. Rodr. 1982 (H)

Ancorasporella J. Mena, Mercado \& Heredia 1998 (H)

Angiopomopsis Höhn. 1912 (C)

Angulospora Sv. Nilsson 1962 (H)

Annellodentimyces Matsush. 1985 (H)

Annellodochium Deighton 1969 (H)

Annellolacinia B. Sutton 1964 (C)

Annellophora S. Hughes 1952 (H)

Annellophorella Subram. 1962 (H)

Annellophragmia Subram. 1963 (H)

Annellosympodia McTaggart, R.G. Shivas

\& U. Braun 2007 (H)

Antennopsis R. Heim 1952 (H)

Anthopsis Fil. March., A. Fontana \& Luppi

Mosca 1977 (H)

Anthracoderma Speg. 1888 (C)

Antromyces Fresen. 1850 (H)

Anulohypha Cif. 1962 (H)

Anungitea B. Sutton 1973 (H)

Anungitopsis R.F. Castañeda \& W.B.

Kendr. 1990 (H)

Aoria Cif. 1962 (C)

Aphanofalx B. Sutton 1986 (C)

Apiocarpella Syd. \& P. Syd. 1919 (C)

Apogloeum Petr. 1954 (C)

Aporellula B. Sutton 1986 (C)

Aposporella Thaxt. 1920 (H)

Arachnophora Hennebert 1963 (H)

Arachnospora R.F. Castañeda, Minter \& Camino 2003 (H)

Arborillus Munt.-Cvetk. \& Gómez-Bolea 1998 (H)

Arborispora K. Ando 1986 (H)

Arbusculina Marvanová \& Descals 1987 
(H)

Arcuadendron Sigler \& J.W. Carmich. 1976 (H)

Ardhachandra Subram. \& Sudha 1978 (H)

Argopericonia B. Sutton \& Pascoe 1987

(H)

Aristastoma Tehon 1933 (C)

Arthrobotryum Ces. 1854 (C)

Arthrocladium Papendorf 1969 (H)

Arthrocristula Sigler, M.T. Dunn \& J.W.

Carmich. 1982 (H)

Arthrosporium Sacc. 1880 (H)

Arthrowallemia R.F. Castañeda, Dania

García \& Guarro 1998 (H)

Articulophora C.J.K. Wang \& B. Sutton 1982 (H)

Artocarpomyces Subram. 1996 (H)

Arxiella Papendorf 1967 (H)

Asbolisiomyces Bat. \& H. Maia 1961 (C)

Ascochytopsis Henn. 1905 (C)

Ascochytulina Petr. 1922 (C)

Ascospora Mont. 1849 (C)

Ashtaangam Subram. 1995 (H)

Aspilaima Bat. \& H. Maia 1961 (C)

Astelechia Cif. 1962 (H)

Asterinothyriella Bat. \& Cif. 1959 (C)

Asterinothyrium Bat., Cif. \& H. Maia 1959

(C)

Asteroconium Syd. \& P. Syd. 1903 (C)

Asteromyces F. Moreau \& V. Moreau, in

Hennenert $1962(\mathbf{H})$

Asterostomopora Bat. \& H. Maia 1960 (C)

Asterostomopsis Bat., Cif. \& H. Maia 1959

(C)

Asterostomulina Bat., J.L. Bezerra \& H. Maia 1964 (C)

Astronatelia Bat. \& H. Maia 1962 (C)

Atractilina Dearn. \& Barthol. 1924 (H)

Atrosetiphiale Matsush. 1995 (H)

Aurantiosacculus Dyko \& B. Sutton 1979

(C)

Aurosphaeria Sun J. Lee, Strobel, Eisenman, Geary, Vargas \& S.A. Strobel 2009 (C)

Avesicladiella W.P. Wu, B. Sutton \& Gange 1997 (H)

Avettaea Petr. \& Syd. 1927 (C)

Bachmanniomyces D. Hawksw. 1981 (C)

Bacillopeltis Bat. 1957 (C)

Bactridiopsis Henn. 1904 (H)

Bactridium Kunze 1817 (H)

Bactrodesmiastrum Hol.-Jech 1984 (H)
Mycosphere Doi 10.5943/mycosphere/3/2/5

Bactrodesmiella M.B. Ellis 1959 (H)

Badarisama Kunwar, J.B. Manandhar \& J.B. Sinclair 1986 (H)

Bahuchashaka Subram. 1978 (H)

Bahugada K.A. Reddy \& Vasant Rao 1984 (H)

Bahukalasa Subram. \& Chandrash. 1979 (H)

Bahusandhika Subram. 1956 (H)

Balaniopsis P.M. Kirk 1985 (H)

Balanium Wallr. 1833 (H)

Barbarosporina Kirulis 1942 (C)

Barnettella D. Rao \& P.Rag. Rao 1964 (C)

Basauxia Subram. 1995 (H)

Batistina Peres 1961 (C)

Beauveriphora Matsush 1975 (H)

Beccopycnidium F. Stevens 1930 (C)

Beejadwaya Subram. 1978 (H)

Belemnospora P.M. Kirk 1981 (H)

Bellulicauda B. Sutton 1967 (C)

Beltrania Penz. 1882 (H)

Beltraniomyces Monohar, D.K. Agarwal \& Rao 2003 (H)

Beltraniopsis Bat. \& J.L. Bezerra 1960 (H)

Beniowskia Racib. 1900 (H)

Benjaminia S. Ahmad 1967 (C)

Benjpalia Subram. \& Bhat 1989 (H)

Bharatheeya D'Souza \& Bhat 2002 (H)

Bhatia W.A. Baker \& Morgan-Jones 2009

(H)

Bibanasiella R.F. Castañeda \& W.B.

Kendr. 1991 (H)

Biflagellospora Matsush. 1975 (H)

Biflagellosporella Matsush. 1993 (H)

Bimeris Petr. 1949 (C)

Bioconiosporium Bat. \& J.L. Bezerra 1964 (H)

Biophomopsis Petr. 1931 (C)

Bispora Corda 1837 (H)

Bisseomyces R.F. Castañeda 1985 (H)

Blarneya D. Hawksw., Coppins \& P. James $1980(\mathbf{H})$

Blastacervulus H.J. Swart 1988 (C)

Blastocatena Subram. \& Bhat 1989 (H)

Blastodictys M.B. Ellis 1976 (H)

Blastofusarioides Matsush. 1996 (H)

Blastophorella Boedijn 1937 (H)

Blastophorum Matsush. 1971 (H)

Blastophragma Subram. 1995 (H)

Blennoria Moug. \& Fr. 1825 (C)

Blennoriopsis Petr. 1919 (C)

Blodgettia Harv. 1858 (H) 
Bomplandiella Speg. 1886 (H)

Bostrichonema Ces. 1867 (H)

Botryoderma Papendorf \& H.P. Upadhyay

1969 (H)

Botryodiplodina Dias \& Sousa da Câmara 1954 (C)

Botryomonilia Goos \& Piroz. 1975 (H)

Botryosporium Corda 1831 (H)

Brachydesmiella G. Arnaud ex S. Hughes 1961 (H)

Brachysporiellina Subram. \& Bhat 1989 (H)

Brachysporiopsis Yanna, W.H. Ho \& K.D. Hyde 2004 (H)

Brefeldiopycnis Petr. \& Cif. 1932 (H)

Brevicatenospora R.F. Castañeda, Minter \& Saikawa $2006(\mathbf{H})$

Briosia Cavara 1888 (H)

Brycekendrickia Nag Raj 1973 (C)

Bulbilopycnis Matsush. 1996 (C)

Bulbocatenospora R.F. Castañeda \& Iturr. 2000 (H)

Bullaserpens Bat., J.L. Bezerra \& Cavalc. 1965 (C)

Calcarispora Marvanová \& Marvan 1963 (H)

Calcarisporiella de Hoog 1974 (H)

Calceispora Matsush. 1975 (H)

Callistospora Petr. 1955 (C)

Calocline Syd. 1939 (C)

?Calongia D. Hawksw. \& Etayo 2010 (H)

Camarographium Bubák 1916 (C)

Camaropycnis E.K. Cash 1945 (C)

Camarosporellum Tassi 1902 (C)

Camarosporiopsis Abbas, B. Sutton \& Ghaffar 2000 (C)

Camposporidium Nawawi \& Kuthub. 1988 (H)

Camptomeris Syd. 1927 (H)

Campylospora Ranzoni 1953 (H)

Candelabrum Beverw. 1951 (H)

Candelosynnema K.D. Hyde \& Seifert 1992 (H)

Capitorostrum Bat. 1957 (C)

Capsicumyces Gamundí, Aramb. \& Giaiotti 1979 (H)

Carrismyces R.F. Castañeda \& Heredia $2000(\mathbf{H})$

Castanedaea W.A. Baker \& Partr. 2001 (H)

Catenella Bat. \& Peres 1963 (C)
Catenocuneiphora Matsush. 2003 (H)

Catenophora Luttr. 1940 (C)

Catenophoropsis Nag Raj \& W.B. Kendr. 1988 (C)

Catenospegazzinia Subram. 1991 (H)

Catenosubulispora Matsush. 1971 (H)

Catenosynnema Kodsueb, K.D. Hyde \& W.H. Ho 2007 (H)

Catenulaster Bat. \& C.A.A. Costa 1959 (C)

Catinopeltis Bat. \& C.A.A. Costa 1957 (C)

Catinula Lév. 1848 (C)

Ceeveesubramaniomyces Pratibha, K.D. Hyde \& Bhat 2005 (H)

Ceratocladium Corda 1839 (H)

Ceratophorum Sacc. 1880 (H)

Ceratopycnis Höhn. 1915 (C)

Ceratosporella Höhn. 1923 (H)

Cercosperma G. Arnaud ex B. Sutton \& Hodges 1983 (H)

Ceuthosira Petr. 1924 (C)

Ceuthosporella Petr. \& Syd. 1923 (C)

Chaetendophragmia Matsush. 1971 (H)

Chaetoblastophorum Morgan-Jones 1977 (H)

Chaetocytostroma Petr. 1920 (C)

Chaetodiplis Clem. 1931 (C)

Chaetodiplodia P. Karst. 1884 (C)

Chaetodiplodina Speg. 1910(C)

Chaetopeltaster Katum. 1975 (C)

Chaetophiophoma Speg. 1910 (C)

Chaetophoma Cooke 1878 (C)

Chaetopsis Grev. 1825 (H)

Chaetopyrena Pass. 1881 (C)

Chaetoseptoria Tehon 1937 (C)

Chalarodendron C.J.K. Wang \& B. Sutton 1984 (H)

Chalarodes McKenzie 1991 (H)

Chantransiopsis Thaxt. 1914 (H)

Characonidia Bat. \& Cavalc. 1965 (C)

Charomyces Seifert 1987 (H)

Chasakopama Manohar., Bagyan., N.K. Rao \& Kunwar 2009 (H)

Cheilaria Lib. 1830 (C)

Cheiroidea W.A. Baker \& Morgan-Jones 2009 (H)

Cheiromyceopsis Mercado \& J. Mena 1988 (H)

Cheiromyces Berk. \& M.A. Curtis 1857 (H)

Cheiromycina B. Sutton 1986 (H) 
Cheiropolyschema Matsush. 1980 (H)

Cheirospora Moug. \& Fr. 1825 (C)

Chiastospora Riess 1852 (C)

Chikaneea B. Sutton 1973 (H)

Chithramia Nag Raj 1988 (C)

Chlamydomyces Bainier 1907 (H)

Chlamydopsis Hol.-Jech. \& R.F. Castañeda 1986 (H)

Choanatiara DiCosmo 1984 (C)

Choreospora Constant. \& R. Sant. 1987 (H)

Chromosporium Corda 1829 (H)

Chrysachne Cif. 1938 (H)

Chrysalidopsis Steyaert 1961 (C)

Chryseidea Onofri 1981 (H)

Ciferria Gonz. Frag. 1925 (C)

Ciferriella Petr. 1930 (C)

Ciferrina Petr. 1932 (C)

Ciferriopeltis Bat. \& H. Maia 1965 (C)

Ciliochora Höhn. 1919 (C)

Ciliochorella Syd. 1935 (C)

Ciliophora Petr. 1929 (C)

Ciliophorella Petr. 1940 (C)

Ciliosporella Petr. 1927 (C)

Circinoconis Boedijn 1942 (H)

Circinotrichum Nees 1816 (H)

Cirrosporium S. Hughes 1980 (C)

Cissococcomyces Brain 1923

Civisubramaniania Vittal \& Dorai 1986 (H)

Cladochasiella Marvanová 1997 (H)

Cladoconidium Bandoni \& Tubaki 1985 (H)

Cladoniicola Diederich, van den Boom \& Aptroot 2001 (C)

Cladosporiella Deighton 1965 (H)

Clasteropycnis Bat. \& Cavalc. 1963 (C)

Clathroconium Samson \& H.C. Evans 1982 (H)

Clauzadeomyces Diederich 1994 (H)

Clavariana Nawawi 1976 (H)

Cleistocystis Sousa da Câmara 1931 (C)

Cleistophoma Petr. \& Syd. 1927 (C)

Clithramia Nag Raj 1988 (C)

Clohesyomyces K.D. Hyde 1993 (C)

Clypeochorella Petr. 1923 (C)

Clypeopatella Petr. 1942 (C)

Clypeophialophora Bat. \& Peres 1962 (C)

Clypeopycnis Petr. 1925 (C)

Clypeoseptoria F. Stevens \& P.A. Young 1925 (C)

Clypeostagonospora Punith. 1981 (C)
Mycosphere Doi 10.5943/mycosphere/3/2/5

Coccogloeum Petr. 1955 (C)

Codonmyces Calat. \& Etayo 1999 (H)

Colemaniella Agnihothr. 1974 (H)

Coleodictyospora Charles 1929 (H)

Coleophoma Höhn. 1907 (C)

Coleoseptoria Petr. 1940 (C)

Colispora Marvanová 1988 (H)

Collembolispora Marvanová \& Pascoal 2003 (H)

Colletoconis de Hoog \& Aa 1978 (C)

Colletosporium Link 1824 (H)

Collostroma Petr. 1947 (C)

Columnodomus Petr. 1941 (C)

Columnothyrium Bubák 1916 (C)

Comatospora Piroz. \& Shoemaker 1971 (C)

Comocephalum Syd. 1939 (C)

Condylospora Nawawi 1976 (H)

Coniambigua Etayo \& Diederich 1995 (C)

Conicomyces R.C. Sinclair, Eicker \& Morgan-Jones 1983 (H)

Conioscyphopsis Goh \& K.D. Hyde 1998

(H)

Coniothyrina Syd. 1912 (C)

Coniozyma Crous 2008 (C)

Conjunctospora Udagawa \& Uchiy. 1999

(H)

Conostoma Bat. \& J.L. Bezerra 1965 (C)

Consetiella Hol.-Jech. \& Mercado 1982

(H)

Coremiella Bubák \& K. Krieg. 1912 (H)

Cornucopiella Höhn. 1915 (C)

Cornutispora Piroz. 1973 (C)

Cornutostilbe Seifert 1990 (H)

Corynecercospora V.K. Pal, Akhtar, N.

Ahmad, Kamal \& D.K. Agarwal 2006

(H)

Corynesporella Munjal \& H.S. Gill 1961

(H)

Corynesporina Subram. 2009 (H)

Corynesporopsis P.M. Kirk 1981 (H)

Costanetoa Bat. \& J.L. Bezerra 1963 (C)

Craneomyces Morgan-Jones, R.C. Sinclair \& Eicker 1987 (H)

Creodiplodina Petr. 1957 (C)

Creonecte Petr. 1949 (C)

Creoseptoria Petr. 1937 (C)

Creothyriella Bat. \& C.A.A. Costa 1957

(C)

Cribropeltis Tehon 1933 (C)

Crinitospora B. Sutton \& Alcorn 1985 (C)

Crucellisporiopsis Nag Raj 1983 (C) 
Mycosphere Doi 10.5943/mycosphere/3/2/5

Crustodiplodina Punith. 1988 (C)

Cryptoceuthospora Petr. 1921 (C)

Cryptocoryneopsis B. Sutton 1980 (H)

Cryptocoryneum Fuckel 1870 (H)

Cryptophialoidea Kuthub. \& Nawawi 1987 (H)

Cryptosporium Kunze 1817 (C)

Cryptostroma P.H. Greg. \& S. Waller 1952 (H)

Cryptumbellata Udagawa \& Uchiy. 1999 (H)

Ctenosporium R. Kirschner 2006 (H)

Cubasina R.F. Castañeda 1986 (H)

Culicidospora R.H. Petersen 1960 (H)

Culicinomyces Couch, Romney \& B. Rao 1974 (H)

Curucispora Matsush. 1981 (H)

Curvulariopsis M.B. Ellis 1961 (H)

Cyanopatella Petr. 1949 (C)

Cyclomarsonina Petr. 1965 (C)

Cylindrium Bonord. 1851 (H)

Cylindrocarpostylus R. Kirschner \& Oberw. 1999 (H)

Cylindrodendrum Bonord. 1851 (H)

Cylindrogloeum Petr. 1941 (C)

Cylindromyces Manohar., D.K. Agarwal \& N.K. Rao 2004 (H)

Cylindrosympodium W.B. Kendr. \& R.F. Castañeda 1990 (H)

Cylindrothyrium Maire 1907 (C)

Cylindroxyphium Bat. \& Cif. 1963 (C)

Cymbothyrium Petr. 1947 (C)

Cyrenella Goch. 1981 (H)

Cystidiella Malan 1943 (Y)

Cystotrichiopsis B.A. Abbas, B. Sutton \& Ghaffar 2001 (C)

Cytodiscula Petr. 1931 (C)

Cytogloeum Petr. 1925 (C)

Cytonaema Höhn. 1914 (C)

Cytoplacosphaeria Petr. 1920 (C)

Cytopleastrum Abbas, B. Sutton, Ghaffar \& A. Abbas 2004 (C)

Cytosphaera Died. 1916 (C)

Cytosporella Sacc. 1884 (C)

Cytostagonospora Bubák 1916 (C)

Dactylifera Alcorn 1987 (H)

Dactylosporium Harz 1871 (H)

Dasysticta Speg. 1912 (C)

Davisiella Petr. 1924 (C)

Dearnessia Bubák 1916 (C)

Deccanodia Singhai 1974 (C)
Deichmannia Alstrup \& D. Hawksw. 1990 (H)

Delortia Pat. \& Gaillard $1888(\mathbf{H})$

Dendroclathra Voglmayr \& G. Delgado 2001 (H)

Dendrodomus Bubák 1915 (C)

Dendrographiella Agnihothr. 1972 (H)

Dendrographium Massee 1892 (H)

Dendroseptoria Alcalde 1948 (C)

Dendrospora Ingold 1943 (H)

Dendrosporium Plakidas \& Edgerton ex J.L. Crane 1972 (H)

Dendryphiosphaera Lunghini \& Rambelli 1978 (H)

Dennisographium Rifai 1977 (H)

Denticularia Deighton 1972 (H)

Dentocircinomyces R.F. Castañeda \& W.B. Kendr. 1990 (H)

Derexia Naras. 1970 (H)

Descalsia A. Roldán \& Honrubia 1989 (H)

Desertella Mouch. 1979 (H)

Desmidiospora Thaxt. 1891 (H)

Dexhowardia J.J. Taylor 1970 (H)

Diademospora B.E. Söderstr. \& Bååth 1979 (H)

Dialaceniopsis Bat. 1959 (C)

Diarimella B. Sutton 1980 (C)

Dichelostroma Bat. \& Peres 1963 (C)

Dicholobodigitus G.P. White \& Illman 1988 (H)

Dichotomophthora Mehrl. \& Fitzp. ex P.N. Rao 1966 (H)

Dichotomophthoropsis M.B. Ellis 1971 (H)

Dictyoarthrinium S. Hughes 1952 (H)

Dictyocatenulata Finley \& E.F. Morris 1967 (H)

Dictyodesmium S. Hughes 1951 (H)

Dictyophrynella Bat. \& Cavalc. 1964 (H)

Dictyopolyschema M.B. Ellis 1976 (H)

Dictyorostrella U. Braun 1999 (H)

Dictyospiropes M.B. Ellis 1976 (H)

Didymochaeta Sacc. \& Ellis 1898 (C)

Didymochaetina Bat. \& J.L. Bezerra 1965

(C)

Didymopsis Sacc. \& Marchal 1885 (H)

Didymosporina Höhn. 1916 (C)

Diedickea Syd. \& P. Syd. 1913 (C)

Digitatispora Doguet 1962 (H)

Digitodochium Tubaki \& Kubono 1989

(H) 
Digitomyces Mercado, M. Calduch \& Gené 2003 (H)

Digitopodium U. Braun, Heuchert \& K. Schub. 2005 (H)

Digitoramispora R.F. Castañeda \& W.B. Kendr. 1990 (H)

Dimastigosporium Faurel \& Schotter 1965 (C)

Diplocladiella G. Arnaud ex M.B. Ellis 1976 (H)

Diplodinis Clem. 1931 (C)

Diplodinula Tassi 1902 (C)

Diplolaeviopsis Giralt \& D. Hawksw. 1991 (C)

Diploplenodomus Died. 1912 (C)

Diplozythiella Died. 1916 (C)

Discomycetoidea Matsush. 1993 (H)

Discosiellina Subram. \& K.R.C. Reddy 1972 (C)

Discosporina Höhn. 1927 (C)

Discotheciella Syd. \& P. Syd 1917 (C)

Discozythia Petr. 1922 (C)

Dissitimurus E.g. Simmons, McGinnis \& Rinaldi 1987 (H)

Ditangifibula G.C. Adams 1996 (H)

Dokmaia I. Promputtha 2003 (H)

Domingoella Petr. \& Cif. 1932 (H)

Dothideodiplodia Murashk. 1927 (C)

Dothioropsis Riedl 1974 (C)

Drepanospora Berk. \& M.A. Curtis 1875 (H)

Drudeola Kuntze 1891 (C)

Drumopama Subram. 1957 (H)

Dryosphaera Jørg. Koch \& E.B.G. Jones 1989 (C)

Dualomyces Matsush. 1987 (H)

Dwayabeeja Subram. 1958 (H)

Dwayaloma Subram. 1957 (H)

Dwayalomella Brisson, Piroz. \& Pauzé 1975 (C)

Dwibahubeeja N. Srivast., A.K. Srivast. \& Kamal 1995 (H)

Dwibeeja Subram. 1995 (H)

Dwiroopella Subram. \& Muthumary 1986 (C)

Echinocatena R. Campb. \& B. Sutton 1977 (H)

Echinochondrium Samson \& Aa 1975 (H)

Echinoconidiophorum Pereira-Carv. \& Dianese 2009 (H)

Elachopeltella Bat. \& Cavalc. 1964 (C)

Elattopycnis Bat. \& Cavalc. 1964 (C)
Elegantimyces Goh, K.M. Tsui \& K.D. Hyde 1998 (H)

Eleutheromycella Höhn. 1908 (C)

Elletevera Deighton 1969 (H)

Ellisiella Bat. 1956 (H)

Elotespora R.F. Castañeda \& Heredia 2010 (H)

Enantioptera Descals 1983 (H)

Endobotrya Berk. \& M.A. Curtis 1874 (C)

Endobotryella Höhn. 1909 (C)

Endocalyx Berk. \& Broome 1876 (H)

Endoconospora Gjaerum 1971 (H)

Endocoryneum Petr. 1922 (C)

Endogenospora R.F. Castañeda, O. Morillo \& Minter 2010 (H)

Endophragmiopsis M.B. Ellis 1966 (H)

Endoplacodium Petr. 1949 (C)

Endoramularia Petr. 1923 (C)

Endosporoideus W.H. Ho, Yanna, K.D. Hyde \& Goh 2005 (H)

Endozythia Petr. 1959 (C)

Enerthidium Syd. 1939 (C)

Engelhardtiella A. Funk 1973 (H)

Enridescalsia R.F. Castañeda \& Guarro 1998 (H)

Enthallopycnidium F. Stevens 1925 (C)

Entoderma Hanula, Andreadis \& M. Blackw. 1991 (C)

Epaphroconidia Calat. \& V. Atienza 1995 (C)

Ephelidium C.W. Dodge \& E.D. Rudolph 1955 (C)

Epicladonia D. Hawksw. 1981 (C)

Epiclinium Fr. 1849 (C)

Epicoccospora Budathoki \& S.K. Singh 1995 (?C)

Epidermidophyton E. Lang 1879 (H)

Epidochiopsis P. Karst. 1892 (H)

Episporogoniella U. Braun 1994 (H)

Epistigme Syd. 1924 (C)

Eriocercospora Deighton 1969 (H)

Eriocercosporella Rak. Kumar, A.N. Rai \& Kamal ex U. Braun 1998 (H)

Ernakulamia Subram. 1996 (H)

Erysiphopsis Speg. 1910 (?C)

Erythrogloeum Petr. 1953 (C)

Esdipatilia Phadke 1981 (H)

Esteya J.Y. Liou, J.Y. Shih \& Tzean 1999

(H)

Eurotiopsis P. Karst. 1889 (?C)

Evanidomus Caball. 1941 (C)

Everhartia Sacc. \& Ellis 1882 (H) 
Everniicola D. Hawksw. 1982 (C)

Eversia J.L. Crane \& Schokn. 1977 (H)

Excipularia Sacc. 1884 (H)

Exochalara W. Gams \& Hol.-Jech. 1976 (H)

Exophoma Weedon 1926 (C)

Exosporella Höhn. 1912 (H)

Exosporium Link 1809 (H)

Falcocladium S.F. Silveira, Alfenas, Crous \& M.J. Wingf. 1994 (H)

Favostroma B. Sutton \& E.M. Davison 1983 (C)

Fecundostilbum Prameela \& Chowdhry 2009 (H)

Feltgeniomyces Dieder. 1990 (H)

Fenestroconidia Calat. \& Etayo 1999 (H)

Filosporella Nawawi 1976 (H)

Fissuricella Pore, D'Amatao \& Ajello 1977 (H)

Flabellocladia Nawawi 1985 (H)

Flabellospora Alas. 1968 (H)

Flosculomyces B. Sutton 1978 (H)

Frigidispora K.D. Hyde \& Goh 1999 (C)

Fujimyces Minter \& Caine 1980 (C)

Fuligomyces Morgan-Jones \& Kamal 1984 (H)

Fumagopsis Speg. 1910 (H)

Funicularius K.K. Baker \& Zaim 1979 (H)

Furcaspora Bonar 1965 (C)

Fusamen (Sacc.) P. Karst. 1890 (C)

Fusariella Sacc. 1884 (H)

Fusariopsis Horta 1919 (H)

Fuscophialis B. Sutton 1977 (H)

Fusicatena K. Matsush. \& Matsush. 1996 (H)

Fusicolla Bonord. 1851 (H)

Fusisporella Speg. 1910 (H)

Fusticeps J. Webster \& R.A. Davey 1980 (H)

Gamosporella Speg. 1888 (C)

Gampsonema Nag Raj 1975 (C)

Gangliophora Subram. 1992 (H)

Gangliostilbe Subram. \& Vittal 1976 (H)

Garnaudia Borowska 1977 (H)

Gaubaea Petr. 1942 (C)

Geastrumia Bat. 1960 (C)

Gelatinocrinis Matsush. 1995 (H)

Gelatinopycnis Dyko \& B. Sutton 1979

(C)

Geminoarcus K. Ando 1993 (H)

Gemmulina Descals \& Marvanová 1999
(H)

Geniopila Marvanová \& Descals 1985 (?C)

Gerulajacta Preuss 1855 (C)

Gilmaniella G.L. Barron 1964 (H)

Giulia Tassi 1904 (C)

Glaphyriopsis B. Sutton \& Pascoe 1987

(C)

Glenosporopsis O.M. Fonseca 1943 (H)

Glioannellodochium Matsush. 1989 (H)

Glioblastocladium Matsush. 1989 (H)

Globoconidiopsis G.F. Sepúlveda, Pereira-

Carv. \& Dianese 2009 (H)

Globoconidium G.F. Sepúlveda, PereiraCarv. \& Dianese 2009 (H)

Globuliroseum Sullia \& K.R. Khan 1984 (H)

Gloeodes Colby 1920 (C)

Gloeosporiella Cavara 1892 (?H)

Gloiosphaera Höhn. 1902 (H)

Glutinium Fr. 1849 (C)

Glycyphila Mont. 1851(H)

Goidanichiella G.L. Barron ex W. Gams 2009 (H)

Gonatobotryum Sacc. 1880 (H)

Gonatophragmiella Rak. Kumar, A.N. Rai \& Kamal 1994 (H)

Gonatorrhodum Corda 1839 (H)

Goniopila Marvanová \& Descals 1985 (H)

Goosiella Morgan-Jones, Kamal \& R.K. Verma 1986 (H)

Goosiomyces N.K. Rao \& Manohar. 1989 (H)

Gorgomyces M. Gönczöl \& Révay 1985 (H)

Grallomyces F. Stevens 1918 (H)

Granularia Sacc. \& Ellis ex Sacc. 1882

(H)

Graphiothecium Fuckel 1870 (H)

Groveolopsis Boedijn 1951 (C)

Guedea Rambelli \& Bartoli 1978 (H)

Gymnoxyphium Cif., Bat. \& I.J. Araujo 1963 (C)

Gyoerffyella Kol 1928 (H)

Gyrothrix (Corda) Corda 1842 (H)

Hadronema Syd. \& P. Syd. 1909 (H)

Hadrosporium Syd. 1938 (H)

Halysiomyces E.G. Simmons 1981 (H)

Halysium Corda 1837 (?H)

Hansfordia S. Hughes 1951 (H)

Hansfordiopeltis Bat. \& C.A.A. Costa 
1956 (C)

Hansfordiopeltopsis M.L. Farr 1986 (C)

Hapalosphaeria Syd. 1908 (C)

Haplariella Syd. \& P. Syd. 1908 (H)

Haplariopsis Oudem. 1903 (H)

Haplobasidion Erikss. 1889 (H)

Haplolepis Syd. 1925 (C)

Haplosporidium Speg. 1912 (C)

Haptocara Drechsler 1975 (H)

Harmoniella V.N. Boriss. 1981 (H)

Harpographium Sacc. 1880 (H)

Hawksworthiana U. Braun 1988 (H)

Heimiodora Nicot 1960 (H)

Helhonia B. Sutton 1980 (C)

Helicofilia Matsush. 1983 (H)

Helicogoosia Hol.-Jech. 1991 (H)

Helicomina L.S. Olive 1948 (H)

Helicominopsis Deighton 1960 (H)

Helicorhoidion S. Hughes 1958 (H)

Helicosingula P.S. van Wyk, Marasas, Baard \& Knox-Dav. 1985 (H)

Helicostilbe Höhn. 1902 (H)

Helicothyrium I. Hino \& Katum. 1961 (C)

Helicoübisia Lunghini \& Rambelli 1979 (H)

Heliscella Marvanová 1980 (H)

Heliscina Marvanová 1980 (H)

Helminthosporiomyces G.F. Sepúlveda,

Pereira-Carv. \& Dianese 2009 (H)

Hemibeltrania Piroz. 1963 (H)

Hemicorynesporella Subram. 1992 (H)

Hemidothis Syd. \& P. Syd. 1916 (C)

Hemisphaeropsis Petr. 1947 (C)

Hemisynnema Subram. 1995 (H)

Hendersoniella Tassi 1900 (C)

Hendersonina E.J. Butler 1913 (C)

Hendersoniopsis Höhn. 1918 (C)

Hendersonulina Petr. 1951 (C)

Henicospora P.M. Kirk \& B. Sutton 1980

(H)

Hermatomyces Speg. 1910 (H)

Herposira Syd. 1938 (H)

Herreromyces R.F. Castañeda \& W.B. Kendr. 1991 (H)

Heterocephalum Thaxt. 1903 (H)

Heteroconidium Sawada 1944 (H)

Heterosporiopsis Petr. 1950 (H)

Hexacladium D.L. Olivier 1983 (H)

Himantia Pers. 1801 (H)

Hinoa Hara \& I. Hino 1961

Hirudinaria Ces. 1856 (H)

Hoehneliella Bres. \& Sacc. 1902 (C)
Holubovaea Mercado 1983 (H)

Homalopeltis Bat. \& Valle 1961 (C)

Hormiactis Preuss 1851 (H)

Hormiscioideus M. Blackw. \& Kimbr. 1979 (H)

Hormocephalum Syd. 1939 (H)

Hormocladium Höhn. 1923 (H)

Hormococcus Robak 1956 (C)

Hormographis Guarro, Punsola \& Arx 1986 (H)

Hughesinia J.C. Lindq. \& Gamundí 1970 (H)

Humicolopsis Verona 1977 (H)

Hyalocamposporium Révay \& J. Gönczöl 2007 (H)

Hyalocladium Mustafa 1977 (H)

Hyalocylindrophora J.L. Crane \& Dumont 1978 (H)

Hyalodictyum Woron. 1916 (C)

Hyalohelicomina T. Yokoy. 1974 (H)

Hyalopleiochaeta R.F. Castañeda, Guarro \& Cano 1996 (H)

Hyalosynnema Matsush. 1975 (H)

Hyalothyridium Tassi 1900 (C)

Hyalotiastrum Abbas, B. Sutton, Ghaffar \& Abbas 2003 (C)

Hyalotiella Papendorf 1967 (C)

Hyalotrochophora Finley \& E.F. Morris 1967 (H)

Hydrometrospora J. Gönczöl \& Révay 1985 (H)

Hymenella Fr. 1822 (H)

Hymeniopeltis Bat. 1959 (C)

Hymenobactron (Sacc.) Höhn. 1916 (H)

Hymenopsis Sacc. 1886 (C)

Hyphochlaena Cif. 1962 (H)

Hyphodiscosia Lodha \& K.R.C. Reddy 1974 (H)

Hyphodiscosioides Matsush. 1993 (H)

Hyphopolynema Nag Raj 1977 (H)

Hyphostereum Pat. 1892 (C)

Hyphothyrium B. Sutton \& Pascoe 1989 (H)

Hypocline Syd. 1939 (C)

Hypogloeum Petr. 1923 (C)

Hysteridium P. Karst. 1905 (C)

Hysteropycnis Hilitzer 1929 (C)

Ialomitzia Gruia 1964 (H)

Icerymyces Brain 1923 (C)

Idiocercus B. Sutton 1967 (C)

Impudentia Vujanović 2003 (H)

Inesiosporium R.F. Castañeda \& W. Gams 
1997 (H)

Infundibulomyces Plaingam, Somrith. \& E.B.G. Jones 2003 (C)

Inifatiella R.F. Castañeda 1985 (H)

Intercalarispora J.L. Crane \& Schokn. 1983 (H)

Intralichen D. Hawksw. \& M.S. Cole 2002 (H)

Ionophragmium Peres 1961 (C)

Irpicomyces Deighton 1969 (H)

Isariella Henn. 1908 (H)

Ischnostroma Syd. \& P. Syd. 1914 (H)

Isthmolongispora Matsush. 1971 (H)

Isthmophragmospora Kuthub. \& Nawawi 1992 (H)

Isthmotricladia Matsush. 1971(H)

Ityorhoptrum P.M. Kirk 1986 (H)

Iyengarina Subram. 1958 (H)

Jahniella Petr. 1921 (C)

Janetia M.B. Ellis 1976 (H)

Javonarxia Subram. 1995 (H)

Jerainum Nawawi \& Kuthub. 1992 (H)

Jubispora B. Sutton \& H.J. Swart 1986 (C)

Junctospora Minter \& Hol.-Jech. 1981 (H)

Junewangia W.A. Baker \& Morgan-Jones 2002 (H)

Kalamarospora G. Delgado 2011 ('2010) (H)

Kalchbrenneriella Diederich \& M.S. Christ. 2002 (H)

Kaleidosporium Van Warmelo \& B. Sutton 1981 (C)

Kamatella Anahosur 1969 (C)

Kamatia V.G. Rao \& Subhedar 1976 (H)

Kameshwaromyces Kamal, R.K. Verma \& Morgan-Jones 1986 (H)

Karsteniomyces D. Hawksw. 1980 (C)

Kendrickiella K. Jacobs \& M.J. Wingf. 2001 (H)

Ketubakia Kamat, Varghese \& V.G. Rao 1987 (H)

Kiliophora Kuthub. \& Nawawi 1993 (H)

Kionocephala P.M. Kirk 1986 (H)

Kmetia Bres. \& Sacc. 1902 (H)

Kmetiopsis Bat. \& Peres 1960 (H)

Knemiothyrium Bat. \& J.L. Bezerra 1960 (C)

Knufia L.J. Hutchison \& Unter. 1996 (H)

Kodonospora K. Ando 1993 (H)

Kolletes Kohlm. \& Volkm.-Kohlm. 2005

(H)
Kontospora A. Roldán, Honrubia \& Marvanová 1990 (H)

Koorchalomella Chona, Munjal \& J.N. Kapoor 1958 (H)

Korunomyces Hodges \& F.A. Ferreira 1981 (H)

Kostermansinda Rifai 1968 (H)

Kostermansindiopsis R.F. Castañeda 1986 (H)

Kramabeeja G.V. Rao \& K.A. Reddy 1981 (H)

Kramasamuha Subram. \& Vittal 1973 (H)

Kreiseliella Braun 1991 (C)

Krishnamyces Hosag. 2003 (C)

Kumanasamuha P.Rag. Rao \& D. Rao 1964 (H)

Kyphophora B. Sutton 1991 (C)

Lacellina Sacc. 1913 (H)

Lacellinopsis Subram. 1953 (H)

Lachnodochium Marchal 1895 (H)

Laciniocladium Petri 1917 (H)

Laeviomyces D. Hawksw. 1981 (C)

Lagenomyces Cavalc. \& A.A. Silva 1972 (C)

Lambdasporium Matsush. 1971 (H)

Lambinonia Sérus. \& Diederich 2005 (H)

Lamproconium (Grove) Grove 1937 (C)

Lappodochium Matsush. 1975 (H)

Lasiodiplodiella Zambett. 1955 (C)

Lasiophoma Speg. 1918 (C)

Lasiothyrium Syd. \& P. Syd. 1913 (C)

Lasmenia Speg. 1886 (C)

Lasmeniella Petr. \& Syd. 1927 (C)

Latericonis G.V. Rao, K.A. Reddy \& de Hoog 1984 (H)

Lateriramulosa Matsush. 1971 (H)

Laterispora Uecker, W.A. Ayers \& P.B. Adams 1982 (H)

Lauriomyces R.F. Castañeda 1990 (H)

Lawalreea Dieder. 1990 (C)

Leandria Rangel 1915 (H)

Lecaniocola Brain 1923 (?C)

Lecanostictopsis B. Sutton \& Crous 1997 (H)

Lecophagus M.W. Dick 1990 (H)

Lecythispora Chowdhry 1985 (?C)

Lecythophora Nannf. 1934, anamorphic Igneocumulus A.W. Ramaley 2003 (H)

Leeina Petr. 1923 (C)

Leightoniomyces D. Hawksw. \& B. Sutton 1977 (H)

Lembuncula Cif. 1954 (C) 
Mycosphere Doi 10.5943/mycosphere/3/2/5

Lemkea Morgan-Jones \& R.C. Sinclair 1983 (H)

Lepisticola W. Gams 2009 (H)

Leprieurinella Bat. \& H. Maia 1961 (C)

Leptochlamys Died. 1921(C)

Leptodermella Höhn. 1915 (C)

Leptodiscella Papendorf 1969 (H)

Leptomelanconium Petr. 1923 (C)

Leptophyllosticta I.E. Brezhnev 1939 (C)

Leptostromella (Sacc.) Sacc. 1884 (C)

Leptothyrella Sacc. 1885 (C)

Leptothyrina Höhn. 1915 (C)

Leptothyrium Kunze 1823 (C)

Leucodochium Syd. \& P. Syd. 1917 (H)

Leucopenicillifer G.R.W. Arnold 1971 (H)

Leuliisinea Matsush. 1985 (H)

Libertiella Speg. \& Roum. 1880 (H)

Lichenobactridium Diederich \& Etayo 1995 (H)

Lichenoconium Petr. \& Syd. 1927 (C)

Lichenodiplis Dyko \& D. Hawksw. 1979 (C)

Lichenodiplisiella S.Y. Kondr. \& Kudratov 2002 (C)

Lichenohendersonia Calat. \& Etayo 2001 (C)

Lichenopuccinia D. Hawksw. \& Hafellner 1984 (H)

Lichenostella Calat. \& Etayo 1999 (H)

Lichenosticta Zopf 1898 (C)

Linochorella Syd. \& P. Syd. 1912 (C)

Listeromyces Penz. \& Sacc. 1901 (H)

Lobatopedis P.M. Kirk 1979 (H)

Loliomyces Maire 1937 (H)

Lomaantha Subram. 1954 (H)*

Lomachashaka Subram. 1956 (H)

Lunulospora Ingold 1942 (H)

Luxuriomyces R.F. Castañeda 1988 (H)

Luzfridiella R.F. Castañeda \& W.B. Kendr. 1991 (H)

Lylea Morgan-Jones 1975 (H)

Lysotheca Cif. 1962 (C)

Mackenziella Yanna \& K.D. Hyde 2009 (H)

Macrodiplodia Sacc. 1884 (C)

Macrodiplodiopsis Petr. 1922 (C)

Macrotrichum Grev. 1825 (H)

Mahabalella B. Sutton \& S.D. Patil 1966 (H)

Manginella Bat. \& H. Maia 1961 (C)

Manoharachariella Bagyan., N.K. Rao \& Kunwar 2009 (H)
Mapletonia B. Sutton 1991 (C)

Margarinomyces Laxa 1930 (H)

Martinellisia V.G. Rao \& Varghese 1977 (H)

Massalongina Bubák 1916 (C)

Massariothea Syd. 1939 (C)

Mastigosporium Riess 1852 (H)

Matsushimaea Subram. 1978 (H)

Matsushimiella R.F. Castañeda \& Heredia 2001 (H)

Matsushimomyces V.G. Rao \& Varghese 1979 (H)

Medusamyces G.L. Barron \& Szijarto 1990 (H)

Megacapitula J.L. Chen \& Tzean 1993 (H)

Megalodochium Deighton 1960 (H)

Melanocephala S. Hughes 1979 (H)

Melanodiscus Höhn. 1918 (H)

Melanophoma Papendorf \& J.W. du Toit 1967 (C)

Melanosella Örösi-Pál 1936 (H)

Melophia Sacc. 1884 (C)

Menidochium R.F. Castañeda \& W.B. Kendr. 1990 (H)

Menoidea L. Mangin \& Har. 1907 (H)

Mercadomyces J. Mena 1988 (H)

Metadiplodia Syd. 1937 (C)

Metarhiziopsis D.W. Li, R.S. Cowles \& Vossbrinck 2008 (H)

Metazythia Petr. 1950 (C)

Metazythiopsis M. Morelet 1988 (C)

Microblastosporon Cif. 1930 (H)

Microclava F. Stevens 1917 (H)

Microdiscula Höhn. 1915 (C)

Microdothiorella C.A.A. Costa \& Sousa da Câmara 1955 (C)

Microhendersonula Dias \& Sousa da Câmara 1952 (C)

Microperella Höhn. 1909 (C)

Microtyle Speg. 1919 (C)

Microxyphiella Speg. 1918 (C)

Microxyphiopsis Bat. 1963 (C)

Milospium D. Hawksw. 1975 (H)

Mindoa Petr. 1949 (C)

Miniancora Marvanová \& Bärl. 1989 (H)

Minimelanolocus R.F. Castañeda \& Heredia $2001(\mathbf{H})$

Minimidochium B. Sutton 1969 (H)

Minutoexcipula V. Atienza \& D. Hawksw. 1994 (H)

Minutophoma D. Hawksw. 1981 (C)

Mirimyces Nag Raj 1993 (C) 
Mixtoconidium Etayo 1995 (C)

Mohgaonidium Singhai 1974 (?C)

Monochaetiella E. Castell. 1943 (C)

Monochaetopsis Pat. 1931(H)

Monodia Breton \& Faurel 1970 (C)

Monodidymaria U. Braun 1994 (H)

Monodisma Alcorn 1975 (H)

Monosporiella Speg. 1918 (H)

Monosporium Bonord. 1851 (H)

Monotretomyces Morgan-Jones, R.C. Sinclair \& Eicker 1987 (H)

Monotrichum Gäum. 1922 (H)

Moralesia Urries 1956 (C)

Morosporium Renault \& Roche 1898 (?C)

Morrisographium M. Morelet 1968 (H)

Mucosetospora M. Morelet 1972 (C)

Muiogone Thaxt. 1914 (H)

Muirella R. Sprague 1959 (H)

Murogenella Goos \& E.F. Morris 1965 (H)

Mycelephas R.F. Castañeda 2009 (H)

Mycocentrodochium K. Matsush. \& Matsush. 1996 (H)

Mycochlamys S. Marchand \& Cabral 1976 (H)

Mycoënterolobium Goos 1970 (H)

Mycofalcella Marvanová, Om-Kalth. \& J. Webster 1993 (H)

Mycomyces Wyss-Chod. 1928 (H)

Mycopara Bat. \& J.L. Bezerra 1960 (C)

Mycospraguea U. Braun \& Rogerson 1993 (C)

Mycosticta Höhn. 1918 (C)

Mycosisymbrium Carris 1994 (H)

Mycosylva M.C. Tulloch 1973 (H)

Mycotribulus Nag Raj \& W.B. Kendr. 1970 (C)

Mycousteria M.L. Farr 1986 (C)

Myiocoprula Petr. 1955 (C)

Myriellina Höhn. 1915 (C)

Myrmecomyces Jouvenaz \& Kimbr. 1991 (H)

Myrotheciastrum Abbas \& B. Sutton 1988 (H)

Mystrosporiella Munjal \& Kulshr. 1969 (H)

Myxocephala G. Weber, Spaaij \& Oberw. 1989 (H)

Myxoparaphysella Caball. 1941 (C)

Myxosporella Sacc. 1881 (C)

Myxosporidiella Negru 1960 (C)

Myxostomellina Syd. 1931 (C)
Mycosphere Doi 10.5943/mycosphere/3/2/5

Myxothyriopsis Bat. \& A.F. Vital 1956 (C)

Myxothyrium Bubák \& Kabát 1915 (C)

Naemaspora Pers. 1796 (?C)

Naemosphaera P. Karst. 1888 (C)

Naemosphaerella Höhn. 1923 (C)

Nagrajia R.F. Castañeda \& W.B. Kendr. 1991 (C)

Nagrajomyces Melnik 1984 (C)

Nakatopsis Whitton, McKenzie \& K.D. Hyde 2001 (H)

Nanoschema B. Sutton 1980 (C)

Naothyrsium Bat. 1960 (C)

Navaneethospora V.G. Rao 1994 (H)

Necraphidium Cif. 1951 (H)

Nematogonum Desm. 1834 (H)

Nematographium Goid. 1935 (H)

Nemozythiella Höhn. 1925 (C)

Neoalpakesa Punith. 1981 (C)

Neoarbuscula B. Sutton 1983 (H)

Neobarclaya Sacc. 1899 (C)

Neodiplodina Petr. 1954 (C)

Neofuckelia Zeller \& Goodd. 1935 (C)

Neohendersonia Petr. 1921 (C)

Neoheteroceras Nag Raj 1993 (C)

Neojohnstonia B. Sutton 1983 (H)

Neoligniella Naumov 1951 (C)

Neomarssoniella U. Braun 1991 (C)

Neomelanconium Petr. 1940 (C)

Neoovularia U. Braun 1992 (H)

Neopeltis Syd. 1937 (C)

Neopericonia Kamal, A.N. Rai \& MorganJones 1983 (H)

Neophoma Petr. \& Syd. 1927 (C)

Neoplaconema B. Sutton 1977 (C)

Neoramularia U. Braun 1991 (H)

Neospegazzinia Petr. \& Syd. 1936 (C)

Neosporidesmium Mercado \& J. Mena 1988 (H)

Neottiospora Desm. 1843 (C)

Neta Shearer \& J.L. Crane 1971 (H)

Nidulispora Nawawi \& Kuthub. 1990 (H)

Nigromacula Etayo 2002 (H)

Nigropuncta D. Hawksw. 1981 (C)

Nosophloea Fr. 1849 (?C)

Nothospora Peyronel 1913 (H)

Novozymia W.P. Wu 2005 (H)

Nummospora E. Müll. \& Shoemaker 1964

(C)

Nusia Subram. 1995 (H)

Nyctalospora E.F. Morris 1972 (H)

Obeliospora Nawawi \& Kuthub. 1990 (H)

Obstipipilus B. Sutton 1968 (C) 
Octopodotus Kohlm. \& Volkm.-Kohlm. 2003 (C)

Odontodictyospora Mercado 1984 (H)

Oedothea Syd. 1930 (H)

Ojibwaya B. Sutton 1973 (H)

Olpitrichum G.F. Atk. 1894 (H)

Omega B. Sutton \& Minter 1988 (C)

Oncocladium Wallr. 1833 anamorphic

Gymnoascus Baran. 1872 (H)

Oncopodiella G. Arnaud ex Rifai 1965 (H)

Oncopodium Sacc. 1904 (H)

Oncospora Kalchbr. 1880 (C)

Oncosporella P. Karst. 1887 (C)

Oncostroma Bat. \& Marasas 1966 (C)

Onychophora W. Gams, P.J. Fisher \& J. Webster 1984 (H)

Oothyrium Syd. 1939 (C)

Ophiosira Petr. 1955 (C)

Orbimyces Linder 1944 (H)

Ostracodermidium Mukerji 1973 (H)

Ovadendron Sigler \& J.W. Carmich. 1976 (H)

Ozonium Link 1809 (H)

Paathramaya Subram. 1956 (H)

Pachycladina Marvanová 1987 (H)

Pactilia Fr. 1837 (H)

Palawaniopsis Bat., Cif. \& Nascim. 1959

Pantospora Cif. 1938 (H)

Papilionospora V.G. Rao \& B. Sutton 1976 (H)

Pappimyces B. Sutton \& Hodges 1975 (H)

Paraaoria R.K. Verma \& Kamal 1987

Paraarthrocladium Matsush. 1993 (H)

Parabeltrania Rambelli 2008 (H)

Paraceratocladium R.F. Castañeda 1987 (H)

Parachionomyces Thaung 1979 (H)

Paracostantinella Subram. \& Sudha 1989 (H)

Paracryptophiale Kuthub. \& Nawawi 1994 (H)

Paracytospora Petr. 1925 (C)

Paradendryphiopsis M.B. Ellis 1976 (H)

Paradictyoarthrinium Matsush. 1996 (H)

Paradidymobotryum C.J.K. Wang \& B. Sutton 1984 (H)

Paradiplodia Speg. ex Trotter 1931 (C)

Paradischloridium Bhat \& B. Sutton 1985 (H)

Paradiscula Petr. 1941(C)

Paraëpicoccum Matsush. 1993 (H)

Parafulvia Kamal, A.N. Rai \& Morgan-
Jones 1983 (H)

Parahaplotrichum W.A. Baker \& Partr. 2001 (H)

Paraharknessia Matsush. 2003 (H)

Parahyalotiopsis Nag Raj 1976 (C)

Paramassariothea Subram. \& Muthumary 1979 (C)

Paramenisporopsis Matsush. 2003 (H)

Parapericonia M.B. Ellis 1976 (H)

Parapericoniella U. Braun, Heuchert \& K. Schub. 2005 (H)

Paraphaeoisaria de Hoog \& MorganJones 1978 (H)

Parapithomyces Thaung 1976 (H)

Parapleurotheciopsis P.M. Kirk 1982 (H)

Parapyricularia M.B. Ellis 1972 (H)

Pararobillarda Matsush. 1996 (C)

Parasphaeropsis Petr. 1953 (C)

Paraspora Grove 1884 (H)

Parastigmatellina Bat. \& C.A.A. Costa 1959 (C)

Parasympodiella Ponnappa 1975 (H)

Paratetraploa M.K.M. Wong \& K.D. Hyde 2002 (H)

Paratomenticola M.B. Ellis 1976 (H)

Paratrichoconis Deighton \& Piroz. 1972 (H)

Paraülocladium R.F. Castañeda 1986 (H)

Paspalomyces Linder 1933 (H)

Patouillardiella Speg. 1889 (H)

Patriciomyces D. Hawksw. 2001(H)

Peethasthabeeja P.Rag. Rao 1981 (H)

Pellionella (Sacc.) Sacc. 1902 (C)

Peltasterinostroma Punith. 1975 (C)

Peltasteropsis Bat. \& H. Maia 1959 (C)

Peltistroma Henn. 1904 (C)

Peltistromella Höhn. 1907 (C)

Peltosoma Syd. 1925 (C)

Peltostromellina Bat. \& A.F. Vital 1959

(C)

Peltostromopsis Bat. \& A.F. Vital 1959

(C)

Penzigomyces Subram. 1992 (H)

Perelegamyces R.F. Castañeda \& W.B.

Kendr. 1990 (H)

Perizomella Syd. 1927 (C)

Pestalozziella Sacc. \& Ellis ex Sacc. 1882

(C)

Petrakia Syd. \& P. Syd. 1913 (H)

Petrakiopsis Subram. \& K.R.C. Reddy 1968 (H)

Phacostroma Petr. 1955 (C) 
Phacostromella Petr. 1955 (C)

Phaeoblastophora Partr. \& Morgan-Jones 2002 (H)

Phaeobotrys M. Calduch, Gené \& Guarro 2002 (H)

Phaeocandelabrum R.F. Castañeda, Heredia, Saikawa 2009 (H)

Phaeocytostroma Petr. 1921 (C)

Phaeodactylella Udaiyan 1992 (H)

Phaeodactylium Agnihothr. 1968 (H)

Phaeodiscula Cub. 1891 (C)

Phaeodomus Höhn. 1909 (C)

Phaeohiratsukaea Udagawa \& Iwatsu 1990 (H)

Phaeohymenula Petr. 1954 (H)

Phaeoidiomyces Dorn.-Silva \& Dianese 2004 (H)

Phaeolabrella Speg. 1912 (C)

Phaeomonilia R.F. Castañeda, Heredia \& R.M. Arias 2007 (H)

Phaeomonostichella Keissl. ex Petr. 1941 (C)

Phaeophloeosporella Crous \& B. Sutton 1997 (C)

Phaeophomopsis Höhn. 1917 (C)

Phaeosphaera Bat. \& Cif. 1963 (C)

Phaeosporobolus D. Hawksw. \& Hafellner 1986 (H)

Phaeothyrium Petr. 1947 (C)

Phaeostilbella Höhn. 1919 (H)

Phaeotrichoconis Subram. 1956 (H)

Phalangispora Nawawi \& J. Webster 1982 (H)

Phialoarthrobotryum Matsush. 1975 (H)

Phialogangliospora Udaiyan \& V.S. Hosag. 1992 (H)

Phialomyces P.C. Misra \& P.H.B. Talbot 1964 (H)

Phialophaeoisaria Matsush. 1995 (H)

Phialoselanospora Udaiyan 1992 (H)

Phialosporostilbe Mercado \& J. Mena 1985 (H)

Phialostele Deighton 1969 (H)

Phialotubus R.Y. Roy \& Leelav. 1966 (H)

Phloeoconis Fr. 1849 (H)

Phloeosporina Höhn. 1924 (C)

Phlyctaeniella Petr. 1922 (C)

Phomachora Petr. \& Syd. 1925 (C)

Phomachorella Petr. 1947 (C)

Phomyces Clem. 1931 (C)

Phragmocephala E.W. Mason \& S. Hughes 1951 (H)
Mycosphere Doi 10.5943/mycosphere/3/2/5

Phragmoconidium G.F. Sepúlveda, Pereira-Carv. \& Dianese 2009 (H)

Phragmogloeum Petr. 1954 (C)

Phragmopeltis Henn. 1904 (C)

Phragmospathula Subram. \& N.G. Nair 1966 (H)

Phragmospathulella J. Mena \& Mercado 1986 (H)

Phragmotrichum Kunze 1823 (C)

Phylloedium Fr. 1825 (H)

Phyllohendersonia Tassi 1902 (C)

Physalidiella Rulamort 1990 (H)

Physalidiopsis R.F. Castañeda \& W.B. Kendr. 1990 (H)

Pilulina G. Arnaud 1954 (H)

Pinatubo J.B. Manandhar \& Mew 1996 (H)

Piperivora Siboe, P.M. Kirk \& P.F. Cannon 1999 (H)

Piricauda Bubák 1914 (H)

Piricaudilium Hol.-Jech. 1988 (H)

Piricaudiopsis J. Mena \& Mercado 1987 (H)

Pirispora Faurel \& Schotter 1966 (C)

Pirostomella Sacc. 1914 (H)

Pirozynskiella S. Hughes 2007 (H)

Pittostroma Kowalski \& T.N. Sieber 1992 (C)

Placella Syd. 1938 (C)

Placodiplodia Bubák 1916 (C)

Placonema (Sacc.) Petr. 1921 (C)

Placonemina Petr. 1921 (C)

Plagiostigmella Petr. 1949 (C)

Plectonaemella Höhn. 1915 (C)

Plectopeltis Syd. 1927 (C)

Plectophomella Moesz 1922 (H)

Plectophomopsis Petr. 1922 (C)

Plectosira Petr. 1929 (C)

Plectronidiopsis Nag Raj 1979 (C)

Plectronidium Nag Raj 1977 (C)

Pleiochaeta (Sacc.) S. Hughes 1951 (H)

Plenocatenulis Bat. \& Cif. 1959 (C)

Plenophysa Syd. \& P. Syd. 1920 (C)

Plenotrichopsis Bat. 1961 (C)

Plenotrichum Syd. 1927 (C)

Plenozythia Syd. \& P. Syd. 1916 (C)

Pleocouturea G. Arnaud 1911 (C)

Plesiospora Drechsler 1971 (H)

Pleurodiscula Höhn. 1926 (C)

Pleurodiscus Petr. 1931 (C)

Pleurodomus Petr. 1934 (C)

Pleuropedium Marvanová \& S.H. Iqbal 
1973 (H)

Pleurophragmium Costantin 1888 (H)

Pleuroplaconema Petr. 1923 (C)

Pleuroplacosphaeria Syd. 1928 (C)

Pleurotheciopsis B. Sutton 1973 (H)

Pleurothyriella Petr. \& Syd. 1925 (C)

Pleurothyrium Bubák 1916 (C)

Pleurovularia R. Kirschner \& U. Braun 2002 (H)

Pocillopycnis Dyko \& B. Sutton 1979 (C)

Podosporiella Ellis \& Everh. 1894 (H)

Podosporium Schwein. 1832 (H)

Poikilosperma Bat. \& J.L. Bezerra 1961 (C)

Polyancora Voglmayr \& C. Yule 2006 (H)

Polybulbophiale Goh \& K.D. Hyde 1998 (H)

Polycladium Ingold 1959 (H)

Polydesmus Mont. 1845 (H)

Polyetron Bat. \& Peres 1963 (C)

Polylobatispora Matsush. 1996 (H)

Polyscytalum Riess 1853 (H)

Polystomellomyces Bat. 1959 (C)

Polystratorictus Matsush. 1993 (H)

Polythrinciella Bat. \& H. Maia 1960 (H)

Polythrinciopsis J. Walker 1966 (H)

Polytretophora Mercado 1983 (H)

Porobeltraniella Gusmão 2004 (H)

Porocladium Descals 1976 (H)

Poropeltis Henn. 1904 (C)

Porophilomyces U. Braun 2000 (H)

Porosubramaniania Hol.-Jech. 1985 (H)

Porrectotheca Matsush. 1996 (C)

Powellia Bat. \& Peres 1964 (C)

Prathigada Subram. 1956 (H)

Prismaria Preuss 1851 (H)

Proboscispora Punith. 1984 (C)

Prophytroma Sorokīn 1877 (H)

Prosthemiella Sacc. 1881 (H)

Protostegia Cooke 1880 (C)

Protostegiomyces Bat. \& A.F. Vital 1955 (C)

Protostroma Bat. 1957 (C)

Psammina Sacc. \& M. Rousseau ex E. Bommer \& M. Rousseau 1891 (C)

Pseudoacrodictys W.A. Baker \& MorganJones 2003 (H)

Pseudoanguillospora S.H. Iqbal 1974 (H)

Pseudoaristastoma Suj. Singh 1979 (C)

Pseudoasperisporium U. Braun 2000 (H)

Pseudobasidiospora Dyko \& B. Sutton 1978 (C)
Mycosphere Doi 10.5943/mycosphere/3/2/5

Pseudobeltrania Henn. 1902 (H)

Pseudocanalisporium R.F. Castañeda \& W.B. Kendr. 1991 (H)

Pseudocenangium P. Karst. 1886 (C)

Pseudochuppia Kamal, A.N. Rai \& Morgan-Jones 1984 (H)

Pseudoclathrosphaerina Voglmayr 1997 (H)

Pseudoconium Petr. 1969 (C)

Pseudocytoplacosphaeria Punith. \& Spooner 2002 (?C)

Pseudocytospora Petr. 1923 (C)

Pseudodichomera Höhn. 1918 (C)

Pseudodidymaria U. Braun 1993 (H)

Pseudodiplodia (P. Karst.) Sacc. 1884 (C)

Pseudodiscula Laubert 1911 (C)

Pseudoepicoccum M.B. Ellis 1971 (H)

Pseudofuscophialis Sivan. \& H.S. Chang 1995 (H)

Pseudogaster Höhn. 1907 (H)

Pseudogliophragma Phadke \& V.G. Rao 1980 (H)

Pseudographiella E.F. Morris 1966 (H)

Pseudohendersonia Crous \& M.E. Palm 1999 (C)

Pseudolachnea Ranoj. 1910 (C)

Pseudoneottiospora Faurel \& Schotter 1965 (C)

Pseudopapulaspora N.D. Sharma 1977 (H)

Pseudopatellina Höhn. 1908 (C)

Pseudopeltistroma Katum. 1975 (C)

Pseudopetrakia M.B. Ellis 1971 (H)

Pseudophloeosporella U. Braun 1993 (C)

Pseudophragmotrichum W.P. Wu, B.

Sutton \& Gange 1998 (C)

Pseudopolystigmina Murashk. 1928 (C)

Pseudoramularia Matsush. 1983 (H)

Pseudorhizopogon Kobayasi 1983 (C)

Pseudoschizothyra Punith. 1980 (C)

Pseudoseptoria Speg. 1910 (C)

Pseudosigmoidea K. Ando \& N. Nakam. 2000 (H)

Pseudostegia Bubák 1906 (C)

Pseudothyrium Höhn. 1927 (C)

Pseudotorula Subram. 1958 (H)

Pseudotracylla B. Sutton \& Hodges 1976

(C)

Pseudotrichoconis W.A. Baker \& MorganJones 2001 (H)

Pseudozythia Höhn. 1902 (C) 
Pterulopsis Wakef. \& Hansf. 1943 (H)

Pterygosporopsis P.M. Kirk 1983 (H)

Pucciniospora Speg. 1886 (C)

Pulchromyces Hennebert 1973 (H)

Pullospora Faurel \& Schotter 1965 (C)

Pulvinella A.W. Ramaley 2001 (H)

Punctillina Toro 1934 (C)

Pycnidioarxiella Punith. \& N.D. Sharma 1980 (C)

Pycnidiopeltis Bat. \& C.A.A. Costa 1959 (C)

Pycnis Bref. 1881 (C)

Pycnodactylus Bat., A.A. Silva \& Cavalc. 1967 (C)

Pycnodallia Kohlm. \& Volkm.-Kohlm. 2001 (?C)

Pycnoharknessia Matsush. 1996 (C)

Pycnomma Syd. 1924 (C)

Pycnomoreletia Rulamort 1990 (C)

Pycnopleiospora C.Z. Wei, Y. Harada \& Katum. 1997 (H)

Pycnoseynesia Kuntze 1898 (C)

Pycnothera N.D. Sharma \& G.P. Agarwal 1974 (C)

Pycnothyriella Bat. 1952 (C)

Pycnothyrium Died. 1913 (C)

Pyramidospora Sv. Nilsson 1962 (H)

Pyrenyllium Clem. 1909 (?C)

Pyrgostroma Petr. 1951 (C)

Pyriculariopsis M.B. Ellis 1971 (H)

Pyripnomyces Cavalc. 1972 (C)

Quadracaea Lunghini, Pinzari \& Zucconi 1996 (H)

Quadricladium Nawawi \& Kuthub. 1989 (H)

Quasidiscus B. Sutton 1991 (C)

Queenslandia Bat. \& H. Maia 1959 (C)

Quezelia Faurel \& Schotter 1965 (C)

Radiatispora Matsush. 1995 (H)

Radiciseta Sawada \& Katsuki 1959 (H)

Radulidium Arzanlou, W. Gams \& Crous 2007 (H)

Raizadenia S.L. Srivast. 1981 (H)

Ramakrishnanella Kamat \& Ullasa ex Ullasa 1970 (C)

Ramicephala Voglmayr \& G. Delgado 2003 (H)

Ramoconidiifera B. Sutton, Carmarán \& A.I. Romero 1996 (H)

Ranojevicia Bubák 1910 (H)

Redbia Deighton \& Piroz. 1972 (H)

Refractohilum D. Hawksw. 1977 (H)
Mycosphere Doi 10.5943/mycosphere/3/2/5

Reichlingia Diederich \& Scheid. 1996 (H)

Remersonia Samson \& Seifert 1997 (H)

Repetoblastiella R.F. Castañeda, Minter \&

M. Stadler 2010 (H)

Retiarius D.L. Olivier 1978 (H)

Rhabdoclema Syd. 1939 (C)

Rhabdostromella Höhn. 1915 (C)

Rhacodiella Peyronel 1919 (H)

Rhexoacrodictys W.A. Baker \& MorganJones 2002 (H)

Rhexoampullifera P.M. Kirk 1982 (H)

Rhexodenticula W.A. Baker \& MorganJones 2001 (H)

Rhexoprolifer Matsush. 1996 (H)

Rhinocladium Sacc. \& Marchal 1885 (H)

Rhinotrichella G. Arnaud ex de Hoog 1977 (H)

Rhipidocephalum Trail 1888 (H)

Rhizohypha Chodat \& Sigr. 1911 (?C)

Rhizosphaerina B. Sutton 1986 (?C)

Rhodesiopsis B. Sutton \& R. Campb. 1979

(C)

Rhodothallus Bat. \& Cif. 1959 (H)

Rhombostilbella Zimm. 1902 (H)

Rhynchodiplodia Briosi \& Farneti 1906 (C)

Rhynchomyces Willk. 1866 (H)

Rhynchoseptoria Unamuno 1940 (C)

Rhynchosporina Arx 1957 (H)

Riclaretia Peyronel 1915 (H)

Rileya A. Funk 1979 (C)

Robakia Petr. 1952 (C)

Robillarda Sacc. 1882 (C)

Rogergoosiella A. Hern. Gut. \& J. Mena 1996 (H)

Roscoepoundia Kuntze 1898 (C)

Rosulomyces S. Marchand \& Cabral 1976 (H)

Rota Bat., Cif. \& Nascim. 1959 (C)

Rotaea Ces. ex Schltdl. 1851 (H)

Rubikia H.C. Evans \& Minter 1985 (C)

Ruggieria Cif. \& Montemart. 1958 (C)

Rutola J.L. Crane \& Schokn. 1978 (H)

Sadasivania Subram. 1957 (H)

Sanjuanomyces R.F. Castañeda \& W.B.

Kendr. 1991 (H)

Santapauinda Subram. 1995 (H)

Saprophragma K.B. Deshp. \& K.S. Deshp. 1966 (H)

Saprotaphrina Verona \& Rambelli 1962

(?C)

Sarbhoyomyces Saikia 1981 (H) 
Sarcinodochium Höhn. 1905 (H)

Sarcinosporon D.S. King \& S.C. Jong 1975 (H)

Sarcopodium Ehrenb. 1818 (H)

Satchmopsis B. Sutton \& Hodges 1975 (C)

Scaphidium Clem. 1901 (C)

Scenomyces F. Stevens 1927 (H)

Sceptrifera Deighton 1965 (H)

Schizoderma Kunze 1825 (C)

Schizothyra Bat. \& C.A.A. Costa 1957 (C)

Schizothyrella Thüm. 1880 (C)

Schizothyropsis Bat. \& A.F. Vital 1960 (C)

Schizotrichum McAlpine 1903 (H)

Schroeteria G. Winter 1881 (H)

Schwarzmannia Pisareva 1968 (C)

Scirrhophoma Petr. 1941 (C)

Sclerococcum Fr. 1825 (H)

Scleroconium Syd. 1935 (H)

Sclerodiscus Pat. 1890 (H)

Sclerodothiorella Died. 1912 (?C)

Sclerographiopsis Deighton 1973 (H)

Sclerographium Berk. 1854 (H)

Scleromeris Syd. 1926 (C)

Scleropycnis Syd. \& P. Syd. 1911 (C)

Sclerotiella A.K. Sarbhoy \& A. Sarbhoy 1975 (H)

Sclerozythia Petch 1937 (C)

Scolecobasidiella M.B. Ellis 1971 (H)

Scolecobasidium E.V. Abbott 1927 (H)

Scolecodochium K. Matsush. \& Matsush. 1996 (H)

Scolecotheca Søchting \& B. Sutton 1997 (C)

Scolecozythia Curzi 1927 (C)

Scoliotidium Bat. \& Cavalc. 1963 (C)

Scopaphoma Dearn. \& House 1925 (C)

Scopulariella Gjaerum 1971 (H)

Scothelius Bat., J.L. Bezerra \& Cavalc. 1965 (C)

Scutisporus K. Ando \& Tubaki 1985 (H)

Scutopeltis Bat. \& H. Maia 1957 (C)

Scutopycnis Bat. 1957 (C)

Seifertia Partr. \& Morgan-Jones 2002 (H)

Seimatosporiopsis B. Sutton, Ghaffer \& Abbas 1972 (C)

Selenodriella R.F. Castañeda \& W.B. Kendr. 1990 (H)

Selenosira Petr. 1957 (C)

Selenosporopsis R.F. Castañeda \& W.B. Kendr. 1991 (H)

Septocyta Petr. 1927 (C)
Mycosphere Doi 10.5943/mycosphere/3/2/5

Septocytella Syd. 1929 (C)

Septogloeum Sacc. 1880 (C)

Septomyxella (Höhn.) Höhn. 1923 (C)

Septopatella Petr. 1925 (C)

Septosporiopsis W.A. Baker \& MorganJones 2009 (H)

Septosporium Corda 1831 (H)

Septotrullula Höhn. 1902 (H)

Sessiliospora D. Hawksw. 1979 (H)

Setolibertella Punith. \& Spooner 1999 (?C)

Setophiale Matsush. 1995 (H)

Setosporella Mustafa \& Abdul-Wahid 1989 (H)

Setosynnema D.E. Shaw \& B. Sutton 1985 (H)

Seychellomyces Matsush. 1981 (H)

Seynesiopsis Henn. 1904 (C)

Shawiella Hansf. 1957 (C)

Sheariella Petr. 1952 (C)

Shrungabeeja V.G. Rao \& K.A. Reddy 1981 (H)

Siamia V. Robert, Decock \& R.F. Castañeda $2000(\mathbf{H})$

Sigmatomyces Sacc. \& P. Syd. 1913 (H)

Sirexcipula Bubák 1907 (C)

Sirocyphis Clem. 1909 (C)

Sirodochiella Höhn. 1925 (H)

Sirogloea Petr. 1923 (C)

Siroligniella Naumov 1926 (C)

Sirophoma Höhn. 1917 (C)

Siroplacodium Petr. 1940 (C)

Siropleura Petr. 1934 (C)

Siroscyphellina Petr. 1923 (C)

Sirosperma Syd. \& P. Syd. 1916 (C)

Sirosphaera Syd. \& P. Syd. 1913 (C)

Sirosporonaemella Naumov 1951 (C)

Sirothecium P. Karst. 1887 (C)

Sirothyriella Höhn. 1910 (C)

Sirothyrium Syd. \& P. Syd. 1916 (C)

Sirozythia Höhn. 1904 (C)

Sirozythiella Höhn. 1909 (C)

Sitochora H.B.P. Upadhyay 1964 (C)

Slimacomyces Minter 1986 (H)

Solicorynespora R.F. Castañeda \& W.B. Kendr. 1990 (H)

Soloacrospora W.B. Kendr. \& R.F. Castañeda 1991 (H)

Solosympodiella Matsush. 1971 (H)

Soloterminospora Matsush. 1996 (H)

Spegazzinia Sacc. 1879 (H)

Speiropsis Tubaki 1958 (H) 
Spermatoloncha Speg. 1908 (H)

Spermochaetella Cif. 1954 (C)

Spermospora R. Sprague 1948 (H)

Spermosporella Deighton 1969 (H)

Sphaeridium Fresen. 1852 (H)

Sphaeriostromella Bubák 1916 (C)

Sphaeriothyrium Bubák 1916 (C)

Sphaeromma H.B.P. Upadhyay 1964 (C)

Sphaeronema Fr. 1815 (?C)

Sphaerophoma Petr. 1924 (C)

Sphaerosporium Schwein. 1832 (H)

Sphaerulomyces Marvanová 1977 (H)

Sphondylocephalum Stalpers 1974 (H)

Spicularia Pers. 1822 (H)

Spinulospora Deighton 1973 (H)

Spiralum J.L. Mulder 1975 (H)

Spiropes Cif. 1955 (H)

Spondylocladiella Linder 1934 (H)

Spondylocladiopsis M.B. Ellis 1963 (H)

Sporidesmiopsis Subram. \& Bhat 1989 (H)

Sporocystis Morgan 1902 (H)

Sporocephalum G. Arnaud 1952 (H)

Sporoglena Sacc. 1894 (H)

Sporophiala P.Rag. Rao 1970 (H)

Sporophora Luteraan 1952 (H)

Stachybotryella Ellis \& Barthol. 1902 (H)

Stagonopatella Petr. 1927 (C)

Stagonopsis Sacc. 1884 (C)

Stagonosporina Tassi 1902 (C)

Stagonostromella Petr. \& Syd. 1927 (C)

Staheliella Emden 1974 (H)

Stalagmochaetia Cif. \& Bat. 1963 (C)

Staphylotrichum J. Mey. \& Nicot 1957 (H)

Stauronema (Sacc.) Syd., P. Syd. \& E.J. Butler 1916 (C)

Stauronematopsis Abbas, B. Sutton \& Ghaffar 2002 (C)

Staurophoma Höhn. 1907 (C)

Stegolerium Strobel, W.M. Hess \& E.J. Ford 2001 (H)

Stegonsporiopsis Van Warmelo \& B. Sutton 1981 (C)

Stellomyces Morgan-Jones, R.C. Sinclair \& Eicker 1987 (H)

Stellopeltis Bat. \& A.F. Vital 1959 (C)

Stellospora Alcorn \& B. Sutton 1984 (H)

Stellothyriella Bat. \& Cif. 1959 (C)

Stemphyliomma Sacc. \& Traverso 1911 (H)

Stenocephalopsis Chamuris \& C.J.K. Wang 1998 (H)
Stenocladiella Marvanová \& Descals 1987 (H)

Stenospora Deighton 1969 (H)

Stephembruneria R.F. Castañeda 1988 (H)

Stevensonula Petr. 1952 (C)

Stichospora Petr. 1927 (C)

Stictopatella Höhn. 1918 (C)

Stictosepta Petr. 1964 (C)

Stigmatellina Bat. \& H. Maia 1960 (C)

Stigmella Lév. 1842 (C)

Stigmopeltis Syd. 1927 (C)

Stilbellula Boedijn 1951 (H)

Stilbophoma Petr. 1942 (C)

Stilbospora Pers. 1794, also see Prosthecium $\mathbf{( C )}$

Strasseria Bres. \& Sacc. 1902 (C)

Strasseriopsis B. Sutton \& Tak. Kobay. 1970 (C)

Stratiphoromyces Goh \& K.D. Hyde 1998

(H)

Striosphaeropsis Verkley \& Aa 1997 (C)

Stromatopogon Zahlbr. 1897 (C)

Stromatopycnis A.F. Vital 1956 (C)

Stromatostysanus Höhn. 1919 (H)

Strongylothallus Bat. \& Cif. 1959 (H)

Strumellopsis Höhn. 1909 (H)

Stygiomyces Coppins \& S.Y. Kondr. 1995

(C)

Stylaspergillus B. Sutton, Alcorn \& P.J. Fisher 1982 (H)

Subicularium M.L. Farr \& Goos 1989 (H)

Subramania D. Rao \& P.Rag. Rao 1964 (H)

Subramanianospora Narayanan, J.K. Sharma \& Minter 2003 (H)

Subulispora Tubaki 1971 (H)

Sutravarana Subram. \& Chandrash. 1977 (H)

Suttoniella S. Ahmad 1961(C)

Suttonina H.C. Evans 1984 (C)

Syamithabeeja Subram. \& Natarajan 1976 (H)

Sylviacollaea Cif. 1963 (C)

Symbiotaphrina Kühlw. \& Jurzitza ex W. Gams \& Arx 1980 (H)

Symphyosira Preuss 1853 (H)

Symphysos Bat. \& Cavalc. 1967 (C)

Sympodiocladium Descals 1982 (H)

Sympodioclathra Voglmayr 1997 (H)

Sympodioplanus R.C. Sinclair \& Boshoff 1997 (H) 
Synchronoblastia Uecker \& F.L. Caruso 1988 (C)

Syncladium Rabenh. 1859 (C)

Synnemacrodictys W.A. Baker \& MorganJones 2009 (H)

Synnemaseimatoides K. Matsush. \& Matsush. 1996 (H)

Synnematium Speare 1920 (H)

Synnematomyces Kobayasi 1981 (H)

Synnemellisia N.K. Rao, Manohar. \& Goos 1989 (H)

Synostomina Petr. 1949 (C)

Systremmopsis Petr. 1923 (H)

Taeniolina M.B. Ellis 1976 (H)

Talekpea Lunghini \& Rambelli 1979 (H)

Tandonia M.D. Mehrotra 1991 (C)

Tandonella S.S. Prasad \& R.A.B. Verma 1970 (H)

Tarsodisporus Bat. \& A.A. Silva 1965 (C)

Tawdiella K.B. Deshp. \& K.S. Deshp. 1966 (H)

Tectacervulus A.W. Ramaley 1992 (C)

Telligia Hendr. 1948 (H)

Temerariomyces B. Sutton 1993 (H)

Teratosperma Syd. \& P. Syd. 1909 (H)

Tetrabrachium Nawawi \& Kuthub. 1987 (H)

Tetrabrunneospora Dyko 1978 (H)

Tetracoccosporium Szabó 1905 (H)

Tetranacriella Kohlm. \& Volkm.-Kohlm. 2001 (?C)

Tetranacrium H.J. Huds. \& B. Sutton 1964 (C)

Tetraposporium S. Hughes 1951 (H)

Textotheca Matsush. 1996 (C)

Thallospora L.S. Olive 1948 (H)

Thaptospora B. Sutton \& Pascoe 1987 (C)

Tharoopama Subram. 1956 (H)

Thirumalacharia Rathaiah 1981 (H)

Tholomyces Matsush. 2003 (?C)

Thoracella Oudem. 1900 (C)

Thrinacospora Petr. 1948 (C)

Thyriostromella Bat. \& C.A.A. Costa 1959 (C)

Thyrostromella Höhn. 1919 (H)

Thyrsidiella Höhn. ex Höhn. 1909 (?C)

Thyrsidina Höhn. 1905 (C)

Tiarosporellivora Punith. 1981

Ticogloea G. Weber, Spaaij \& W. Gams 1994 (H)

Tilakiopsis V.G. Rao 1994 (H)

Titaeopsis B. Sutton \& Deighton 1984 (H)
Mycosphere Doi 10.5943/mycosphere/3/2/5

Titaeospora Bubák 1916, also see Stamnaria $\mathbf{( C )}$

Tomenticola Deighton 1969 (H)

Tompetchia Subram. 1985 (H)

Toxosporiella B. Sutton 1986 (C)

Toxosporiopsis B. Sutton \& Sellar 1966 (C)

Toxosporium Vuill. 1896 (H)

Tracylla (Sacc.) Tassi 1904 (C)

Trematophoma Petr. 1924 (C)

Tremellidium Petr. 1927 (C)

Tretendophragmia Subram. 1995 (H)

Tretocephala Subram. 1995 (H)

Tretophragmia Subram. \& Natarajan 1974 (H)

Tretospeira Piroz. 1972 (H)

Tretovularia Deighton 1984 (H)

Triadelphia Shearer \& J.L. Crane 1971

(H)

Tribolospora D.A. Reid 1966 (C)

Trichaegum Corda 1837 (H)

Trichobolbus Bat. 1964 (C)

Trichobotrys Penz. \& Sacc. 1901 (H)

Trichoconiella B.L. Jain 1976 (H)

Trichoconis Clem. 1909 (H)

Trichodiscula Vouaux 1910 (C)

Trichodochium Syd. 1927 (H)

Trichomatoclava G.F. Sepúlveda, PereiraCarv. \& Dianese 2009 (H)

Trichomatomyces Dorn.-Silva \& Dianese 2004 (H)

Trichomatosphaera Pereira-Carv., G.F. Sepúlveda \& Dianese 2009 (H)

Trichopeltulum Speg. 1889 (C)

Trichoseptoria Cavara 1892 (C)

Trichosporodochium Dorn.-Silva \& Dianese 2004 (H)

Trichotheca P. Karst. 1887 (H)

Tricladiella K. Ando \& Tubaki 1984 (H)

Tricladiopsis Descals 1982 (H)

Tricladiospora Nawawi \& Kuthub. 1988 (H)

Tricornispora Bonar 1967 (H)

Trifurcospora K. Ando \& Tubaki 1988 (H)

Triglyphium Fresen. 1852 (H)

Trigonosporium Tassi 1900 (C)

Tripoconidium Subram. 1978 (H)

Triposporina Höhn. 1912 (H)

Triramulispora Matsush. 1975 (H)

Triscelophorus Ingold 1944 (H)

Triscelosporium Nawawi \& Kuthub. 1987 
(H)

Trisulcosporium H.J. Huds. \& B. Sutton 1964 (H)

Troposporium Harkn. 1884 (H)

Troposporopsis Whitton, McKenzie \& K.D. Hyde 1999 (H)

Trullula Ces. 1852 (C)

Tryssglobulus B. Sutton \& Pascoe 1987 (H)

Tuberculariopsis Höhn. 1909 (H)

Tuberculispora Deighton \& Piroz. 1972 (H)

Tulipispora Révay \& Gönczöl 2009 (H)

Tunicago B. Sutton \& Pollack 1977 (C)

Turturconchata J.L. Chen, T.L. Huang \& Tzean 1999 (H)

Tympanosporium W. Gams 1974 (H)

Uberispora Piroz. \& Hodges 1973 (H)

Ubrizsya Negru 1965 (C)

Ulocoryphus Michaelides, L. Hunter \& W.B. Kendr. 1982 (H)

Umbellidion B. Sutton \& Hodges 1975 (H)

Uncispora R.C. Sinclair \& Morgan-Jones 1979 (H)

Urohendersonia Speg. 1902 (C)

Urohendersoniella Petr. 1955 (C)

Uvarispora Goos \& Piroz. 1975 (H)

Vagnia D. Hawksw. \& Miądl. 1997 (C)

Vanakripa Bhat, W.B. Kendr. \& Nag Raj 1993 (H)

Vanbeverwijkia Agnihothr. 1961 (H)

Vanderystiella Henn. 1908 (C)

?Vanibandha Manohar., N.K. Rao,

Kunwar \& D.K. Agarwal 2006 (H)

Vanterpoolia A. Funk 1982 (H)

Variocladium Descals \& Marvanová 1999 (H)

Vasudevella Chona, Munjal \& Bajaj 1957

(C)

Velutipila D. Hawksw. 1987 (H)

Ventrographium H.P. Upadhyay, Cavalc. \& A.A. Silva 1986 (H)

Venustocephala Matsush. 1995(H)

Venustosynnema R.F. Castañeda \& W.B. Kendr. 1990 (H)

Veracruzomyces Mercado, Guarro, Heredia \& J. Mena $2002(\mathbf{H})$

Veramycella G. Delgado 2009 (H)

Veramyces Matsush. 1993 (H)

Verdipulvinus A.W. Ramaley 1999 (H)

Vermispora Deighton \& Piroz. 1972 (H)

Veronaella Subram. \& K.R.C. Reddy 1975
Veronidia Negru 1964 (C)

Verrucariella S. Ahmad 1967 (C)

Verrucophragmia Crous, M.J. Wingf. \&

W.B. Kendr. 1994 (H)

Verticicladus Matsush. 1993 (H)

Vesiculohyphomyces Armando, Pereira-

Carv. \& Dianese 2009 (H)

Vestigium Piroz. \& Shoemaker 1972 (C)

Viscomacula R. Sprague 1951 (H)

Vittalia Gaws \& Bhat 2007 (H)

Vouauxiella Petr. \& Syd. 1927 (C)

Waihonghopes Yanna \& K.D. Hyde 2002

(H)

Wardinella Bat. \& Peres 1960 (C)

Waydora B. Sutton 1976 (C)

Websteromyces W.A. Baker \& Partr. 2000 (H)

Weissia Bat. \& M.P. Herrera 1964 (C)

Weufia Bhat \& B. Sutton 1985 (H)

Wiesneriomyces Koord. 1907 (H)

Wilsonomyces Adask., J.M. Ogawa \& E.E.

Butler 1990 (H)

Wojnowicia Sacc. 1899 (C)

Xanthoriicola D. Hawksw. 1973 (H)

Xenidiocercus Nag Raj 1993 (C)

Xenodomus Petr. 1922 (C)

Xenoheteroconium Bhat, W.B. Kendr. \& Nag Raj 1993 (H)

Xenokylindria DiCosmo, S.M. Berch \& W.B. Kendr. 1983 (H)

Xenopeltis Syd. \& P. Syd. 1919 (C)

Xenoplaca Petr. 1949 (H)

Xenostroma Höhn. 1915 (C)

Xepicula Nag Raj 1993 (C)

Xepiculopsis Nag Raj 1993 (C)

Xeroconium D. Hawksw. 1981 (C)

Xiambola Minter \& Hol.-Jech. 1981 (H)

Xiphomyces Syd. \& P. Syd. 1916 (H)

Xylochia B. Sutton 1983 (H)

Xyloglyphis Clem. 1909 (C)

Xylohypha (Fr.) E.W. Mason 1960 (H)

Xylohyphopsis W.A. Baker \& Partr. 2000

(H)

Yalomyces Nag Raj 1993 (C)

Yinmingella Goh, K.M. Tsui \& K.D. Hyde 1999 (H)

Ypsilina J. Webster, Descals \& Marvanová 1999 (H)

Yuccamyces Gour, Dyko \& B. Sutton 1979

(H)

Zakatoshia B. Sutton 1973 (H)

Zanclospora S. Hughes \& W.B. Kendr. 
1965 (H)

Zebrospora McKenzie 1991 (H)

Zelandiocoela Nag Raj 1993 (C)

Zeloasperisporium Castaneda 1996 (H)

Zelopelta B. Sutton \& R.D. Gaur 1984 (C)

Zelosatchmopsis Nag Raj 1991 (C)

Zelotriadelphia R.F. Castañeda, Saikawa, M. Stadler \& Iturr. 2005 (H)

Zetesimomyces Nag Raj 1988 (C)

Zevadia J.C. David \& D. Hawksw. 1995 (H)

Zilingia Petr. 1934 (C)

Zinzipegasa Nag Raj 1993 (C)

Zopheromyces B. Sutton \& Hodges 1977 (H)

Zunura Nag Raj 1993 (C)

Zygosporium Mont. 1842 (H)

Zythia Fr. 1849 (C)

Zyxiphora B. Sutton 1981 (H)

Phylum BASIDIOMYCOTA R.T. Moore Subphylum AGARICOMYCOTINA Doweld Class Agaricomycetes Doweld

Agaricales Underw.

Agaricaceae Chevall.

Coccobotrys Boud. \& Pat. 1900, anamorphic Leucoagaricus Locq. ex Singer 1948 (H)

Fistulinaceae Lotsy

Confistulina Stalpers 1983, anamorphic Fistulina Bull. 1791 (H)

\section{Lyophyllaceae Jülich}

Termitosphaera Cif. 1935, anamorphic Termitomyces R. Heim 1942 (H)

Ugola Adans. 1763, anamorphic Asterophora Ditmar 1809 (H)

\section{Mycenaceae Overeem}

Decapitatus Redhead \& Seifert 2000, anamorphic Mycena (Pers.) Roussel $1806(\mathbf{H})$

Niaceae Jülich

Peyronelina P.J. Fisher, J. Webster \& D.F. Kane 1976 (H)

\section{Pleurotaceae Kühner}

Antromycopsis Pat. \& Trab. 1897, anamorphic Pleurotus (Fr.) P. Kumm. $1871(\mathbf{H})$
Mycosphere Doi 10.5943/mycosphere/3/2/5

Nematoctonus Drechsler 1941, anamorphic Hohenbuehelia Schulzer 1866 (H)

Psathyrellaceae Vilgalys, Moncalvo \& Redhead

Hormographiella Guarro \& Gené 1992, Coprinellus P. Karst. 1879 (H)

Rhacophyllus Berk. \& Broome 1871, anamorphic Coprinopsis P. Karst. 1881 (H)

Tricholomataceae R. Heim ex Pouzar

Moniliophthora H.C. Evans, Stalpers, Samson \& Benny 1978 (H)

Nothoclavulina Singer 1970, anamorphic Arthrosporella Singer 1970 (H)

Tilachlidiopsis Keissl. 1924, anamorphic Dendrocollybia R.H. Petersen \& Redhead 2001 (H)

Ugola Adans. 1763(H)

Tilachlidiopsis Keissl. 192 (H)

Typhulaceae Jülich

Sclerotium Tode 1790, anamorphic Typhula (Pers.) Fr. 1818 (H)

Agaricales, genera incertae sedis

Disporotrichum Stalpers 1984

Fibulochlamys A.I. Romero \& Cabral 1989 (H)

Tricladiomyces Nawawi 1985 (H)

Atheliales Jülich

Atheliaceae Jülich

Fibularhizoctonia G.C. Adams \& Kropp 1996, anamorphic Athelia Pers. 1822 (H)

Rhizoctonia DC. 1815 anamorphic Athelia Pers. $1822(\mathbf{H})$

Sclerotium Tode 1790, anamorphic Athelia Pers. $1822(\mathbf{H})$

Taeniospora Marvanová 1977, anamorphic Fibulomyces Jülich 1972 (H)

Auriculariales J. Schröt. 1897

Exidiaceae R.T. Moore 1978

Ovipoculum Zhu L. Yang \& R. Kirschner $2010(\mathbf{H})$

Auriculariales, genera incertae sedis

Disporotrichum Stalpers 1984 (H)

Oliveorhiza P. Roberts 1998, anamorphic Oliv-eonia Donk 1958 (H) 
Mycosphere Doi 10.5943/mycosphere/3/2/5

Cantharellales Gäum

Botryobasidiaceae Jülich

Haplotrichum Link 1824, anamorphic Botryobasidium Donk 1931 (H)

Ceratobasidiaceae G.W. Martin

Acanthellorhiza P. Roberts 1999, anamorphic Heteroacanthella 1990 (H)

Ceratorhiza R.T. Moore 1987, anamorphic Ceratobasidium D.P. Rogers 1935 (H)

Rhizoctonia DC. 1815 (and as Thanatophytum Nees 1816), anamorphic Helicobasidium Pat. 1885, Aquathanatephorus C.C. Tu \& Kimbr. 1978 and Thanatephorus Donk 1956 (H)

Hydnaceae Chevall.

Burgella Diederich \& Lawrey 2007 (H)

Burgoa Goid. 1938, anamorphic Sistotrema Fr. 1821 (H)

Ingoldiella D.E. Shaw 1972, anamorphic Sistotrema Fr. 1821 (H)

Osteomorpha G. Arnaud ex Watling \& W.B. Kendr. 1979, anamorphic Sistotrema Fr. 1821 and Trechispora P. Karst. 1890 (H)

Oliveoniaceae P. Roberts 1998

Oliveorhiza P. Roberts 1998, anamorphic Oliveonia Donk 1958 (H)

Tulasnellaceae Juel

Epulorhiza R.T. Moore 1987, anamorphic Tulasnella J. Schröt. 1888 (H)

Cantharellales, genera incertae sedis

Minimedusa Weresub \& P.M. LeClair 1971 (H)

Corticiales K.H. Larss.

Corticiaceae Herter

Chrysorhiza T.F. Andersen \& Stalpers 1996, anamorphic Waitea Warcup \& P.H.B. Talbot 1962

Erythricium J. Erikss. \& Hjortstam 1970

Marchandiomyces Dieder. \& D. Hawksw. 1990, anamorphic Marchandiobasidium Diederich \& Schultheis 2003 (H)

Michenera Berk. \& M.A. Curtis 1868, anamorphic Licrostroma P.A. Lemke 1964 (H)

Tretopileus B.O. Dodge 1946 (H)
Hymenochaetales Oberw.

Schizoporaceae Jülich

Echinodia Pat. 1918, anamorphic Echinoporia Ryvarden 1980 (H)

Hymenochaetales, genera incertae sedis

Caeruleomyces Stalpers 2000

Polyporales Gäum.

Corticiaceae Herter

Necator Massee 1898 (H)

Fomitopsidaceae Jülich

Ptychogaster Corda 1838, anamorphic Oligoporus Bref. 1888 and Postia Fr. 1874 (H)

Sporotrichum Link 1809, anamorphic Laetiporus Murrill 1904 and Pycnoporellus Murrill 1905 (H)

Ganodermataceae Donk

Thermophymatospora Udagawa, Awao \& Abdullah 1986, anamorphic Ganoderma P. Karst. 1881 (H)

Hyphodermataceae Jülich

Aegerita Pers. 1801, anamorphic Bulbillomyces Jülich 1974 (H)

Aegeritina Jülich 1984, anamorphic Subulicystidium Parmasto 1968 (H)

Meruliaceae P. Karst.

Bornetina L. Mangin \& Viala 1903, anamorphic Diacanthodes Singer 1945 (H)

Sporotrichopsis Staplers (2000), anamorphic Abortiporus Murrill (H)

Phanerochaetaceae Jülich

Erythricium J. Erikss. \& Hjortstam 1970, anamorph of Phanerochaete P. Karst. 1889

Sporotrichum Link 1809, anamorphic Phanerochaete P. Karst. 1889 (H)

Polyporaceae Fr. ex Corda

Digitellus Paulet 1791, anamorphic Lentinus Fr. 1825 (H)

Mycelithe Gasp. 1841, anamorphic Polyporus P. Micheli ex Adans. 1763 (C)?

Pachyma Fr. 1822, anamorphic Lentinus Fr. 1825, Macrohyporia I. Johans. \& 
Mycosphere Doi 10.5943/mycosphere/3/2/5

Ryvarden 1979, Polyporus P. Micheli ex Adans. 1763, Lignosus Lloyd ex Torrend 1920 and Wolfiporia Ryvarden \& Gilb. 1984 (H)

Russulales Kreisel ex P.M. Kirk, P.F. Cannon \& J.C. David

Bondarzewiaceae Kotl. \& Pouzar

Spiniger Stalpers 1974, anamorphic Bondarzewia Singer 1940 and Heterobasidion Bref. 1888 (H)

Echinodontiaceae Donk

Spiniger Stalpers 1974, anamorphic Laurilia Pouzar 1959 (H)

Lachnocladiaceae D.A. Reid

Spiniger Stalpers 1974, anamorphic Dichostereum Pilát 1926 (H)

\section{Stereaceae Pilát}

Acaromyces Boekhout, Scorzetti, Gerson \& Sztejnb. 2003 (H)

Aleurocystis Lloyd ex G. Cunn. 1956, anamorphic Licrostroma P.A. Lemke 1964 (?C)

Matula Massee 1888, anamorphic Aleurocystis Lloyd ex G. Cunn.1956 (H)

Sebacinales M. Weiss, Selosse, Rexer, A. Urb. \& Oberw.

Sebacinaceae K. Wells \& Oberw.

Craterocolla Bref. 1888

Ditangium P. Karst. 1867, anamorphic Craterocolla Bref. 1888 (H)

?Chaetospermum Sacc. 1892, anamorphic Efibulobasidium K. Wells 1975 (C)

Flahaultiella Seifert 2009, anamorphic Sebacina Tul. \& C. Tul. 1871 (H)

Opadorhiza T.F. Andersen \& R.T. Moore 1996, anamorphic Sebacina Tul. \& C. Tul. 1871 (H)

Piriformospora Sav. Verma, Aj. Varma, Rexer, G. Kost \& P. Franken 1998 (H)

Thelephorales Corner ex Oberw.

Thelephoraceae Chevall.

Parahaplotrichum W.A. Baker \& Partr. 2001, anamorphic Botryobasidium Rick 1959 (H)

Agaricomycetes, genera incertae sedis
Akenomyces G. Arnaud ex D. Hornby 1984 (H)

Arthrodochium R.F. Castañeda \& W.B. Kendr. 1990 (H)

Arualis Katz 1980 (H)

Bartheletia G. Arnaud ex Scheuer, R. Bauer, M. Lutz, Stabenth., Melnik \& Grube 2008 (H)

Cenangiomyces Dyko \& B. Sutton 1979 (H)

Cruciger R. Kirschner \& Oberw. 1999 (H)

Dendrosporomyces Nawawi, J. Webster \& R.A. Davey 1977 (H)

Ellula Nag Raj 1980 (C)

Fibulocoela Nag Raj 1978 (C)

Fibulotaeniella Marvanová \& Bärl. 1988 (H)

Geotrichopsis Tzean \& Estey 1991 (H)

Gloeosynnema Seifert \& G. Okada 1988 (H)

Glomerulomyces A.I. Romero \& S.E. López 1989 (H)

Glutinoagger Sivan. \& Watling 1980 (H)

Myriococcum Fr. 1823 (H)

Nyctalina G. Arnaud 1952 (H)

Pagidospora Drechsler 1960 (H)

Pycnovellomyces R.F. Castañeda 1987 (C)

Riessia Fresen. 1852 (H)

Riessiella Jülich 1985 (H)

Titaeella G. Arnaud ex K. Ando \& Tubaki 1985 (H)

Tricladiomyces Nawawi 1985 (H)

Class Dacrymycetes Doweld

Dacrymycetales Henn.

Dacrymycetaceae J. Schröt.

Cerinosterus R.T. Moore 1987 (H)

Dacryoscyphus R. Kirschner \& Zhu L. Yang 2005 (H)

Class Tremellomycetes Hibbett, Matheny, \& Manfr. Binder

Cystofilobasidiales Fell, Roeijmans \& Boekhout

Cystofilobasidiaceae K. Wells \& Bandoni Guehomyces Fell \& Scorzetti 2004 Itersonilia Derx 1948

Mrakiella Margesin \& Fell 2008 (H) Phaffia M.W. Mill., Yoney. \& Soneda 1976, anamorphic Xanthophyllomyces Golubev 1995 (C) 
Rhodozyma Phaff, M.W. Mill., Yoney. \& Soneda 1972, anamorphic Xanthophyllomyces Golubev 1995

Tausonia Babeva 1998

Udeniomyces Nakase \& Takem. 1992 (Y)

Filobasidiales Jülich

Filobasidiaceae L.S. Olive

Cryptococcus Vuill. 1901, anamorphic Filobasidium L.S. Olive 1968 (Y)

Tremellales Fr.

Cuniculitremaceae J.P. Samp., R. Kirschner \& M. Weiss

Fellomyces Y. Yamada \& I. Banno 1984 (Y)

Kockovaella Nakase, I. Banno \& Y. Yamada 1991 (Y)

Sterigmatosporidium G. Kraep. \& U. Schulze 1983, anamorphic Cuniculitrema J.P. Samp. \& R. Kirschner 2001 (Y)

Tremellina Bandoni 1986, anamorphic Cuniculitrema J.P. Samp. \& R. Kirschner 2001 (H)

Hyaloriaceae Lindau

Helicomyxa R. Kirschner \& Chee J. Chen 2004 (H)

\section{Tremellaceae Fr.}

Anastomyces W.P. Wu, B. Sutton \& Gange 1997 (H)

Bullera Derx 1930 (Yeast)

Cryptococcus Vuill. 1901, anamorphic Filobasidiella Kwon-Chung 1976 (Y)

Hormomyces Bonord. 1851, anamorphic Tremella Pers. 1794 (H)

Hormomyces-like anamorphic Biatoropsis Räsänen 1934 (H)

Tsuchiyaea Y. Yamada, H. Kawas., Itoh, I. Banno \& Nakase 1988 (H)

Trichosporonaceae Nann.

Asterotremella Prillinger, Lopandic \& Sugita 2007 (Y)

Trichosporon Behrend 1890 (Y)

Tritirachium Limber 1940, anamorphic Neorehmia Höhn. 1902 (H)

Tremellales genera incertae cedis
Mycosphere Doi 10.5943/mycosphere/3/2/5

Derxomyces F.Y. Bai \& Q.M. Wang 2008 (Y)

Hannaella F.Y. Bai \& Q.M. Wang 2008 (Y)

Moniliella Stolk \& Dakin 1966 (H)

Tremellomycetes, genera incertae sedis

Heteromycophaga P. Roberts 1997 (Yeastlike)

Moniliella Stolk \& Dakin 1966 (H)

Trichosporonoides Haskins \& J.F.T. Spencer 1967 (H)

AGARICOMYCOTINA, genera incertae sedis Microstella K. Ando \& Tubaki 1984 (H)

Subphylum PUCCINIOMYCOTINA R. Bauer, Begerow, J.P. Samp., M. Weiss \& Oberw

Class Agaricostilbomycetes R. Bauer, Begerow, J.P. Samp., M. Weiss \& Oberw.

Agaricostilbales Oberw. \& R. Bauer

Agaricostilbaceae Oberw. \& R. Bauer

Agaricostilbum J. Wright 1970 (H)

Bensingtonia Ingold 1986 (Y)

Sterigmatomyces Fell 1966 (Y)

Chionosphaeraceae Oberw. \& Bandoni

Kurtzmanomyces Y. Yamada, Itoh, H.

Kawas., I. Banno \& Nakase 1989 (Y) Mycogloea L.S.Olive 1950 (Y)

Class Atractiellomycetes R. Bauer, Begerow, J.P. Samp., M. Weiss \& Oberw.

Atractiellales Oberw. \& Bandoni

Phleogenaceae Gäum.

Atractiella Sacc. 1886 (H)

Basidiopycnides J. Reid, Eyjólfsd. \& Georg Hausner 2008, anamorphic Basidiopycnis Oberw., R. Kirschner, R.Bauer, Begerow \& Arenal 2006 (H)

Saccoblastiaceae Jülich

Infundibura Nag Raj \& W.B. Kendr. 1981

(H)

Atractiellales, genera incertae sedis

Atractogloea Oberw. \& Bandoni 1982 (H)

Hobsonia Berk. ex Massee 1891 (H)

Leucogloea R. Kirschner 2004 (H)

Tricladiomyces Nawawi 1985 (H) 
Pachnocybales R. Bauer, Begerow, J.P. Samp., M. Weiss \& Oberw. 2006

Pachnocybaceae Oberw. \& R. Bauer 1989 Pachnocybe Berk. 1836 (H)

Class Classiculomycetes R. Bauer, Begerow, J.P. Samp., M. Weiss \& Oberw.

Classiculales R. Bauer, Begerow, Oberw. \& Marvanová

Classiculaceae Bauer, Begerow, Oberw. \& Marvanová

Jaculispora H.J. Huds. \& Ingold 1960 (H)

Naiadella Marvanová \& Bandoni 1987, anamorphic Classicula R. Bauer, Begerow, Oberw. \& Marvanová 2003 (H)

Class Cryptomycocolacomycetes R. Bauer, Begerow, J.P. Samp., M. Weiss \& Oberw.

Cryptomycocolacales Oberw. \& R. Bauer Cryptomycocolacaceae Oberw. \& R. Bauer

Colacosiphon R. Kirschner, R. Bauer \& Oberw. 2001 (H)

Class Cystobasidiomycetes R. Bauer, Begerow, J.P. Samp., M. Weiss \& Oberw.

Erythrobasidiales, genera incertae sedis

Bannoa Hamam. 2002 (Y)

Erythrobasidium Hamam., Sugiy. \& Komag. 1988 (Y)

Class Microbotryomycetes R. Bauer, Begerow, J.P. Samp., M. Weiss \& Oberw.

Heterogastridiales Oberw. \& R. Bauer

Heterogastridiaceae Oberw. \& R. Bauer Hyalopycnis Höhn. 1918, anamorphic Heterogastridium Oberw. \& R. Bauer 1990 (C)

Leucosporidiales J.P. Samp., M. Weiss \& R. Bauer

Leucosporidiaceae Jülich Leucosporidiella Samp. 2003 (Y)

Microbotryales R. Bauer \& Oberw., genera incertae sedis

Reniforma Pore \& Sorenson 1990 (Y)

Sporidiobolales Doweld

Sporidiobolaceae R.T. Moore

Blastoderma B. Fisch. \& Brebeck 1894, anamorphic Sporidiobolus Nyland 1950 (Y)
Rhodomyces Wettst. 1885, anamorphic Sporidiobolus Nyland 1950

Sporobolomyces Kluyver \& C.B. Niel 1924, anamorphic Sporidiobolus Nyland 1950 (H)

Sporidiobolales, genera incertae cedis

Ballistosporomyces Nakase, G. Okada \& Sugiy., in Nakase, Okada, Sugiyama, Itoh \& Suzuki 1989

Erythrobasidium Hamam., Sugiy. \& Komag. 1988, anamorphic Rhodosporidium Banno 1967 (Y)

Rhodotorula F.C. Harrison 1927, anamorphic Rhodosporidium Banno 1967 (Y)

Microbotryomycetes, genera incertae sedis

Crucella Marvanová \& Suberkr. 1990, anamorphic Camptobasidium Marvanová \& Suberkr. 1990 (H)

Class Pucciniomycetes R. Bauer, Begerow, J.P. Samp., M. Weiss \& Oberw.

Helicobasidiales R. Bauer, Begerow, J.P. Samp., M. Weiss \& Oberw.

Helicobasidiaceae P.M. Kirk

Thanatophytum Nees 1816, anamorphic Helicobasidium Pat. 1885 (H)

Tuberculina Tode ex Sacc. 1880, anamorphic Helicobasidium Pat. 1885 (H)

Platygloeales R.T. Moore

Eocronartiaceae Jülich

Glomopsis D.M. Hend. 1961, anamorphic Herpobasidium Lind 1908 (H)

Platygloeaceae Racib. 1909

Infundibura Nag Raj \& W.B. Kendr. 1981, anamorphic Helicogloea Pat. 1892 (H)

Leucogloea R. Kirschner 2004 anamorphic Helicogloea Pat. 1892 (H)

Uncolaceae Buriticá

Calidion Syd. \& P. Syd. 1919 (R)

Pucciniales Clem. \& Shear

Coleosporiaceae Dietel

?Chrysomyxa Unger 1840, anamorphic Peridermium (Link) J.C. Schmidt \& Kunze 1817 (R) 
Cronartiaceae Dietel

?Cronartium Fr. 1815, anamorphic Peridermium (Link) J.C. Schmidt \& Kunze 1817 (R)

Phakopsoraceae Cummins \& Hirats. f.

Aeciure Buriticá \& J.F. Hennen 1994, anamorphic Arthuria H.S. Jacks (R)

Macabuna Buriticá \& J.F. Hennen 1994

Malupa Y. Ono, Buriticá \& J.F. Hennen 1992 (R)

Milesia F.B. White 1878, anamorphic Phakopsora Dietel 1895 but see Milesina Magnus 1909 (R)

Physopella Arthur 1906, anamorphic Cerotelium Arthur 1906 and Phakopsora Dietel 1895 (R)

Uredendo Buriticá \& J.F. Hennen 1994, anamorphic Phakopsora Dietel 1895 (?R)

Uredostilbe Buriticá \& J.F. Hennen 1994 (?R)

\section{Phragmidiaceae Corda}

Gerwasia Racib. 1909, anamorphic Campanulospora Salazar-Yepes, PardoCard. \& Buriticá 2007, Morispora Salazar-Yepes, Pardo-Card. \& Buriticá 2007 and Scutelliformis Salazar-Yepes, Pardo-Card. \& Buriticá 2007 (R)

Physonema Lév. 1847, anamorphic Phragmidium Link 1815 (?R)

Puccineaceae Chevall.

Caeoma Link 1809 (R)

Roestelia Rebent. 1804, anamorphic Gymnosporangium R. Hedw. ex DC. 1805 (R)

Pucciniastraceae Gäum. ex Leppik

Milesia F.B. White 1878, anamorphic Milesina Magnus 1909, but see Phakopsora Dietel 1895 (R)

Peridiopsora Kamat \& Sathe 1969, anamorphic Milesina Magnus 1909 (R)

Pomatomyces Oerst. 1864, anamorphic Thekopsora Magnus 1875 (?R)

Pucciniastrum G.H. Otth 1861, ?anamorphic Peridermium (Link) J.C. Schmidt \& Kunze 1817 (R)

Uropyxidaceae Cummins \& Y. Hirats.
Canasta A.A. Carvalho \& J.F. Hennen 2010, anamorphic Prospodium Arthur 1907 (R)

Pucciniales, genera incertae sedis

Elateraecium Thirum., F. Kern \& B.V. Patil 1966, anamorphic Hiratsukamyces

Thirum., F. Kern \& B.V. Patil 1975 (R)

Intrapes J.F. Hennen \& Figueiredo 1979 (?R)

Uraecium Arthur 1933 (R)

Uredo Pers. 1801 (R)

Septobasidiales Couch ex Donk

Septobasidiaceae Racib.

Johncouchia S. Hughes \& Cavalc. 1983, anamorphic Septobasidium Pat. 1892 (H)

Subphylum USTILAGINOMYCOTINA R. Bauer, Begerow, J.P. Samp., M. Weiss \& Oberw.

Class Exobasidiomycetes Begerow, M. Stoll, R. Bauer

Doassansiales R. Bauer \& Oberw.

Doassansiaceae R.T. Moore ex P.M. Kirk, P.F. Cannon \& J.C. David

Savulescuella Cif. 1959, anamorphic Doassansia Cornu 1883 (H)

Entylomatales R. Bauer \& Oberw.

Entylomataceae R. Bauer \& Oberw.

Entylomella Höhn. 1924, anamorphic Entyloma de Bary 1874 (H)

Tilletiopsis Derx 1948, anamorphic Entyloma de Bary 1874 and Melanotaenium de Bary 1874 (H)

Exobasidiales Henn.

Cladostergigma Pat. 1892 (H)

Clinoconidium Pat. 1898 (H)

Coniodictyum Har. \& Pat. 1909 (H)

Brachybasidiaceae Gäum.

Meira Boekhout, Scorzetti, Gerson \& Sztejnb. 2003 (H)

Microstromatales R. Bauer \& Oberw.

Microstromataceae Jülich Microstroma Niessl 1861 (H)

Sympodiomycopsis Sugiy., Tokuoka \& 
Mycosphere Doi 10.5943/mycosphere/3/2/5

Komag. 1991 (?Y)

Microstromatales, genera incertae sedis Quambalaria J.A. Simpson 2000 (H)

Class Ustilaginomycetes R. Bauer, Oberw. \& Vánky

Urocystidales R. Bauer \& Oberw.

Glomosporiaceae Cif.

Rhombiella Liro 1939, anamorphic Thecaphora Fingerh. 1836 and Tothiella Vánky 1999 (H)

Thecaphorella H. Scholz \& I. Scholz 1988, anamorphic Thecaphora Fingerh. $1836(\mathbf{H})$

Ustilaginales G. Winter

Anthracoideaceae Denchev

Crotalia Liro 1938, anamorphic Anthracoidea Bref. 1895 (?Y)

\section{Cintractiellaceae Vánky}

Naiadella Marvanová \& Bandoni 1987, anamorphic Classicula R. Bauer, Begerow, Oberw. \& Marvanová 2003 (H)

Ustilaginaceae Tul. \& C. Tul.

Pseudozyma Bandoni emend. Boekhout 1985 (H)

Ustilaginomycetes, orders incertae sedis

Urocystidiales R. Bauer \& Oberw.

Urocystidaceae Begerow, R. Bauer, \& Oberw.

Paepalopsis J.G. Kühn 1882, anamorphic Urocystis Rabenh. ex Fuckel 1870

Paipalopsis J.G. Kühn 1882, anamorphic Urocystis Rabenh. ex Fuckel 1870 (H)

USTILAGINOMYCOTINA, order incertae sedis

Malasseziales R.T. Moore

Malassezia Baill. 1889

USTILAGINOMYCOTINA, genera incertae sedis

Botryoconis Syd. \& P. Syd. 1906 (H)

Tilletiella Zambett. 1970 (H)

BASIDIOMYCOTA, class incertae sedis Wallemiomycetes Zalar, de Hoog \& Schroers Wallemiales Zalar, de Hoog \& Schroers Wallemiaceae R.T. Moore
Wallemia Johan-Olsen 1887 (H)

BASIDIOMYCOTA, genera incertae sedis Anguillomyces Marvanová \& Bärl. 2000 (H)

Arcispora Marvanová \& Bärl. 1998 (H)

Bartheletica G. Arnaud, in Scheuer, R. Bauer, M. Lutz, Stabentheiner, Mel'nik \& Brube 2008 (H)

Gloeosynnema Seifert \& G. Okada 1988 (H)

Lactydina Subram. 1978('1977’) (H)

Microstella K. Ando \& Tubaki 1984 (H)

Nodulospora Marvanová \& Bärl. 2000 (H)

Pseudohelicomyces Garnica \& E. Valenz. 2000 (H)

Trichosporonoides Haskins \& J.F.T. Spencer 1967 (H)

Stachycoremium Seifert 1986 (H)

Stauriella Sivichai \& E.B.G. Jones 2004

(H)

\section{Discussion}

The study reveals that there are approximately 2895 anamorphic genera names of which 701 genera and 95 anamorph-like genera are linked to teleomorphic genera names, 447 are linked to teleomorph families, orders or classes, while for about 1728 (55\%) genera no teleomorph link is known.

The data used in this compilation are mostly from Index Fungorum, Species Fungorum, Kirk et al. (2008) and Seifert et al. (2011), plus publications during 2011 that were available before the manuscript was sent to press. Thus it is not inclusive and it is planned to improve the compilation in future issues. In future issues we will examine the literature from which connections are extracted to establish their basis.

Although we have used data from peer reviewed published data we have no way to confirm the validity of the species names used in various phylogenetic analyses. Where generic types were sequenced we have stated this, but again it is not clear whether correct names have been applied to these taxa or whether they are strains of type material (in most cases they are not).

The naming of fungi has long been problematic because of the dual nomenclature system. The system was introduced because it 
was previously often impossible to link a sexual form with the asexual form and therefore there was a need to be able to name both forms (Shenoy et al. 2007). With the advent of the molecular era it is often possible to link the morphs, and if there is no obvious teleomorph it is becoming increasing feasible to establish the relationship of the anamorphic genus within the teleomorph taxonomic framework (Wingfield et al 2011).

The following names were deleted from last years outline (Hyde et al. 2011) based on data in Seifert et al. (2011).

Abgliophragma R.Y. Roy \& Gujarati Acanthoderma Syd. \& P. Syd.

Aciculariella G. Arnaud

Acinula Fr.

Acrocylindrium Bonord

Acrospira Mont.

Acrosporium Bonord.

Acrotheciella Koord.

Acrothecium (Corda) Preuss

Actinochaete Ferro

Actinodochium Syd.

Actinonema Pers.

Actinospora Ingold

Agarwalia D.P. Tiwari \& P.D. Agrawal

Albophoma Tak. Kobay., Masuma, Omura

\& Kyoto Watan.

Aleurodomyces Buchner

Allescheriella Henn.

Allonema Syd.

Alysidium Kunze

Anthina Fr.

Arnaudina Trotter

Articularia Höhn.

Asterophora Ditmar

Asterophora Ditmar

Astrodochium Ellis \& Everh.

Attamyces Kreisel

Basididyma Cif.

Basramyces Abdullah, Abdulk. \& Goos

Bhargavaella Sarj. Singh \& K.S. Srivast.

Bilboque Viégas

Bilgramia Panwar, Purohit \& Chouhan

Bizzozeriella Speg.

Blastoconium Cif.

Blastostroma C.Z. Wei, Y. Harada \& Katum.

Botryodeorsum T.P. Devi, N. Mathur, Chowdhry, Jasvir Singh \& O. Prakash
Botryomyces Greco

Brachyhelicoon G. Arnaud

Broomeola Kuntze

Bulleribasidium J.P. Samp., M. Weiss \& R. Bauer

Bulleribasidium J.P. Samp., M. Weiss \& R. Bauer

Bulleromyces Boekhout \& Á. Fonseca

Bulleromyces Boekhout \& Á. Fonseca

Columnophora Bubák \& Vleugel

Cicadocola Brain

Discocolla Prill. \& Delacr.

Drechsleromyces Subram.

Dryadomyces Gebhardt

Eladia G. Sm. 1961

Graphilbum H.P. Upadhyay \& W.B. Kendr.

Helicoceras Linder

Helicosporina G. Arnaud ex Rambelli

Helminthophora Bonord.

Hennebertia M. Morelet

Heydenia Fresen.

Hormiactella Sacc.

Hormisciopsis Sumst.

Infrafungus Cif.

Jainesia Gonz. Frag. \& Cif.

Keratinomyces Vanbreus.

Loboa Cif., P.C. Azevedo, Campos \&

Carneiro 1956

Magdalaenaea G. Arnaud

Mammariopsis L.J. Hutchison \& J. Reid

Manoharachariomyces N.K. Rao, D.K.

Agarwal \& Kunwar

Mastigomyces Imshen. \& Kriss

Microides H.Y.Yip \& A.C. Rath

Mucobasispora Mustafa \& Abdul-Wahid

Multicladium K.B. Deshp. \& K.S. Deshp.

Multipatina Sawada

Myceloderma Ducomet

Mycobacillaria Naumov

Myropyxis Ces. ex Rabenh.

Naranus Ts. Watan.

Negeriella Henn.

Obstipispora R.C. Sinclair \& Morgan-Jones

Ochroconis de Hoog \& Arx

Ommatosporella Bat., J.L. Bezerra \& Poroca

Ordus K. Ando \& Tubaki

Panchanania Subram. \& N.G. Nair

Paradactylaria Subram. \& Sudha

Paradactylella Matsush.

Paraphialocephala Budathoki

Periola Fr.

Phragmodochium Höhn. 
Plectrothrix Shear

Pollaccia E.Bald. \& Cif.

Polyscytalina G. Arnaud

Polysynnema Constant. \& Seifert

Polythrinciopsis J. Walker

Poroisariopsis M. Morelet

Pseudocladosporium Braun. 1998

Pseudohansfordia G.R.W. Arnold

Pseudostilbella Munt.-Cvetk. \& GómezBolea

Pseudostracoderma A.E. Martinez \& Godeas

Pulvinotrichum Gamundí, Aramb. \& Giaiotti Racodium Pers.

Racodium Pers.

Ramalia Bat. 1957

Ramaraomyces N.K. Rao, Manohar. \& Goos

Ramoacrodictys G.Z. Zhao

Ramulaspera Lindr.

Rhabdomyces Balbiani

Roigiella R.F. Castañeda

Sagrahamala Subram.

Saliastrum myrtilli (Allesch.) Kujala

Septodochium Matsush.

Solheimia E.F. Morris 1967

Sphaerocolla P. Karst.

Sphaerocybe Magrou \& Marneffe

Stenocephalum Chamuris \& C.J.K. Wang

Stephanosporium Dal Vesco

Stevensomyces E.F. Morris \& Finley

Synnmukerjiomyces Aneja \& R. Kumar

Tretophragmia Subram. \& Natarajan

Tylomyces Cortini

Vamsapriya Gawas \& Bhat

Vasculomyces S.F. Ashby

Veronaella Subram. \& K.R.C. Reddy

Volutellis Clem. \& Shear

Volutellopsis Speg.

Yunnania H.Z. Kong

\section{Notes}

Notes are provided on recent published data on anamorphic taxa. Notes are provided if new data is published in relation to the phylogeny, if changes in anamorph or teleomorph linkages have been revealed and if new genera are introduced. If well known connections are confirmed we may provide data from one or more publications, however, in many cases we did not cite these data as the known connections are unchanged.
Acremonium Link

Summerbell et al. (2011) generated sequences for members of Acremonium and showed that within Hypocreales, there are two major clusters containing multiple Acremonium species. One clade contains A. sclerotigenum, the genus Emericellopsis, and the genus Geosmithia as prominent elements. The second clade contains the genera Gliomastix sensu stricto and Bionectria. In addition, there were numerous smaller clades plus two multi-species clades, one containing $A$. strictum and related species plus $A$. curvulum and related species. An account of Acremonium is provided in Seifert et al. (2011).

Acroconidiellina (Berk. \& Broome) M.B. Ellis Zhang et al. (2011a) stated that Acroconidiellina arecae is anamorphic Acantharia arecae.

\section{Acrostalagmus Corda}

Stilbella annulata was newly combined in Acrostalagmus annulatus (family Plectosphaerellaceae) by Reblova et al. (2011).

\section{Alternaria Nees}

Toth et al. (2011) described a new Alternaria species, as A. hungarica using macro and micro morphological examinations and ITS sequence analyses. Further, it was placed in Pleosporaceae. An account of Alternaria is provided in Seifert et al. (2011).

Antennariella Bat. \& Cif.

Chomnunti et al. (2011) suggested that the taxonomic placement is uncertain.

Aquaphila Goh, K.D. Hyde \& W.H. Ho

Boonmee et al. (2011) confirmed this genus as anamorphic Tubeufia.

Aspergillus P. Micheli ex Link

Sexual reproduction was examined in Aspergillus nomius by Horn et al. (2011). Crosses between sexually compatible strains resulted in the formation of multiple nonostiolate ascocarps within stromata, which places the teleomorph in genus Petromyces in Trichocomaceae. Ascocarp and ascospore morphology in $P$. nomius were similar to that in $P$. flavus and $P$. parasiticus, and difference 
between telemorphs were insufficient for species separation. An account of Aspergillus is provided in Seifert et al. (2011).

\section{Beauveria Vuill.}

Rehner et al. (2011) carried out multilocus phylogeny of Beauveria based on partial sequences of $R P B 1, R P B 2, T E F$ and the nuclear intergenic region, Bloc. They assessed diversity within the genus and evaluated species concepts and their taxonomic status. Further, they proposed types for $B$. bassiana and $B$. brongniartii while describing six new species. An account of Beauvaria is provided in Seifert et al. (2011).

Chalara (Corda) Rabenh.

Seifert et al. (2011) reported four teleomorph species and tentatively another twelve species that can be teleomorphs.

\section{Ciferrioxyphium Bat. \& H. Maia}

The teleomorph, Aithaloderma was transferred to Chaetothyriaceae by Chomnunti et al. (2011)

\section{Cladobotryum Nees}

Poldmaa (2011) reported twelve new species of Cladobotryum/Hypomyces in Hypocreaceae. Ten of these were described as new species, including teleomorphs for two previously known anamorphic species; Cladobotryum coriolopsicola anamorphic Hypomyces samuelsii and Cladobotryum virescens anamorphic Hypomyces virescens. Also Cladobotryum heterosporum, $C$. indoafrum, and $C$. tchimbelense were described as new anamorphic species.

\section{Conidiocarpus Woron.}

Chomnunti et al. (2011) confirmed this genus to be anamorphic Phragmocapnias.

\section{Custingophora Stolk, Hennebert \& Klopotek}

Species of Gondwanamyces and their Custingophora anamorphs were introduced into a new family Gondwanamycetaceae, by Reblova et al. (2011a).

\section{Cylindrocarpon Wollenw.}

Chaverri et al. (2011) showed five distinct highly supported clades that correspond to some extent with the informal Cylindrocarpon (anamorph) and Neonectria (telomorph) groups and three of which are newly described. The following genera were recognised: (1) Neonectria / Cylindrocarpon sensu stricto (N. coccinea-group); (2) N. rugulosagroup, hereafter Rugonectria gen. nov.; (3) $N$. mammoidea / veuillotiana-group, hereafter Thelonectria gen. nov.; (4) N. radicicola group, hereafter Ilyonectria gen. nov.; and (5) Campylocarpon. and also, results showed that Neonectria/Cylindrocarpon sensu stricto clusters with Neonectria fuckeliana, and $T$. jungneri with Thelonectria.

Cylindrotrichum Bonord.

Reblova et al. (2011b) introduced a new genus Reticulascus for Chaetosphaeria tulasneorum with associated Cylindrotrichum anamorph and placed in new family Reticulascaceae.

\section{Discosia Lib.}

Tanka et al. (2011) showed Discosia was not monophyletic and was separated into two distinct lineages. The new genus Immersidiscosia (family Amphisphaeriaceae) was erected on the basis of SSU, LSU and ITS nrDNA and $\beta$-tubulin gene sequences. Further, they stated this genus anamorphic Adisciso.

\section{Eriothyrium Speg.}

$\mathrm{Wu}$ et al. (2011) transferred the teleomorp to Asterinaceae and confirmed to be anamorphic Asterinema

Fumiglobus D.R. Reynolds \& G.S. Gilbert

Chomnunti et al. (2011) listed the placement of this genus in Capnodiaceae as doubtful.

\section{Fusarium Link}

Schroers et al. (2011) reinstated the connection of of Fusarium buxicola with the bluish black and $\mathrm{KOH}+$ perithecial species Cyanonectria buxi. The connection of $F$. buxicola is often erroneously reported as the anamorph of Geejayessia desmazier.

A new species, Fusarium musae was described together with its teleomorph Gibberella musae by Hove et al. (2011).

Scauflaire et al (2011) used a robust 
polyphasic approach to describe a new biological species, Fusarium temperatum, within the Gibberella fujikuroi species complex.

\section{Geosmithia Pitt}

Geosmithia morbida is a new species described by the Kolarik et al. (2011) and this is the first report of a phytopathogenic species in this genus and in the Bionectriaceae. $G$. morbida has been isolated only from Pityophthorus juglandis or from necrotic phloem associated with $P$. juglandis feeding on Juglans species in western USA. The telemorph is unknown.

\section{Gliomastix Guég.}

Gliomastix (Hypocreales) was revived for five species, G. murorum, G. polychroma, $G$. tumulicola, G. roseogrisea, and G. masseei by Summerbell et al. (2011)

Heliocephala V.G. Rao, K.A. Reddy \& de Hoog

Abarca et al. (2011) recognized Heliocephala and Holubovaniella as synonymous and described a new species Heliocephala triseptata. All the species were positioned in Dothideomycetes and identified Stomiopeltis betulae (Micropeltidaceae) its closest relative.

\section{Helicoma Corda}

Boonmee et al. (2011) confirmed this genus to be anamorphic Tubeufia and Thaxteriella Further they introduced a new genus Chlamydotubeufia and $H$. Chlamydosporum and $H$. depressispora were transered to a new genus.

\section{Helicoön Morgan}

Boonmee et al. (2011) confirmed this genus to be anamorphic Tubeufiaceae.

\section{Helicomyces Link}

Boonmee et al. (2011) ensured that Helicomyces to be anamorphic Acanthostigma.

\section{Helicosporium Nees}

Boonmee et al. (2011) confirmed that Helicosporium to be anamorphic Acanthostigma and Tubeufia.

\section{Holubovaniella R.F. Castañeda}

Abarca et al. (2011) placed all the species in Dothideomycetes and identified Stomiopeltis betulae (Micropeltidaceae) as their closest relative by analysing large subunit rDNA.

\section{Hyphopodia-like}

Suetrong et al. (2011) introduced a new family, Trematosphaeriaceae. Trematosphaeria is the type genus and Hyphopodia-like anamorphs were recorded.

Immersidiscosia Kaz. Tanaka, Okane \& Hosoya

Discosia eucalypti deviated from Discosia clade and was transferred to a new genus, Immersidiscosia (family Amphisphaeriaceae) by Tanaka et al. (2011) on the basis of SSU, LSU and ITS nrDNA and $\beta$-tubulin gene sequencing.

\section{Leptographium Lagerb. \& Melin}

Kim et al. (2011) recorded a new Leptographium species, L. tereforme (Ophiostomataceae) isolated from the bark beetle Hulurgus ligniperda.

\section{Leptoxyphium Speg.}

The teleomorph, Aithaloderma was transferred to Chaetothyriaceae by Chomnunti et al. (2011).

\section{Lomaantha Subram.}

Ma et al. (2011) described a new species Lomaantha phragmitis (anamorphic Pezizomycotina) and provided a key and synoptic table of morphological characters of all three Lomaantha species.

Lolia Abdel- Aziz \& Abdel- Wahab

Phylogenetic analyses of the $28 \mathrm{~S}$ ribosomal large subunit (LSU) rDNA sequence placed the new fungus in the family Lindgomycetaceae, Pleosporales.

Mariannaea G. Arnaud ex Samson

A new species of Mariannaea (family Nectriaceae) was described as $M$. samuelsii by Grafenhan et al. (2011). 
Microcera Desm.

Grafenhan et al. (2011) described a new species as Microcera rubra.

Mirandina G. Arnaud ex Matsush.

Boonmee et al. (2011) removed the teleomorph Taphrophila from Tubeufiaceae.

Monilochaetes Halst. ex Harter

Reblova et al. (2011) confirmed the synonymy of Monilochaetes with Dischloridium.

Microxiphium (Harv. ex Berk. \& Desm.) Thüm.

Chomnunti et al. (2011) stated that this is polyphyletic and included Coccodiniaceae.

Phaeoxyphiella Bat. \& Cif. 1963

Chomnunti et al. (2011) confirmed Phaeoxyphiella to be anamorphic Capnodium.

Polychaeton (Pers.) Lev.

Chomnunti et al. (2011) confirmed this genus to be anamorphic Capnodiaceae.

\section{Polychaetella Speg.}

Chomnunti et al. (2011) stated that the status of this genus in Capnodiaceae is uncertain.

Pseudotripoconidium Z.F. Yu, Ying Zhang \& K.Q. Zhang

A new anamorph genus Pseudotripoconidium was proposed by the $\mathrm{Yu}$ et al. (2011) using morphological and molecular analyses. It was described based on four isolates from ascospores of Orbilia aff. luteorubella. Also, this fungus differs from previously known Orbilia anamorphs in producing inversely pyramidal, unicellular conidia with several protuberances at their distal end.

Repetophragma Subram.

Ruiz et al. (2011) described a new species $R$. paracambrense from Mexico by the morphology of its conidia.

Rhynchosporium Heinsen ex A.B. Frank

Two new species of Rhynchosporium anamorphic Dermateaceae ( $R$. commune and
$R$. agropyri) were described by the Zaffarano et al. (2011).

\section{Sarcinella Sacc.}

A new species of Sarcinella ( $S$. tamarindi) and its Questieriella synanamorph (family Englerulaceae) were reported by Hosagoudar \& Riju (2011).

Scolecoxyphium Cif. \& Bat.

Chomnunti et al. (2011) confirmed this genus to be anamorphic Capnodium and Scorias.

\section{Stachylidium Link}

Stachylidium was added in to the family Plectosphaerellaceae by Reblova et al. (2011)

Sterigmatobotrys Oudem.

Reblova \& Seifert (2011) reported the teleomorph of Sterigmatobotrys macrocarpa (anamorphic Pezizomycotina) to be morphologically similar to the species of genera Carpoligna and Chaetosphaeria; but ncLSU phylogeny confirmed that Sterigmatobotrys is closely related to Carpoligna and its Pleurothecium anamorph and to the anamorphic species Pleurothecium obovoideum.

\section{Stilbella Lindau}

Jaklitsch \& Voglmayr (2011) described a new telemorph Nectria eustromatica which has an anamorph, shares morphological traits with the genera Stilbella but produces nonphialidic macroconidia in addition to phialoconidia.

\section{Sympodiella-like}

Zhang et al. (2011a) reported Symodiella-like anamorphs from Sympoventuria.

\section{Trichoderma Pers.}

A new species of Trichoderma (anamorphic Hypocrea) (family Hypocreaceae), T. amazonicum, endophytic on the living sapwood and leaves of Hevea spp. trees was described by Chaverri et al. (2011).

\section{Trichothecium Link}

The genus Trichothecium was revised following the principles of unitary nomenclature based on the oldest valid anamorph 
or teleomorph name, and new combinations are made in Trichothecium for the tightly interrelated Acremonium crotocinigenum, Spicellum roseum, and teleomorph Leucosphaerina indica by Summerbell et al. (2011).

\section{Tripospermum Speg.}

Seifert et al. (2011) stated that Tripospermum to be anamorphic Triposporiopsis. However Chomnunti et al. (2011) suggested its status is uncetian.

\section{Tubercularia Weber ex F.H. Wigg}

Jaklitsch \& Voglmayr (2011) described a new telemorph Nectria eustromatica with an anamorph that shares morphological traits with Tubercularia but produces non-phialidic macroconidia in addition to phialoconidia.

Veronaeopsis Arzanlou \& Crous

Zhang et al. (2011a) transferred this genus to a new family Sympoventuriaceae.

Zymoseptoria Quaedvlieg \& Crous

Quaedvlieg et al. (2011) described the new genus Zymoseptoria (anamorphic Mycosphaerella).

\section{References}

Abarca GH, Ruiz RFC, Mota RMA, Hernandez CIB, Gomez S, Bogale M, Untereiner WA. 2011 - A new species of Heliocephala from Mexico with an assessment of the systematic position of the anamorph genera Heliocephala and Holubovaniella. Mycologia 103, 631640.

Abdel-Aziz, Faten A, Mohamed A. AbdelWahab. 2010 - Lolia aquatica gen. et sp. nov. (Lindgomycetaceae, Pleosporales), a new coelomycete from freshwater habitats in Egypt. Mycotaxon $114,33-42$.

Boonmee S, Zhang Y, Chomnunti P, Chukeatirote E, Tsui CKM, Bahkali AH, Hyde KD. 2011 - Revision of lignicolous Tubeufiaceae based on morphological reexamination and phylogenetic analysis. Fungal Diversity 51, 63-102.

Chaverri P, Gazis RO, Samuels GJ. 2011 -
Trichoderma amazonicum, a new endophytic species on Hevea brasiliensis and $H$. guianensis from the Amazon basin. Mycologia 103, 139-151.

Chaverri P, Salgado C, Hirooka Y, Rossman AY, Samuels GJ. 2011 - Delimitation of Neonectria and Cylindrocarpon (Nectriaceae, Hypocreales, Ascomycota) and related genera with Cylindrocarpon-like anamorphs. Studies in Mycology 68, 57-78.

Chomnunti P, Schoch CL, Aguirre-Hudson B, KoKo TW, Hongsanan S, Jones EBG, Kodsueb R, Phookamsak R, Chukeatirote E, Bahkali AH, Hyde KD. 2011 - Capnodiaceae. Fungal Diversity 51, 103-134.

Crous PW, Tanka K, Summerell BA, Groenewald JZ. 2011 - Additions to the Mycosphaerella complex. IMA Fungus 2, 49-64.

Grafenhan T, Schroers HJ, Nirenberg HI, Seifert KA. 2011 - An overview of the taxonomy, phylogeny, and typification of nectriaceous fungi in Cosmospora, Acremonium, Fusarium, Stilbella, and Volutella. Studies in Mycology 68, 79113.

Hawksworth DL, Crous PW, Redhead SA, Reynolds Wingfeld MJ, et al 2011 The Amsterdam Declaration on Fungal Nomenclature. IMA Fungus 2, 105112; Mycotaxon 116, 491-500.

Hawksworth DL. 2011 - Naming Aspergillus species: progress towards one name for each species. Medical Mycology 49(Suppl. 1), S70-S76.

Horn BW, Moore GG, Carbone I. 2011 Sexual reproduction in aflatoxinproducing Aspergillus nomius. Mycologia 103, 174-183.

Hosagoudar VB, Riju MC. 2011 - Sarcinella tamarindi sp. nov. from Kerala, India. Mycosphere 2, 157-160.

Hove VF, Waalwijk C, Logrieco A, Munaut F, Moretti A. 2011 - Gibberella musae (Fusarium musae) sp. nov., a recently discovered species from banana is sister to $F$. verticillioides. Mycologia 103, 570-585.

Hyde KD, McKenzie EHC, KoKo TW. 2011 Towards incorporating anamorphic 
fungi in a natural classification checklist and notes for 2010. Mycosphere 2, 1-88.

Jaklitsch WM, Voglmayr H. 2011 - Nectria eustromatica sp. nov., an exceptional species with a hypocreaceous stroma. Mycologia 103, 209-218.

Kim S, Harrington TC, Lee JC, Seybold SJ. 2011 - Leptographium tereforme sp. nov. and other Ophistomatales isolated from the root-feeding bark beetle Hylurgus ligniperda in California. Mycologia 103, 152-163.

Kirk PM, Cannon PF, Minter DW, Stalpers JA. 2008 - Dictionary of the fungi, $10^{\text {th }}$ edn. CABI publishing, Wallingford, UK.

Kolarik M, Freeland E, Utley C, Tisserat N. 2011 - Geosmithia morbida sp. nov., a new phytopathogenic species living in symbiosis with the walnut twig beetle (Pityophthorus juglandis) on Juglans in USA. Mycologia 103, 325-332.

Lumbsch HT, Huhndorf SM. 2010 - Outline of Ascomycota-2009; Notes on ascomycete systematics. Nos. 4751-5113. Myconet 14, 1-69.

Ma J, Wang Y, O’Neill N, Zhang XG. 2011 A revision of the genus Lomaantha, with the description of a new species. Mycologia 103, 407-410.

Nag Raj TR. 1993. Coelomycetous anamorphs with appendage-bearing conidia. Mycologue Publications, Waterloo, Canada.

Poldmaa K. 2011 - Tropical species of Cladobotryum and Hypomyces producing red pigments. Studies in Mycology 68, 1-34.

Quaedvlieg W, Kema GHJ, Groenewald JZ, Verkley GJM, Seifbarghi S, Razavi M, Mirzadi Gohari A, Mehrabi R, Crous PW. 2011 - Zymoseptoria gen. nov.: a new genus to accommodate Septorialike species occurring on graminicolous hosts. Persoonia 26, 57-69.

Reblova M, Seifert KA. 2011a - Discovery of the teleomorph of the hyphomycete, Sterigmatobotrys macrocarpa, and epitypification of the genus to holomorphic status. Studies in Mycology 68, 193-202.
Reblova M, Gams W, Seifert KA. 2011b Monilochaetes and allied genera of the Glomerellales, and a reconsideration of families in the Microascales. Studies in Mycology 68, 163-191.

Rehner SA, Minnis AM, Sung GH, Luangsaard JJ, Devotto L, Humber RA. 2011 Phylogeny and systematics of the anamorphic, entomopathogenic genus Beauveria. Mycologia 103, 1055-1073

Samuels GJ, Ismaiel A. 2011 - Hypocrea peltata: a mycological Dr Jekyll and $\mathrm{Mr}$ Hyde?. Mycologia 103, 616-630.

Scauflaire J, Gourgue M, Munaut F. 2011 Fusarium temperatum sp. nov. from maize, an emergent species closely related to Fusarium subglutinans. Mycologia 103, 586-597.

Schroers HJ, Grafenhan T, Nirenberg HI, Seifert KA. 2011 - A revision of Cyanonectria and Geejayessia gen. nov., and related species with Fusarium-like anamorphs. Studies in Mycology 68, 115-138.

Seifert KA, Gams W. 2011 The genera of Hyphomycetes - update. Persoonia 27, $119-12$.

Seifert K, Morgan-Jones G, Gams W, Kendrick B. 2011 - The Genera of Hyphomycetes CBS-KNAW Fungal Biodiversity Centre Utrecht, The Netherlands.

Suetrongs S, Hyde KD, Zhang Y, Bahkali AH, Jone EBG. 2011 - Trematosphaeriaceae fam. nov. (Dothideomycetes, Ascomycota) Cryptogamie Mycologie 32, $1-16$.

Summerbell RC, Gueidan C, Schroers HJ, Hoog GSD, Starink M, Rosete YA, Guarro J, Scott JA. 2011 - Acremonium phylogenetic overview and revision of Gliomastix, Sarocladium and Trichotecium. Studies in Mycology 68, 139162.

Sutton BC. 1980 - The Coelomycetes- Fungi imperfecti with Pycnidia, Acervuli and Stromata. Commonwealth Mycological Institute, Kew, UK.

Tanaka K Endo M, Hirayama K, Okane I, Hosoya T, Sato T. 2011 - Phylogeny of Discosia and Seimatosporium, and introduction of Adisciso and Immersidi- 
scosia genera nova. Persoonia 26, 8598.

Taylor JW. 2011 - One Fungus = One Name: DNA and fungal nomenclature twenty years after PCR. IMA Fungus 2, 113120.

Toth B, Csosz M, Szabo-Hever A, Simmons EG, Samson RA, Varga J. 2011 Alternaria hungarica sp. nov., a minor foliar pathogen of wheat in Hungary. Mycologia 103, 94-100.

Wang Y, Geng Y, Ma J Wang Q, Zhang XG. 2011 - Sinomyces: a new genus of anamorphic Pleosporaceae. Fungal Biology 115, 188-195.

Wingfield MJ, de Beer ZW, Slippers B, Wingfield BD, Groenewald JZ, Lombard L, Crous PW. 2011 - One fungus one name promotes progressive plant pathology. Molecular Plant Pathology : in press
Wu HX, Schoch CL, Boonmee S, Bahkali AH Chomnunti P, Hyde KD. 2011 - A reappraisal of Microthyriaceae. Fungal Diversity 51, 189-248.

Yu ZF Qiao M, Zhang Y Qin L, Zhang KQ. 2011 - Pseudotripoconidium, a new anamorph genus connected to Orbilia. Mycologia 103, 164-173.

Zaffarano PL, McDonald BA, Linde CC. 2011 - Two new species of Rhynchosporium. Mycologia 103, 195-202.

Zhang Y, Crous PW, Schoch CL, Bahkali AH, Guo LD, Hyde KD 2011a - A molecular, morphological and ecological re-appraisal of Venturiales a new order of Dothideomycetes. Fungal Diversity 51, 249-277.

Zhang Y, Koko TW, Hyde KD. 2011b Towards a monograph of Dothideomycetes: studies on Diademaceae Cryptogamie Mycologiae 32, 115-126. 